Check for updates

Cite this: Chem. Sci., 2019, 10, 6035

๑ All publication charges for this article have been paid for by the Royal Society of Chemistry

Received 4th April 2019

Accepted 23rd May 2019

DOI: 10.1039/c9sc01652j

rsc.li/chemical-science

\section{Fluorescent probes for organelle-targeted bioactive species imaging}

\author{
Peng Gao, (D) Wei Pan, (D) Na Li (D) * and Bo Tang (D) *
}

Bioactive species, including reactive oxygen species (ROS, including $\mathrm{O}_{2}{ }^{\cdot-}, \mathrm{H}_{2} \mathrm{O}_{2}, \mathrm{HOCl},{ }^{1} \mathrm{O}_{2},{ }^{\circ} \mathrm{OH}, \mathrm{HOBr}$, etc.), reactive nitrogen species (RNS, including $\mathrm{ONOO}^{-}, \mathrm{NO}, \mathrm{NO}_{2}, \mathrm{HNO}$, etc.), reactive sulfur species (RSS, including GSH, Hcy, Cys, $\mathrm{H}_{2} \mathrm{~S}, \mathrm{H}_{2} \mathrm{~S}_{n}, \mathrm{SO}_{2}$ derivatives, etc.), ATP, $\mathrm{HCHO}, \mathrm{CO}$ and so on, are a highly important category of molecules in living cells. The dynamic fluctuations of these molecules in subcellular microenvironments determine cellular homeostasis, signal conduction, immunity and metabolism. However, their abnormal expressions can cause disorders which are associated with diverse major diseases. Monitoring bioactive molecules in subcellular structures is therefore critical for bioanalysis and related drug discovery. With the emergence of organelle-targeted fluorescent probes, significant progress has been made in subcellular imaging. Among the developed subcellular localization fluorescent tools, ROS, RNS and RSS (RONSS) probes are highly attractive, owing to their potential for revealing the physiological and pathological functions of these highly reactive, interactive and interconvertible molecules during diverse biological events, which are rather significant for advancing our understanding of different life phenomena and exploring new technologies for life regulation. This review mainly illustrates the design principles, detection mechanisms, current challenges, and potential future directions of organelle-targeted fluorescent probes toward RONSS.

\section{Introduction}

Revolutions in science and technology have greatly enhanced our lives. As a prominent example, in the past few decades fluorescent imaging technology has made great achievements, which have brought increasingly fascinating news for diverse fields including biomedicine and bioanalysis. ${ }^{1}$ Extensive evidence shows that diverse bioactive species are critical for conducting physiological processes throughout the cell cycle, and numerous major diseases are associated with the abnormal expression of these species. ${ }^{2}$ Meanwhile, the concentrations of these molecules are inhomogeneous in different subcellular environments at different times. ${ }^{3}$ Therefore, a better understanding of diverse physiological and pathological processes can be obtained via accurate monitoring of subcellular molecular concentrations of these molecules, which is beneficial for exploring novel strategies for regulating biological phenomena and improving diagnosis, treatment and prognosis of different diseases. ${ }^{4}$

Subcellular microenvironments are maintained by the cell endomembrane system, typically known as organelles, including the nucleus, mitochondria, lysosomes, endoplasmic reticulum (ER), Golgi apparatus, lipid droplets and so on. These

College of Chemistry, Chemical Engineering and Materials Science, Collaborative Innovation Center of Functionalized Probes for Chemical Imaging in Universities of Shandong, Key Laboratory of Molecular and Nano Probes, Ministry of Education, Institute of Molecular and Nano Science, Shandong Normal University, Jinan 250014, P. R. China. E-mail: lina@sdnu.edu.cn; tangb@sdnu.edu.cn organelles with complex but ordered structures play important roles in ensuring the smooth progress of cell growth, proliferation, fission, signal transduction and apoptosis. They are also the main sources as well as targets of diverse bioactive species. However, the disruption of intracellular homeostasis can result in various disorders. ${ }^{2 g, 3 a, c, d}$ For example, owing to the extremely rapid respiration rate, reactive oxygen species (ROS) are usually overexpressed in diverse organelles of cancer cells, which are also associated with lower intracellular $\mathrm{pH}$. To maintain cell proliferation, the contents of antioxidants in malignant cells are elevated accordingly. Therefore, detecting the real status of subcellular microenvironments is critical in understanding different pathogeneses of a broad spectrum of major diseases.

Unfortunately, the real-time, in situ, dynamic, accurate, and reversible monitoring of bioactive molecules in subcellular microenvironments has been a great challenge due to theoretical and technological limitations, and many related investigations have remained at the cellular level during the last few decades. ${ }^{1 \boldsymbol{e}, \boldsymbol{g}, \boldsymbol{3} \boldsymbol{c}, \boldsymbol{d , 5}}$ Notably, over the past several years, significant efforts have been devoted to the design and development of organelle-targeted fluorescent probes for precise monitoring of subcellular microenvironments, including $\mathrm{pH},{ }^{6}$ polarity, ${ }^{7}$ ions, ${ }^{8}$ viscosity, ${ }^{9}$ ROS (including $\mathrm{O}_{2}{ }^{\cdot-}, \mathrm{H}_{2} \mathrm{O}_{2}, \mathrm{HOCl},{ }^{1} \mathrm{O}_{2},{ }^{\circ} \mathrm{OH}, \mathrm{HOBr}$, etc.), ${ }^{10}$ reactive nitrogen species (RNS, including $\mathrm{ONOO}^{-}, \mathrm{NO}$, $\mathrm{NO}_{2}, \mathrm{HNO}$, etc.), ${ }^{\mathbf{1 1}}$ reactive sulfur species (RSS, including $\mathrm{GSH}$, Hcy, Cys, $\mathrm{H}_{2} \mathrm{~S}, \mathrm{H}_{2} \mathrm{~S}_{n}, \mathrm{SO}_{2}$ derivatives, etc.), ${ }^{1 \boldsymbol{e}_{f}, \mathbf{1 2}} \mathrm{ATP},{ }^{13} \mathrm{HCHO}^{14}$ and so on. Among these targets, ROS, RNS and RSS (termed 
RONSS) have attracted increasing interest because they are closely related to the intracellular redox homeostasis. In other words, they are capable of buffering the subcellular redox balance during different processes, which can further determine a number of biological events. Therefore, organelletargeted RONSS fluorescent probes are of great significance for explaining and exploring the physiological and pathological functions of these molecules, and could offer theoretical bases for clinical diagnosis as well as drug design. ${ }^{2 g, 3 b-\boldsymbol{d}, \mathbf{4 c}}$

This review mainly focuses on the latest achievements of organelle-targeted fluorescent probes for RONSS imaging. The design principles, detection mechanisms and biological applications of these fluorescence tools are carefully illustrated. Moreover, the current challenges and the potential future directions, which would evoke more research interest and open new avenues for biological analysis, are included as well.

\section{General rules for functional probe design}

The advancement of microscopic imaging technology has introduced new opportunities for visualizing subtle intracellular structures. To investigate the critical events in living cells on the organelle and even more subtle levels, elaborately designed fluorescent probes are indispensable. ${ }^{15}$ Currently, benefiting from their easy design, preparation and functionalization, fluorescent molecular and nanoprobes are being widely employed for biological imaging. ${ }^{1 \boldsymbol{a}, \boldsymbol{b}, \boldsymbol{f}, \mathbf{2 c}}$ Organelle-targeted fluorescent probes mainly contain three moieties: targeting groups, fluorophores, and recognition units. ${ }^{3 a, d}$ Benefiting from the different physiochemical properties of diverse organelles, probes with diverse targeting groups can localize in specific organelles. $^{3 b}$ After reaching subcellular locations of interest, they can subsequently react with diverse bioactive species through specific reaction mechanisms and further make detectable signal changes via different response mechanisms, which enables us to readily and effectively monitor these species in different organelles. In this part, diverse targeting approaches, reaction mechanisms and response mechanisms of organelle-targeted RONSS probes will be elaborated.

\subsection{Approaches for delivering fluorescent probes into organelles}

2.1.1. Nucleus-targeting. As the container of the majority of DNA in cells, the nucleus is the most important organelle of eukaryotic cells. ${ }^{16}$ Detection of RONSS in the nucleus of living cells holds great promise for chemical biology. For instance, Chang's group ${ }^{\mathbf{1 7}}$ reported a nucleus-targeted $\mathrm{H}_{2} \mathrm{O}_{2}$ probe (NucPE1) and successfully revealed the reduced nuclear $\mathrm{H}_{2} \mathrm{O}_{2}$ level in sir-2.1 over-expressed worms, which revealed the relationships between enhanced ROS regulation capability and longevity (see details in Section 3.1).

Typically, the nucleus is enveloped by a double-layered membrane, which contains numerous nuclear pores as well as ribosomes. Some small molecules and ions can permeate through the nuclear membrane freely, while large biomolecules, such as ribosomes and RNA, can pass through the nuclear pores via energy related pathways. By targeting the minor grooves in negatively charged DNA double-strands, small cationic fluorescent probes with two or more cationic centers and hydrophobic planar aromatic structures can preferentially label DNA molecules, and some of them have been commercialized (Fig. 1A). ${ }^{\mathbf{1 6 a}, \mathbf{1 8}}$ In addition to labelling DNA, decorating probes with nuclear localization signal peptides, which enables binding of probes with importins and further actively delivering them into the nucleus through nuclear pores, is also an established protocol. ${ }^{16 b, 19}$

2.1.2. Mitochondrion-targeting. Mitochondria are doublemembrane constructed organelles. They are the main compartments for intracellular respiration (oxidative phosphorylation). ${ }^{4 a}$ Mitochondria regulate energy generation, calcium circulation, protein synthesis, apoptosis pathways and so on. It is well known that mitochondria are the primary sources of diverse ROS and RNS. Therefore, probes that specifically accumulate in mitochondria play essential roles in monitoring the mitochondrial function and investigating numerous mitochondrion-related diseases. ${ }^{\mathbf{9 a , 2 0}}$

During mitochondrial respiration, the proton pumps in the inner mitochondrial membrane transport protons to the mitochondrial intermembrane space, which results in the formation of a highly negative mitochondrial transmembrane potential (MMP, $\sim-180 \mathrm{mV}$ ). ${ }^{21}$ Inspired by this process, many delocalized lipophilic cations have been proved to exhibit mitochondrion targeting capabilities. ${ }^{22}$ Fluorescent probes with intrinsic or post-functionalized cationic aromatic structures can be used for mitochondrial imaging. Typical targeting ligands including triphenylphosphonium (TPP), positively charged pyridine and quinoline derivatives, cyanine and rhodamine are popular fluorophores for designing mitochondrion-targeted probes (Fig. 1B). ${ }^{3 \boldsymbol{d}, 7 \boldsymbol{a}, 23}$ In addition to MMP, mitochondrial transport proteins have also been used as targets. Some functional groups, such as peptides and pyruvate, have also been developed for constructing mitochondrion-targeted probes owing to their affinities for specific mitochondrial proteins. ${ }^{24}$

2.1.3. Lysosome-targeting. Lysosomes are the digestive compartments of cells. Acidic microenvironments $(\mathrm{pH} \sim 5.0)$ and an abundance of hydrolases are the most prominent properties of lysosomes with a single-layered membrane structure. Lysosomes play essential roles in living cells. They take charge of foreign substance scavenging, digestion and autophagy. ${ }^{25}$ RONSS are capable of regulating the lysosomal enzyme activity and membrane permeability, which can further affect diverse biological processes. Thus, monitoring RONSS in lysosomes has become another important task for chemical biology.

Currently, modification of probes with lipophilic amines is the most widely employed strategy for driving probes into lysosomes/endosomes. ${ }^{\mathbf{6}, \mathbf{2 6}}$ The protonated amines in lysosomes are membrane impermeable, which results in the lysosomal/ endosomal selective entrapment of the probes. 4-(2-Hydroxyethyl) morpholine and $N, N$-dimethylethylenediamine are the most popular groups for constructing lysosome-targeted fluorescent probes (Fig. 1C) ${ }^{26 a, b, 27}$ Interestingly, fluorescent probes without lysosomotropic groups can also be used to detect 
A<smiles>[R]c1ccc(C2Nc3ccc(-c4nc5cc(N6CC[NH+](C)CC6)ccc5[nH]4)cc3N2)cc1</smiles>

Hoechst $33258 \mathrm{R}=-\mathrm{OH}$

Hoechst $33342 \mathrm{R}=-\mathrm{CH}_{2} \mathrm{CH}_{3}$

Hoechst $34580 \mathrm{R}=-\mathrm{N}\left(\mathrm{CH}_{3}\right)_{2}$

B
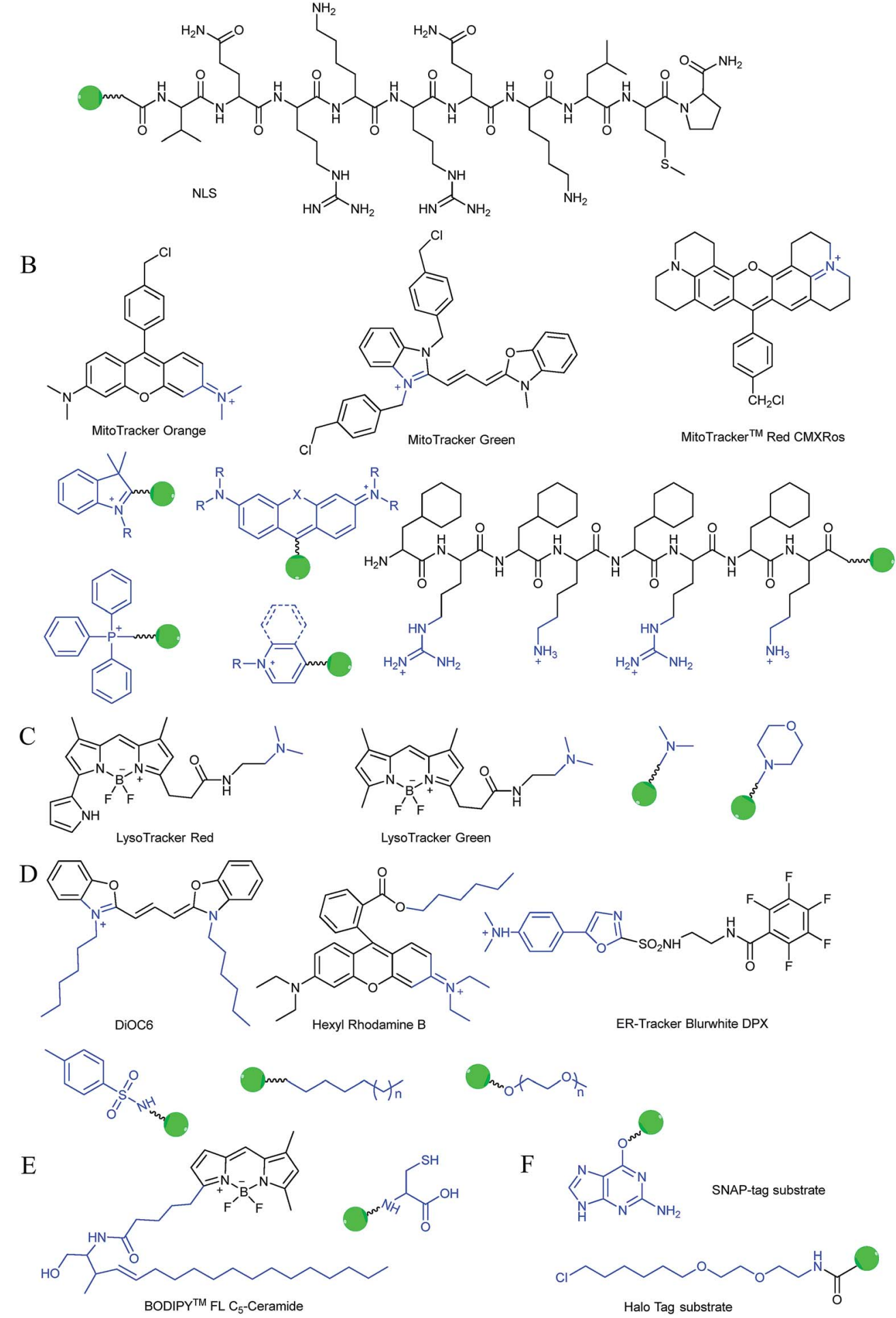<smiles>N=C(N)c1ccc(-c2nc3ccc(C(=N)N)cc3[nH]2)cc1</smiles>

DAPI

Fig. 1 Structures of diverse commercialized organelle-labelling dyes and common groups for organelle-targeted probe design: (A) for nucleus, (B) for mitochondria, (C) for lysosomes, (D) for endoplasmic reticulum, (E) for Golgi apparatus and (F) substrates for protein tagging probes.

species in lysosomes specifically, and they are usually logic molecules and can only release fluorescence signals in acidic environments after reacting with analytes. ${ }^{28}$
2.1.4. Endoplasmic reticulum/Golgi apparatus-targeting. The ER can be divided into rough ER and smooth ER based on whether they contain ribosomes. Typically, the rough ER takes 
charge of protein synthesis, while the smooth ER mainly controls lipid and carbohydrate metabolism, calcium signalling and so on. The intrinsic molecular mechanism of ER-targeted fluorescence probes remains unclear, but the common features of these probes may be concluded as small amphiphilic or lipophilic cationic molecules, and some ER specific dyes have been commercialized (Fig. 1D). ${ }^{29}$ Recently, by using $p$-toluene sulfonamide as the ER localization group, our group developed several fluorescent probes for ER-targeted imaging. ${ }^{7 \boldsymbol{b}, \boldsymbol{d}, \mathbf{3 0}}$

The Golgi apparatus is another important intracellular compartment for the modification, storage and transportation of lipids, carbohydrates and protein. After modification and labelling, cargoes in the Golgi apparatus can be transferred into their final destinations, such as lysosomes and the cytoplasmic membrane, and subsequently exert their biological functions. It has been proved that a stress-signalling overload in the Golgi apparatus can induce a series of disorders, which can further cause diverse diseases as well as cell death. Therefore, development of efficient Golgi apparatus specific probes has also attracted certain interest in recent years. ${ }^{3 b, 31}$ However, the intrinsic rules of Golgi apparatus-targeted probes are still vague, which has severely hampered the development of related fluorescent tools. Very recently, inspired by the rich cysteine residues in Golgi apparatus localized galactosyltransferase and protein kinase D, Huang and co-workers demonstrated $\mathrm{L}^{-}$ cysteine (Fig. 1E) as an efficient Golgi apparatus targeting ligand, and several probes have been developed using this strategy. ${ }^{31 a-c}$

2.1.5. Protein tagging. In addition to the above targeting strategies, protein tagging is another widely investigated method for anchoring probes into organelles. The general procedure for protein tagging is to transfect and express specific fusion tags/enzymes with the protein of interest (POI). Subsequently, probes containing related substrates can spontaneously and efficiently connect with tags via highly specific biochemical reactions. By choosing different plasmids, fusion tags can bind with specific protein in different locations, thereby enabling probes to be labelled into different organelles. The major advantage of the protein tagging technique is the covalent connection of probes with protein, which is more stable, less toxic and environmentally insensitive. Importantly, protein tagging probes do not need to change their chemical structures during labelling different subcellular structures, which can avoid potential interference caused by chemical targeting ligands. Nowadays, SNAP-tag ${ }^{32}$ (based on benzylguanine-ligated substrates and mutant human $\mathrm{O}^{6}$ alkylguanine-DNA alkyltransferase (AGT) fusion proteins) and HaloTag $^{33}$ (based on alkyl chlorides and dehalogenase enzymes) are the most widely investigated protein labelling technologies (Fig. 1F).

\subsection{Detection mechanisms toward RONSS}

As the most reactive bioactive species in living cells, RONSS are responsible for intracellular redox homeostasis. Any uncontrolled expression of RONSS could cause oxidative, nitrative or reductive stress owing to the capabilities of these molecular species in reacting with diverse biomolecules, such as DNA, lipids and enzymes. ${ }^{3 c}$ Such imbalances can hamper the smooth progress of different biological activities and further result in a series of major diseases, such as cardiovascular disease, neurodegenerative disease, diabetes, inflammation and can-

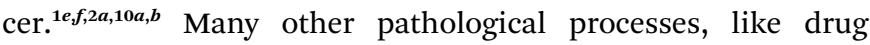
induced liver injury (DILI), ischemia reperfusion injury (IRI) and so on, are also accompanied by the overproduction of RONSS. ${ }^{3 c}$ More importantly, an increasing number of research studies have revealed that RONSS can be potential messengers for cell signalling, which further spurred investigations into the development of efficient tools for tracing subcellular concentrations of diverse RONSS. ${ }^{3 a, d}$

Among the diverse detection strategies, fluorescence imaging represents a simple, sensitive and non-invasive technique, which enables real-time, dynamic and selective imaging of numerous targets in living cells, and has greatly advanced biological analysis, clinical drug discovery and so on..$^{1 b, g, 3 a, 7 a, 10 a, 21 b}$ Intracellular RONSS feature short life span, low concentration, high reactivity and environmental sensitivity, and they are non-emissive. Therefore the specific imaging of RONSS cannot leave out efficient fluorescent probes. ${ }^{\mathbf{1 d , e , 4 b , 1 1}}$ Up to now, a series of probes based on different reaction mechanisms have been developed for selective labelling of RONSS in living cells. A summary of the detection mechanisms of organelle targeted fluorescent probes toward RONSS is shown in Table 1.

\subsection{Response mechanisms of RONSS fluorescent probes}

After reacting with RONSS, the signal outputs of the fluorescent probes can give appropriate changes via specific mechanisms that can subsequently be detected by a detector. In general, fluorescence resonance energy transfer (FRET), photo-induced electron transfer (PET), intramolecular charge transfer (ICT) and excited-state intramolecular proton transfer (ESIPT) are the most popular response mechanisms for organelle-targeted fluorescent probes.

2.3.1. FRET-based probes. FRET is an energy transfer phenomenon between two or more chromophores. The "donor (D)" can transfer excited state energy to the nearby "acceptor (A)" in a non-radiative manner through long-range dipoledipole interactions due to their spectral overlap, which enables the excitation of "A" chromophores with the excitation wavelength of "D". ${ }^{60}$ Strategies for designing FRET-based probes include (1) constructing probes with cleavable linkers, and (2) synthesizing probes from analyte-responsive $\mathrm{D}$ and $\mathrm{A}$ (Fig. 2A) ${ }^{45,61}$ By virtue of the stoichiometric relation between "D" and "A", FRET-based probes are sensitive and can be employed for ratiometric imaging. The energy transfer efficiency of FRET probes is highly dependent on the spectral overlap efficiency between " $D$ " and " $A$ ". However, the available FRET pairs are still rare.

2.3.2. PET-based probes. PET is an intramolecular electron transfer phenomenon. Probes based on PET can be divided into a-PET and d-PET: a-PET represents the electron transfer from "D" to the excited fluorophore and causes fluorophore reduction as well as fluorescence quenching, and is also called 
Table 1 Detection mechanisms of organelle-targeted fluorescent probes toward RONSS

\begin{tabular}{|c|c|c|c|}
\hline \multicolumn{2}{|c|}{ Bioactive species } & \multirow{2}{*}{$\begin{array}{l}\text { Detection mechanisms } \\
\text { Nucleophilic substitution }\end{array}$} & \multirow{2}{*}{$\frac{\text { Ref. }}{24 a \text { and } 34}$} \\
\hline \multirow[t]{8}{*}{ ROS } & $\mathrm{O}_{2}^{\cdot-}$ & & \\
\hline & & Oxidation of benzothiazoline & 35 \\
\hline & & Oxidation of a diketone & $36 c$ \\
\hline & & Oxidation of phenol & 37 \\
\hline & $\mathrm{HOCl}$ & Oxidation of double bonds $(\mathrm{C}=\mathrm{N}, \mathrm{C}=\mathrm{C}$ and $\mathrm{C}=\mathrm{S})$ & 38 \\
\hline & ${ }^{1} \mathrm{O}_{2}$ & Formation of an endoperoxide & 41 \\
\hline & $\cdot \mathrm{OH}$ & Oxidation of phenothiazine & 42 \\
\hline & $\mathrm{HOBr}$ & Catalytic cyclization between the amino and $S$-methyl group & 43 \\
\hline \multirow[t]{3}{*}{ RNS } & $\mathrm{ONOO}^{-}$ & Oxidation of phenylhydrazine & 44 \\
\hline & & Oxidation of an unsaturated bond & 45 \\
\hline & & Deboronation & 46 \\
\hline \multirow[t]{9}{*}{ RSS } & GSH & Michael addition & 51 \\
\hline & & Nucleophilic substitution & 52 \\
\hline & Cys & Michael addition & 53 \\
\hline & $\mathrm{H}_{2} \mathrm{~S}$ & Reduction of an aryl azide/nitro group & $26 b$ and 54 \\
\hline & & Thiolysis & 55 \\
\hline & & $\mathrm{Cu}^{2+}$ precipitation & 56 \\
\hline & & Nucleophilic addition & 57 \\
\hline & $\mathrm{H}_{2} \mathrm{~S}_{n}$ & Nucleophilic aromatic substitution & 58 \\
\hline & $\mathrm{SO}_{2}$ & Nucleophilic addition & 59 \\
\hline
\end{tabular}

reductive-PET; on the other hand, d-PET is the process of electron transfer from an excited fluorophore to the electron deficient "A", and is also called oxidative-PET (Fig. 2B). PET-based probes are usually characterized by "turn-on", which is beneficial for fluorescence imaging with a high signal-to-noise ratio. ${ }^{62}$ Nevertheless, traditional PET-based probes cannot quantify analytes based on the fluorescence intensity change. To solve this problem, an extra inner reference fluorophore can be introduced during designing functional probes.

2.3.3. ICT-based probes. ICT based probes are constructed by directly conjugating " $\mathrm{D}$ " and "A" with fluorophores. This "D$\pi-A$ " structure results in strong "push-pull" effects within fluorophores, which is beneficial for fluorescence intensity enhancement and emission wavelength red shift (Fig. 2C). ${ }^{2 c}$

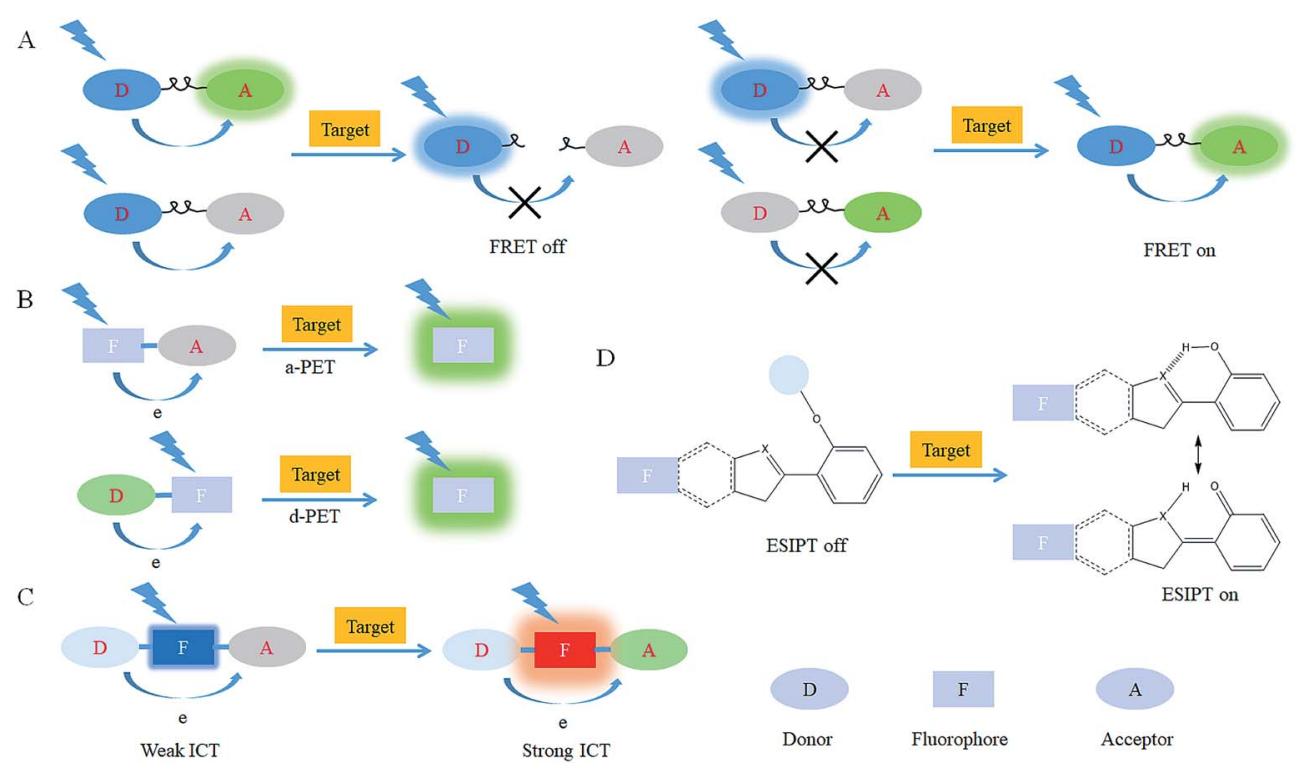

Fig. 2 Response mechanisms for organelle-targeted probe design: (A) FRET, (B) PET, (C) ICT and (D) ESIPT. 
Thus, ICT-based probes can be used to detect analytes with high sensitivity and accuracy. Ideal ICT-based probes should have an apparent fluorescence intensity change and a large emission wavelength shift for effective ratiometric measurement. ${ }^{2 f}$ It should be noted that the lone pair electrons of $\mathrm{N}, \mathrm{O}$, and $\mathrm{S}$ heteroatoms in ICT-based probes may diminish and even quench the initial fluorescence of the fluorophores, being able to further impair the calibration functions of related probes. ${ }^{63}$

2.3.4. ESIPT-based probes. ESIPT refers to the phenomenon of transfer of hydrogen atoms (mainly from hydroxyl or amino groups) in the fluorescent compound to nearby heteroatoms (mainly N, O or S) under laser excitation (Fig. 2D). Fluorophores with ESIPT characteristics can build the fast four-level photocycle between the ketal and enol isomers under excitation. ${ }^{2 e}$ Because of the different energy level structures between the tautomers, ESIPT fluorophores can emit two distinct emission bands with large Stokes shifts, which makes it another attractive strategy for functional fluorescent probe design. ${ }^{2 e}$ However, the ESIPT phenomenon may be restricted in protic environments. ${ }^{64}$ Therefore, in the design of acidic organelle-targeted probes, ESIPTbased probes should be avoided.

\section{Nucleus-targeted probes}

As discussed above, the nucleus is a crucial organelle of eukaryotic cells. An increasing number of reports have proved that RONSS can be important messengers during gene expression. However, only a limited number of nucleus-targeted probes have been devised up to now, though they are favourable for further revealing/verifying the potential biological functions of these molecules (Fig. 3).

\subsection{Imaging of ROS in the nucleus}

ROS can diffuse into the nucleus and regulate numerous critical physiological processes, while excess ROS can cause DNA damage/mutation and disrupt nuclear homeostasis. ${ }^{3 c}$ Thus tracing the nuclear ROS concentration holds promise for revealing the molecular mechanisms of some related biological events. However, there are only limited reports on nucleustargeted ROS imaging up to now, most likely because it is challenging to drive probes into the nucleus effectively and selectively image the bioactive molecules of interest at ultralow concentrations.

By screening an arylboronate based probe, Chang's research group discovered a nucleus-targeted $\mathrm{H}_{2} \mathrm{O}_{2}$ probe, 1 (NucPE1). ${ }^{17}$ Interestingly, although without a specific targeting moiety, this probe can accumulate in the nucleus of several mammalian cell lines and C. elegans. Moreover, once 1 reacted with $\mathrm{H}_{2} \mathrm{O}_{2}$ in the cytoplasm, it lost its targeting capability, which enables the nucleus-selective $\mathrm{H}_{2} \mathrm{O}_{2}$ imaging. Although the targeting mechanism remains to be further clarified, this probe can serve as a novel fluorescent tool for monitoring the nucleus $\mathrm{H}_{2} \mathrm{O}_{2}$ fluxes. Importantly, they revealed the relationships between sir-2.1 overexpression and enhanced regulation of nuclear ROS pools with this probe, which proved that the longevity is closely related to the ROS regulation capacity.
Using nuclear-localization signal peptides (NLS, detailed structure shown in Fig. 1A) as the targeting moiety, Yi et al. successfully prepared a naphthalimide based probe, 2 (pepNP1), for nucleus-targeted $\mathrm{H}_{2} \mathrm{O}_{2}$ imaging. ${ }^{19}$ Confocal laser scanning microscope (CLSM) experiments indicated the successful nuclear localization of 2, while probe NP1 without NLS peptide functionalization was retained in the cytoplasm. 2 was used for ratiometric imaging of the intra-nuclear $\mathrm{H}_{2} \mathrm{O}_{2}$ in HeLa cells (Fig. 4).

Protein tagging is another effective strategy for delivering probes into the nucleus. Chang's group reported SNAP-tag based $\mathrm{H}_{2} \mathrm{O}_{2}$ probes 3 (SPG1, membrane-impermeable, and SPG2, membrane-permeable), which can specifically bind with AGT fused protein. ${ }^{65}$ Upon coming into contact with the localized $\mathrm{H}_{2} \mathrm{O}_{2}$, 32-fold turn-on fluorescence can be detected due to the deprotection of boronate groups. By transfecting different plasmids, $\mathrm{H}_{2} \mathrm{O}_{2}$ in the nucleus, mitochondria, plasma membrane and ER of living cells was detected with the probes, which offered a versatile fluorescent platform for tracing the subcellular $\mathrm{H}_{2} \mathrm{O}_{2}$ level.

\subsection{Imaging of RNS in the nucleus}

In addition to ROS, RNS are also critical bioactive species for living organisms. Due to the ultrashort lifespan and diffuse distance of RNS, the action sites of these molecules are usually determined by their locations. Therefore, precise imaging of the subcellular RNS distribution will accelerate our understanding of the biological functions of these molecules.

NO is an important signalling molecule that can exert multiple biological effects on living cells, and it has also been widely investigated for cancer treatment. Clarifying the function and identifying the target sites of NO can facilitate related research studies. By taking advantage of the HaloTag technology, Yang and $\mathrm{Hu}$ et al. developed an organelle-targetable probe, 4 (HTDAF-2DA). ${ }^{66}$ The probe has excellent cell permeability due to the introduction of diacetate groups. After entering living cells, the ester groups were hydrolyzed and the probe was subsequently immobilized by HaloTag protein. Upon the addition of exogenous NO, the fluorescence of probes can be turned on due to the inhibition of the PET process after formation of a triazole product. Based on different plasmids, the probe was specifically delivered into the nucleus, cytosol, mitochondria and plasma membrane. The target site of exogenous NO was successfully revealed to be the plasma membrane with 4.

By connecting the rhodamine derivative with the SNAP-tag substrate, Xiao's group reported another organelle-targetable genetically encoded NO probe, 5 (TMR-NO-SNAP), which has been used for imaging the endogenous subcellular NO generation in LPS/IFN- $\gamma$ stimulated RAW264.7 cells and etoposide treated COS-7 cells. $^{67}$

\subsection{Imaging of RSS in the nucleus}

RSS are another group of bioactive species closely related to cellular redox homeostasis. Benefiting from the reductive activity of these species, RSS can interact with diverse proteins 

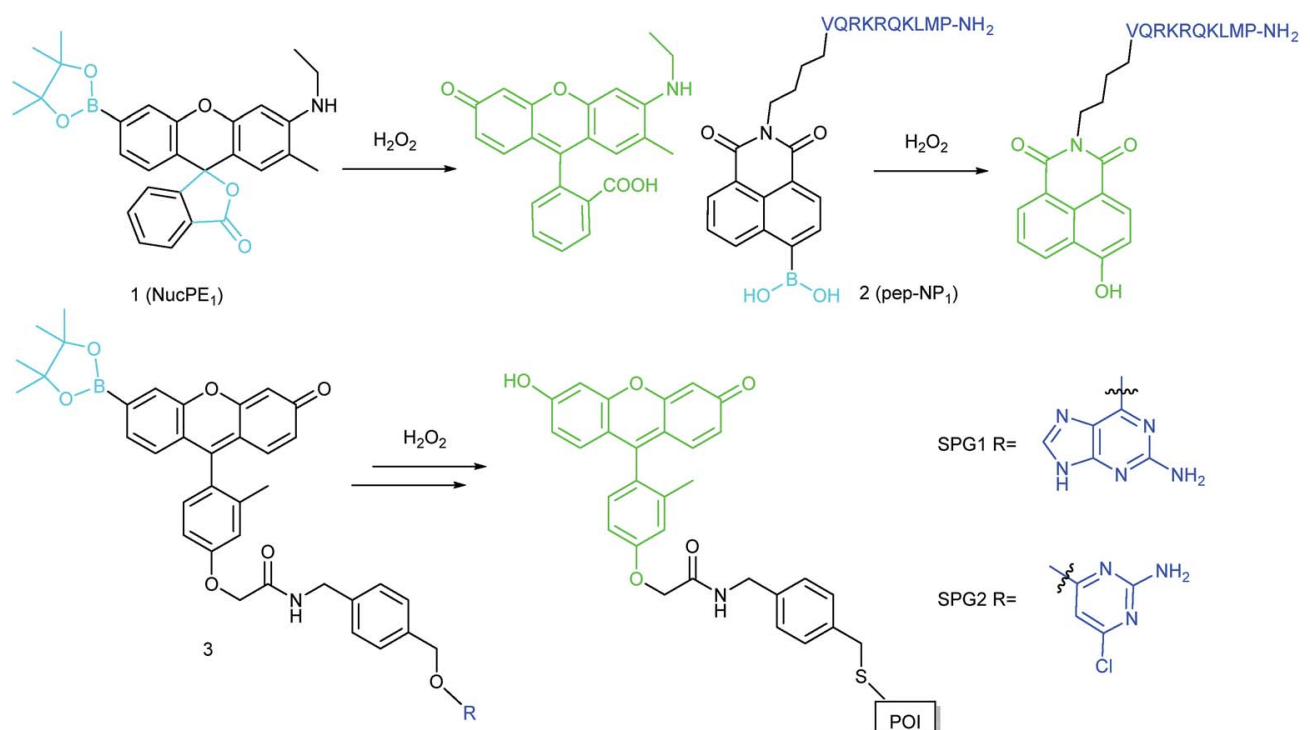<smiles></smiles><smiles>[R]C(=O)CNc1cc2c(cc1N)C1(OC2=O)c2ccc(OC(C)=O)cc2Oc2cc(OC(C)=O)ccc21</smiles><smiles>Nc1cc(-c2c3ccc(=O)cc-3oc3cc(O)ccc23)c(C(=O)O)cc1NCC(=O)O</smiles><smiles>[R]C(=O)Cn1nnc2cc(-c3c4ccc(=O)cc-4oc4cc(O)ccc34)c(C(=O)O)cc21</smiles>

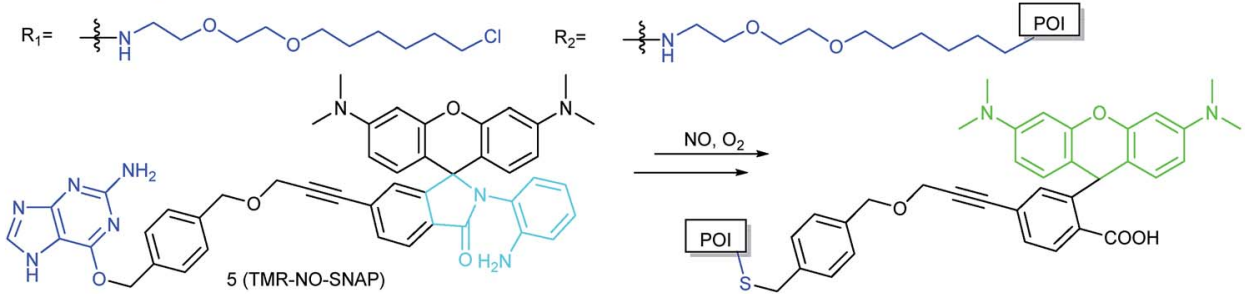<smiles>CC(C)(C)OCc1ccc(CNC(=O)c2cc3ccc(N)cc3oc2=O)cc1</smiles><smiles></smiles>

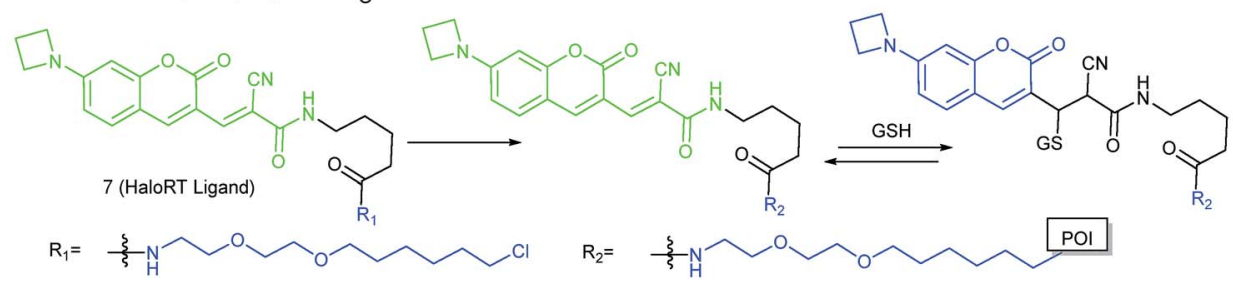

Fig. 3 Fluorescent probes for imaging RONSS in the nucleus.

within the nucleus, which can further influence the gene expression. Therefore, mapping the intra-nuclear concentration and distribution of RSS is another significant task for investigating the redox biology in the nucleus.

In 2016, Wang's group reported a genetically encoded coumarin based probe, 6 ( $\left.\mathrm{CouN}_{3}-\mathrm{BC}\right)$, for nucleus- and mitochondrion-targeted $\mathrm{H}_{2} \mathrm{~S}$ imaging. ${ }^{68}$ After transfecting pCLIP-H2B and pCLIP-COX8A plasmids, ${ }^{69}$ the tag protein was successfully fused with $\mathrm{H} 2 \mathrm{~B}$ protein (in the nucleus) and a cytochrome $\mathrm{c}$ oxidase subunit (in the mitochondrial inner membrane and matrix), respectively. Subsequently, probe $\mathrm{CouN}_{3}$-BC was selectively anchored to the nucleus or mitochondria and further lit up localized $\mathrm{H}_{2} \mathrm{~S} . \mathrm{H}_{2} \mathrm{~S}$ accumulation in the nucleus of HeLa cells under NO donor sodium nitroprusside stimulation was successfully tracked with this probe. In 2018, they proposed another genetically encoded probe, 7 (HaloRT Ligand), for reversible GSH imaging. ${ }^{70}$ Based on the HaloTag technique, 7 was successfully labelled in the nucleus, mitochondria, ER, and cytosol. And they revealed for the first time that under various stimulations, the GSH concentrations 


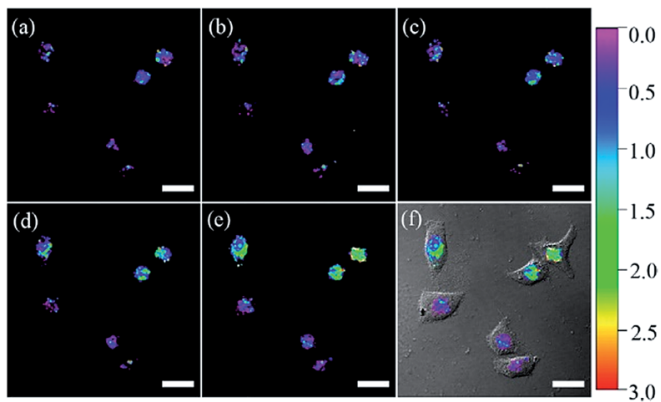

Fig. 4 CLSM ratio images of HeLa cells loaded with $50 \mu \mathrm{M} 2$ and stimulated with $200 \mu \mathrm{M} \mathrm{H}_{2} \mathrm{O}_{2}$ for (a) 0, (b) 15, (c) 30, (d) 60 , and (e) $75 \mathrm{~min}$ at $37^{\circ} \mathrm{C}$. (f) The overlay image of (e) and the brightfield image. Adapted with permission from ref. 19. Copyright 2014 American Chemical Society.

in the nucleus and cytosol remained nearly the same, which suggested that there is no GSH pool in the nucleus.

\subsection{Challenges for nucleus-targeted fluorescent probes}

Several reports have suggested that the conjugation of molecular or nanomaterials with NLS peptides can offer them the capability for nucleus specific accumulation, and nucleustargeted cancer therapy has been widely investigated. ${ }^{16 a, 18,71}$ However, novel nucleus-targeted fluorescent probes are still rare, and due to the fact that the targeting efficiency of those materials is unsatisfactory, many probes require long incubation time for nuclear localization. Moreover, direct conjugation of probes with nucleus-targeted molecular scaffolds or NLS peptides is usually unsuccessful, most likely because minor structural alterations can reduce the affinity of the targeting ligands. ${ }^{3 a}$ Protein tagging seems to be an ideal strategy for anchoring probes into almost any organelles including the nucleus. But the synthetic difficulty and complexity, and loss of solubility of probes after binding with the substrates can hinder the application of genetically encoded probes. Excess un-tagged probes during imaging must be washed out several times to reduce the background fluorescence, which can hamper the activity and change the subcellular microenvironments of cells. Moreover, only the RONSS near the fusion protein can be detected by the probes, which further diminished the applications of genetically encoded probes. Thus, more attention should be paid to establish novel methodologies for nucleustargeted ultrasensitive probe design.

\section{Mitochondrion-targeted probes}

Mitochondria are the energy center of living cells, and they produce the majority of ATP for numerous biological events, including cell proliferation, division, biomolecule fabrication and so on. ${ }^{24 d}$ Therefore, the mitochondrial function and homeostasis determine the physiological and pathological processes of living cells. RONSS are closely related to the signal conducting and mitochondrial microenvironments. ${ }^{72}$ The past decade has witnessed significant progress of mitochondrion targeted fluorescent probes for localized RONSS imaging, owing

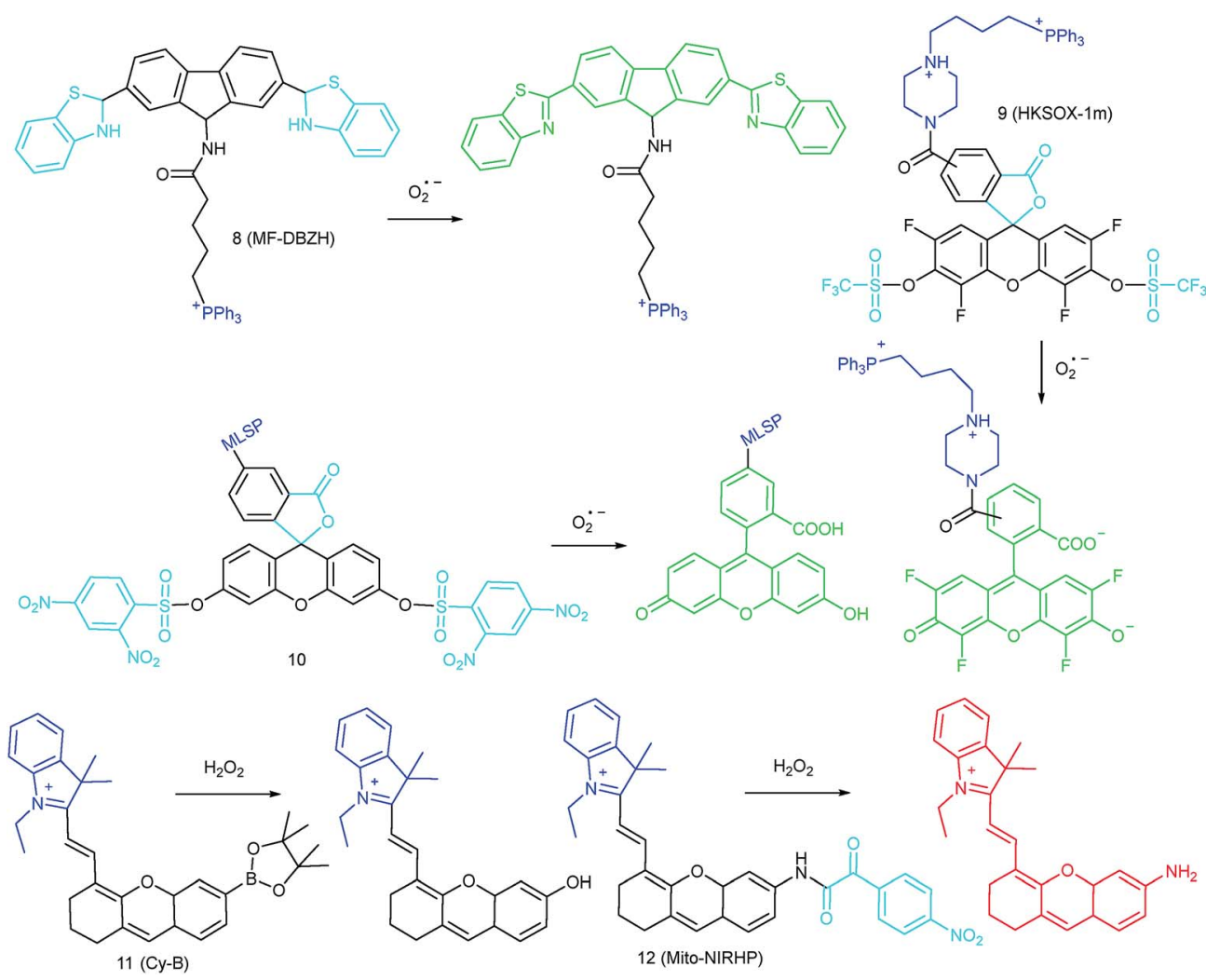

Fig. 5 Mitochondrion-targeted probes for $\mathrm{O}_{2}{ }^{\cdot-}$ and $\mathrm{H}_{2} \mathrm{O}_{2}$ imaging. 
to its potential in offering theoretical bases for disease diagnosis and therapy (Fig. 5).

\subsection{Imaging of ROS in mitochondria}

To ensure intracellular energy supply, mitochondria perform diverse electron-transport chain (ETC) reactions for ATP synthesis. Once the electrons leak from the ETC, they can react with oxygen molecules to generate $\mathrm{O}_{2}{ }^{\cdot-}$. Under the catalysis of diverse enzymes, $\mathrm{O}_{2}{ }^{-}$can be further transformed into $\mathrm{H}_{2} \mathrm{O}_{2}$, $\mathrm{ONOO}^{-},{ }^{\circ} \mathrm{OH},{ }^{1} \mathrm{O}_{2}$ and so on. ${ }^{23 a, b, 35,43 a, 44}$ These bioactive species can serve not only as oxides to maintain the mitochondrial redox homeostasis, but also as signal molecules to regulate the cellular function. However, once overexpressed, they can cause serious oxidative stress and further hamper the mitochondrial function, resulting in diverse disorders as well as diseases. ${ }^{2 g, 3 d, 7 a, 38 b, 39 a, 43 b}$ In the past decade, a variety of mitochondrion specific fluorescent probes have been developed for tracing ROS fluctuations during diverse physiological and pathological processes.

4.1.1. Imaging of $\mathrm{O}_{2}{ }^{-{ }^{-}}$in mitochondria. $\mathrm{O}_{2}{ }^{-{ }^{-}}$is the reaction product of oxygen and the electrons leaked from the mitochondrial ETC; it can also be generated during monoamine oxidase directed oxidative deamination. The intracellular concentration of $\mathrm{O}_{2}{ }^{--}$is about $10^{-10} \mathrm{M}$, and the half-life is $10^{-6} \mathrm{s.}^{3 c}$ Because $\mathrm{O}_{2}{ }^{-}$is the precursor of a series of bioactive species, its concentration determines the levels of the downstream species to some extent. Precise imaging of the mitochondrial $\mathrm{O}_{2}{ }^{--}$concentration plays a critical role in the understanding of its physiological and pathological functions.

Inspired by the mitochondrion targeting capability of the cationic lipophilic group TPP, our group developed a twophoton (TP) fluorescent probe, 8 (MF-DBZH), for mitochondrial endogenous $\mathrm{O}_{2}{ }^{--}$detection. ${ }^{35}$ The probe is composed of TPP, $\mathrm{O}_{2}{ }^{-}$sensitive benzothiazoline and TP fluorophore fluorene. Upon the addition of $\mathrm{O}_{2}{ }^{--}$to a solution of $\mathbf{8}$, the specific benzothiazoline dehydrogenation reaction results in an extended conjugate plane, leading to TP fluorescence enhancement due to the disruption of the PET process. The limit of detection (LOD) of this probe was calculated to be $9.5 \mathrm{nM}$. Living cell co-staining revealed that 8 effectively localized into the mitochondria under TPP guidance. This $\mathrm{pH}^{-}$ insensitive and photostable TP probe has been used to selectively image the mitochondrial endogenous $\mathrm{O}_{2}{ }^{\cdot-}$ fluctuation during the inflammation process at cellular and living body levels with the assistance of TP microscopy (Fig. 6).

Another TPP functionalized mitochondrion-targeted $\mathrm{O}_{2}{ }^{\cdot-}$ probe, 9 (HKSOX-1m), was reported by Yang's group. ${ }^{34} 9$ employs fluorescein as the fluorophore and aryl trifluoromethanesulfonate as the recognition group. The cleavage of the aryl trifluoromethanesulfonate group and rearrangement reaction during the nucleophilic reaction between 9 and $\mathrm{O}_{2}{ }^{--}$ resulted in dramatic fluorescence intensity enhancement. The emission intensity of $\mathbf{9}$ increased linearly upon the addition of $\mathrm{O}_{2}{ }^{\cdot-}$ into the probe solution. The mitochondrial $\mathrm{O}_{2}{ }^{\cdot-}$ concentration fluctuation in human THP-1 cells in the resting state or under antimycin A stimulation was monitored with this probe.

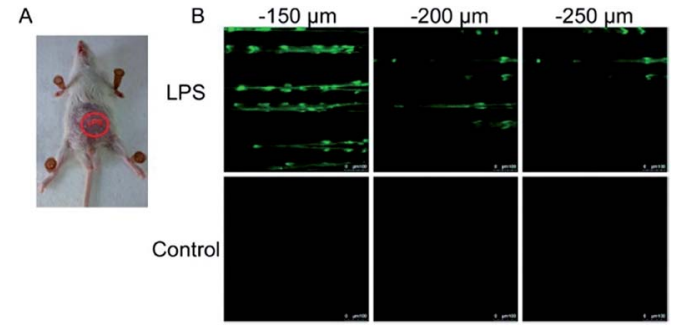

Fig. 6 TP microscopy images of mice with LPS-mediated abdomen injury using probe 8 . (A) $\mathrm{O}_{2}{ }^{--}$was produced inside the peritoneal cavity of the mice during the LPS-mediated inflammatory response. (B) TP fluorescence in situ images of mice abdomen incubated with 8 (100 $\mu \mathrm{M})$. Adapted with permission from ref. 35. Copyright 2013 American Chemical Society.

9 also showed promise for revealing other biological and pathological functions of $\mathrm{O}_{2}{ }^{\cdot-}$.

Apart from using TPP as the localization group, peptides have also been employed for mitochondrion-specific probe design. ${ }^{24 a}$ Zhong's group reported a mitochondrial localization signal peptide (MLSP) functionalized $\mathrm{O}_{2}{ }^{--}$probe, 10, which has been successfully used for monitoring mitochondrial $\mathrm{O}_{2}{ }^{-}$ generation in RAW264.7 macrophages under the stimulation of chemical molecules or single-walled carbon nanotubes. ${ }^{24 a}$

4.1.2. Imaging of $\mathrm{H}_{2} \mathrm{O}_{2}$ in mitochondria. $\mathrm{O}_{2}{ }^{-{ }^{-}}$can convert into $\mathrm{H}_{2} \mathrm{O}_{2}$ under the catalysis of superoxide dismutase (SOD), which has lower activity than other ROS, but serves as an important messenger for signal conduction. ${ }^{23 b, 73}$ The half-life of $\mathrm{H}_{2} \mathrm{O}_{2}$ is $10^{-5} \mathrm{~s}$ and its intracellular concentration is about $10^{-7} \mathrm{M}$. Investigations revealed that the intracellular concentration of $\mathrm{H}_{2} \mathrm{O}_{2}$ is much higher in diseased cells, such as diverse cancer cells. Therefore, $\mathrm{H}_{2} \mathrm{O}_{2}$ is another important biomarker to be monitored. Tracing the spatial distribution and concentration fluctuation of $\mathrm{H}_{2} \mathrm{O}_{2}$ in the mitochondria is beneficial for understanding the pathogenesis of $\mathrm{H}_{2} \mathrm{O}_{2}$-associated diseases and further exploring novel drugs for clinical therapy.

Generally speaking, aryl boronates and phenylboronic acid are the most widely employed groups for $\mathrm{H}_{2} \mathrm{O}_{2}$ specific probes. Peng's group reported a hemicyanine based $\mathrm{H}_{2} \mathrm{O}_{2}$ probe, $\mathbf{1 1}$ (CyB), which was prepared by direct modification of hemicyanine dye with a borate ester moiety. ${ }^{36 a}$ The cationic feature of $\mathbf{1 1}$ makes it specifically accumulate in the mitochondria (overlapped with rhodamine 123, Pearson's coefficient $=0.98$ ), while the mitochondrial $\mathrm{H}_{2} \mathrm{O}_{2}$ can specifically oxidize the boronate moiety and cause the "turn-on" of fluorescence. 11 was successfully employed for imaging mitochondrial $\mathrm{H}_{2} \mathrm{O}_{2}$ during autophagy induced by rapamycin. Benefiting from its NIR emissive behavior, in vivo $\mathrm{H}_{2} \mathrm{O}_{2}$ imaging was also demonstrated.

IRI occurs in many cases, including traumatic shock, surgery, organ transplantation, frostbite, thrombosis and so on, and can cause deadly damage to organs. Recent research studies have revealed that ROS are associated with IRI. Fluorescent probes capable of monitoring the subcellular ROS level during IRI are critical for understanding the pathogenesis and may further accelerate the related drug discovery. By using $\alpha$ ketoamide as the responsive moiety, Our group developed 


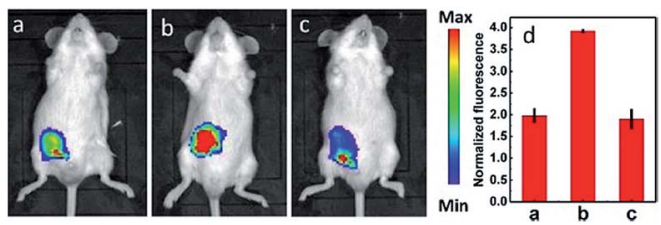

Fig. 7 Fluorescence imaging (pseudocolor) of $\mathrm{H}_{2} \mathrm{O}_{2}$ in Kunming mice. (a) Only $12(20 \mu \mathrm{M}, 100 \mu \mathrm{L})$ was injected as a control. (b) Mouse pretreated with rotenone for $1 \mathrm{~h}$ and then injected with 12. (c) Mouse successively injected with rotenone, NAC, and 12. (d) Relative fluorescence intensities of $(a-c)$. Adapted with permission from ref. $36 c$. Copyright 2013 American Chemical Society.

another hemicyanine-based mitochondrion-targeted $\mathrm{H}_{2} \mathrm{O}_{2}$ probe, 12 (Mito-NIRHP). ${ }^{36 c}$ Benefiting from the ICT mechanism as well as the specific response capability of $\alpha$-ketoamide, $\mathbf{1 2}$ was used for $\mathrm{H}_{2} \mathrm{O}_{2}$ imaging under both in vitro and in vivo conditions (Fig. 7). Importantly, with this probe, the relationship between $\mathrm{H}_{2} \mathrm{O}_{2}$ and kidney IRI was elucidated in subcellular, organ, and living body levels.

4.1.3. Imaging of $\mathbf{H O C l}$ in mitochondria. Hypochlorous acid ( $\mathrm{HOCl}) / \mathrm{OCl}^{-}$can be produced from $\mathrm{H}_{2} \mathrm{O}_{2}$ and $\mathrm{Cl}^{-}$via catalysis by heme enzyme myeloperoxidase (MPO). ${ }^{38 a}$ They are closely related to cellular immunity because of their high reactivity. An appropriate level of $\mathrm{HOCl} / \mathrm{OCl}^{-}$in the mitochondria can defend the host cells from infection caused by a variety of pathogens. Overproduction of $\mathrm{HOCl} / \mathrm{OCl}^{-}$may cause a series of problems due to their reactivity, which can break a broad spectrum of biomolecules, including DNA, lipids, proteins, iron-sulfur clusters, and thiols, among others. ${ }^{40 b}$ Numerous diseases, like cancer, inflammation and atherosclerosis, are accompanied by $\mathrm{HOCl}$ overexpression. Mitochondrial HOCl/

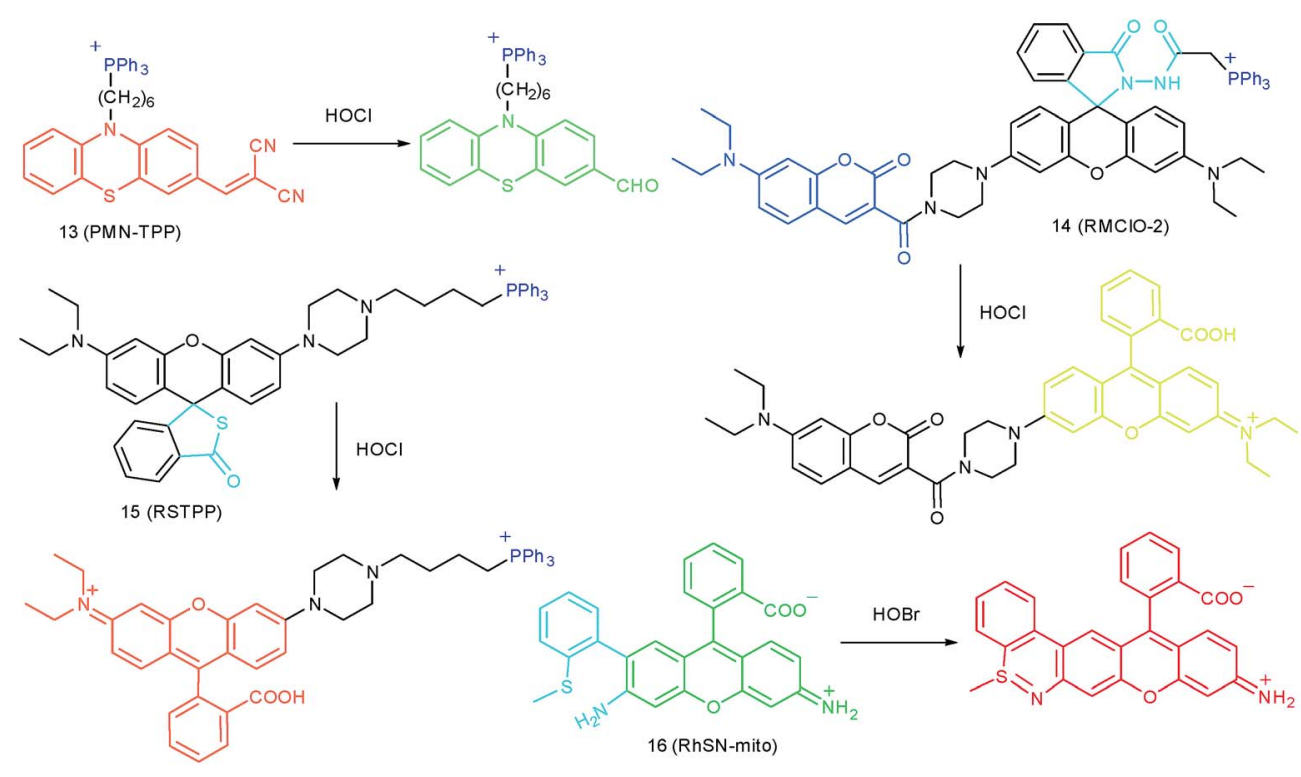
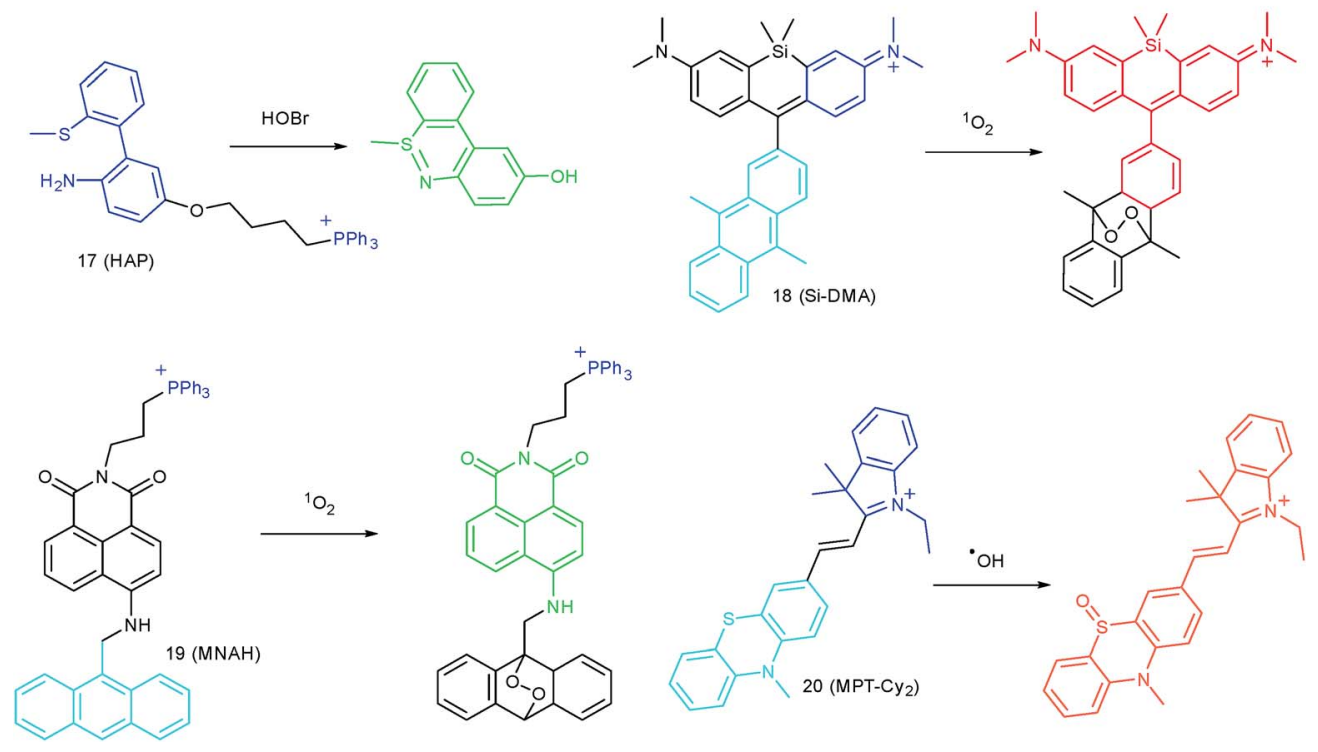

Fig. 8 Mitochondrion-targeted probes for $\mathrm{HOCl}, \mathrm{HOBr},{ }^{1} \mathrm{O}_{2}$ and $\cdot \mathrm{OH}$ imaging. 
$\mathrm{OCl}^{-}$imaging is therefore of great significance. In the past, many reaction mechanisms have been proposed for detecting $\mathrm{HOCl}$ in living cells, typically including oxidative cleavage of double bonds $(\mathrm{C}=\mathrm{N}, \mathrm{C}=\mathrm{C}, \mathrm{C}=\mathrm{S}$ and $\mathrm{N}=\mathrm{N})$, lactams, and thiolactones, and oxidation of thioethers and selenides (Fig. 8). ${ }^{38 a, b, 39,40 c, 74}$

Wang's group reported 13 (PMN-TPP) as a colorimetric and ratiometric probe for $\mathrm{HOCl}$ imaging. ${ }^{38 c}$ In the presence of $\mathrm{HOCl}$, the specific oxidative cleavage of $\mathrm{C}=\mathrm{C}$ disrupts the conjugated structure and disturbs the ICT process, which results in a decrease in the fluorescence at $640 \mathrm{~nm}$ as well as the appearance of a new emission peak at $522 \mathrm{~nm}$. Confocal imaging experiments revealed that $\mathbf{1 3}$ localized well in the mitochondria of macrophages due to the TPP functionalization. 13 has been successfully used for ratiometric imaging of endogenous $\mathrm{HOCl}$ in nude mice with acute inflammation (Fig. 9).

Apart from double bonds, many other groups have also been introduced for mitochondrion-targeted HOCl probe design. By using rhodamine as the fluorophore and benzoyl acetohydrazide as the responsive group, Yu's group developed several mitochondrion specific $\mathrm{HOCl}$ probes. ${ }^{39 a, 75}$ Based on the FRET pair of coumarin and rhodamine, TPP modified probe 14 (RMClO-2) was facilely prepared. ${ }^{39 a} 14$ has excellent membrane permeability, mitochondrion localization capacity, and $\mathrm{HOCl}$ selectivity, with an ultralow LOD of $0.84 \mathrm{nM}$. Benefiting from these features, $\mathbf{1 4}$ was used to quantify the basal mitochondrial $\mathrm{HOCl}$ of cancer cells for the first time.

As important immune cells, macrophages play a critical role in the innate immune system. Though it has been reported that $\mathrm{HOCl}$ could be detected in the cytoplasm, it was unclear whether $\mathrm{HOCl}$ can appear in the mitochondria during infection before $\mathrm{Ma}$ and co-workers developed the rhodamine based probe 15 (RSTPP). ${ }^{39 b}$ The fluorescence quantum yield of $\mathbf{1 5}$ remained low $(<0.01)$, but was elevated to 0.34 in the presence of $\mathrm{HOCl}$, with a $>200$-fold fluorescence intensity enhancement due to the oxidative cleavage of the thiolactone ring and formation of a large conjugated plane. 15 exhibits high specificity toward HOCl over other ROS with a LOD of $9 \mathrm{nM}$. Confocal laser fluorescence experiments revealed that this TPP decorated rhodamine thiolactone probe localizes at the mitochondria of RAW264.7 cells specifically. The HOCl fluctuation in

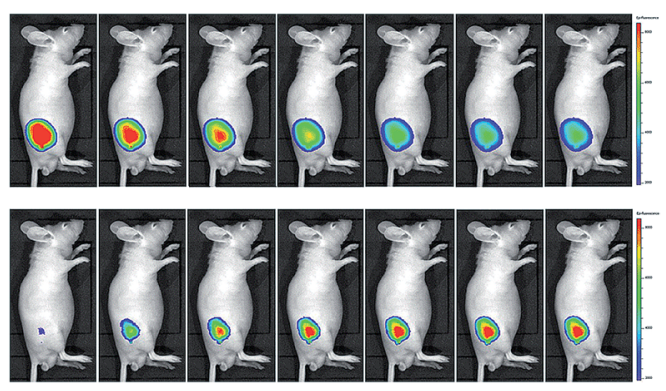

Fig. 9 In vivo dual channel imaging of LPS and PMA treated nude mice with probe 13. Images were taken after stimulation for $0,5,10,15,20$, 30, 45 and $60 \mathrm{~min}$, respectively. Adapted with permission from ref. 38c. Copyright 2015 Royal Society of Chemistry. macrophages during microbial infection was visualized for the first time, which further proved the great potential of subcellular localized probes for bioanalysis.

4.1.4. Imaging of $\mathrm{HOBr}$ in mitochondria. $\mathrm{HOBr}$ is another important ROS that is responsible for numerous physiological and pathological processes. But the ultralow concentration, high reactivity and short life span of $\mathrm{HOBr}$ make it hard for it to be visualized in living cells. Based on the catalytic cyclization reaction between the amino group and $S$-methyl group, our group reported the first $\mathrm{HOBr}$ probe BPP, with high selectivity and ultralow LOD (17 pM), for imaging endogenous $\mathrm{HOBr}$ under both in vitro and in vivo conditions. ${ }^{76}$

Later, a mitochondrion-targeted $\mathrm{HOBr}$ probe, 16 (RhSNmito), was developed. Rhodamine 110 was employed as the fluorophore owing to its high quantum yield, excellent solubility and mitochondrion-targeting capability. ${ }^{43 a} 16$ exhibits a large Stokes shift $(\sim 100 \mathrm{~nm})$ after reacting with $\mathrm{HOBr}$. The quantum yield of the final product was determined to be 0.68 , and the LOD of 16 was 20 pM. Endogenous HOBr in HepG2 cells and zebrafish were tracked with this novel probe (Fig. 10). Very recently, TPP decorated probe 17 (HAP) ${ }^{\mathbf{4 3 b}}$ was devised by Tian's group for tracing mitochondrial HOBr burst in RAW264.7 cells during $\mathrm{O}_{2}{ }^{\cdot-}$ triggered oxidative stress (Fig. 11).

4.1.5. Imaging of ${ }^{1} \mathrm{O}_{2}$ in mitochondria. ${ }^{1} \mathrm{O}_{2}$ can be generated when molecular oxygen is excited to its lowest excited state. ${ }^{1} \mathrm{O}_{2}$ may oxidize numerous biomolecules and cause cell apoptosis, which has been employed in cancer treatment for a long time. ${ }^{77}$ During photodynamic therapy (PDT), ${ }^{1} \mathrm{O}_{2}$ is the main reactive species generated from excited photosensitizers that induces cancer cell death. Evidence also suggested that ${ }^{1} \mathrm{O}_{2}$ participates in signal conduction and gene expression. The fluorescent imaging of ${ }^{1} \mathrm{O}_{2}$ in subcellular structures is quite essential for assessing the therapeutic efficiency and revealing the physiological functions of ${ }^{1} \mathrm{O}_{2}$.

In 2014, Majima and co-workers reported a silicon-containing rhodamine-based probe, 18 (Si-DMA), for mitochondrial ${ }^{1} \mathrm{O}_{2}$
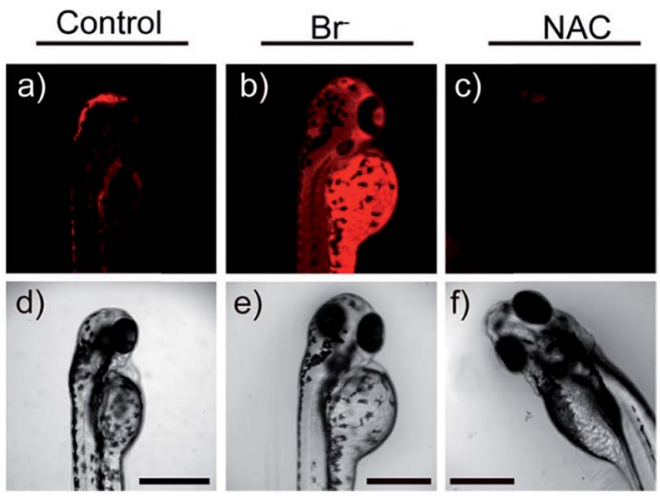

Fig. 10 In vivo fluorescence imaging of $\mathrm{HOBr}$ in zebrafish embryos. (a) Zebrafish fed with 16 for 30 min $(50.0 \mu \mathrm{M})$, (b) zebrafish fed with $\mathrm{Br}^{-}$ $(100 \mu \mathrm{M})$ for $30 \mathrm{~min}$ and then fed with $16(50.0 \mu \mathrm{M})$ for $30 \mathrm{~min}$, and (c) zebrafish incubated with NAC $(20.0 \mu \mathrm{M})$ for $30 \mathrm{~min}$ and then with 16 $(50.0 \mu \mathrm{M})$ for $30 \mathrm{~min}$. (d) - (f) are the corresponding bright-field images of (a)-(c). Adapted with permission from ref. 43a. Copyright 2017 American Chemical Society. 


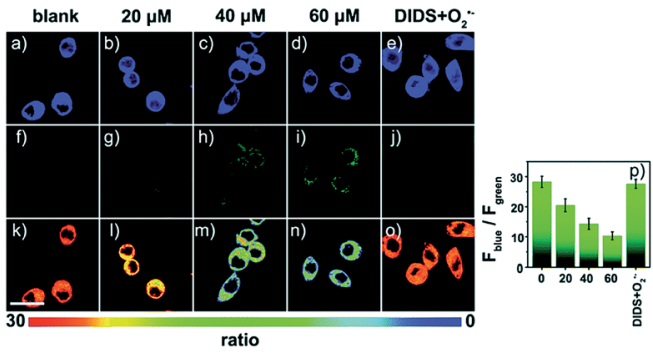

Fig. 11 Confocal fluorescence images of RAW264.7 cells stained with $5 \mu \mathrm{M} 17$ under different treatments: ( $\mathrm{a}, \mathrm{f}$ and $\mathrm{k}$ ) control; ( $\mathrm{b}, \mathrm{g}$ and $\mathrm{l}) 20$ $\mu \mathrm{M} \mathrm{O}_{2}{ }^{\cdot-}$; (c, h and m) $40 \mu \mathrm{M} \mathrm{O}_{2}{ }^{\cdot-}$; (d, i and n) $60 \mu \mathrm{M} \mathrm{O}_{2}{ }^{-{ }^{-}}$; (e, j and o) $100 \mu \mathrm{M}$ DIDS + $60 \mu \mathrm{M} \mathrm{O}_{2}^{\cdot-}$. (p) The corresponding $F_{\text {blue }} / F_{\text {green }}$. Adapted with permission from ref. 43b. Copyright 2018 Royal Society of Chemistry.

imaging. ${ }^{41 b}$ In this probe, 9,10-dimethylanthracene (DMA) is employed as the ${ }^{1} \mathrm{O}_{2}$ recognition moiety, and mitochondrionspecific cationic lipophilic silicon-containing rhodamine (Sirhodamine) serves as the fluorophore. Initially, the fluorescence of rhodamine is quenched due to the PET effect between DMA and Si-rhodamine. During the reaction between 18 and ${ }^{1} \mathrm{O}_{2}$, an endoperoxide is formed between anthracene and ${ }^{1} \mathrm{O}_{2}$, resulting in the disruption of the PET process in the probe, accompanied by fluorescence intensity enhancement. Using this far-red fluorescent probe, the ${ }^{1} \mathrm{O}_{2}$ generation in the mitochondria near the perinuclear region during PDT has been successfully recorded (Fig. 12). Using TPP as the targeting group and naphthalimide as the TP fluorophore, Zhang et al. reported ${ }^{1} \mathrm{O}_{2}$ probe $19(\mathrm{MNAH})^{41 a}$ and successfully visualized the mitochondrial ${ }^{1} \mathrm{O}_{2}$ accumulation during PDT, which offered an alternative tool for tracing PDT in deeper tissues.

4.1.6. Imaging of ${ }^{\circ} \mathrm{OH}$ in mitochondria. $\mathrm{Xu}$ et al. reported a fluorescent probe, 20 (MPT-Cy2), for mitochondrion-targeted 'OH imaging. ${ }^{42}$ Cyanine dye was employed as the mitochondrion-targeted group as well as the fluorophore, while phenothiazine was used as the responsive group owing to its high electron cloud density. During the reaction of 20 and ${ }^{\circ} \mathrm{OH}$, the sulfur atom of phenothiazine was oxidized to sulfoxide, inducing the turn-on of fluorescence with a maximum wavelength of $590 \mathrm{~nm}$. The mitochondrial endogenous ${ }^{\circ} \mathrm{OH}$ in HeLa cells and bacteria was successfully visualized, and the sizedependent ${ }^{\circ} \mathrm{OH}$ generation performance of $\mathrm{TiO}_{2}$ nanoparticles in zebrafish was also revealed with this probe.

\subsection{Imaging of RNS in mitochondria}

As another group of bioactive species for living cells, RNS also has critical impacts on mitochondrial functions. ${ }^{44,45,47,78}$ Tracing the real concentrations of mitochondrial RNS remains challenging, but it is vitally significant for investigating related physiological and pathological processes, which may offer new insights for chemical-biological bioanalysis and clinical disease theranostics.

4.2.1. Imaging of NO in mitochondria. NO is an important cellular messenger molecule that plays critical roles in the nervous, immune, and cardiovascular systems among others.

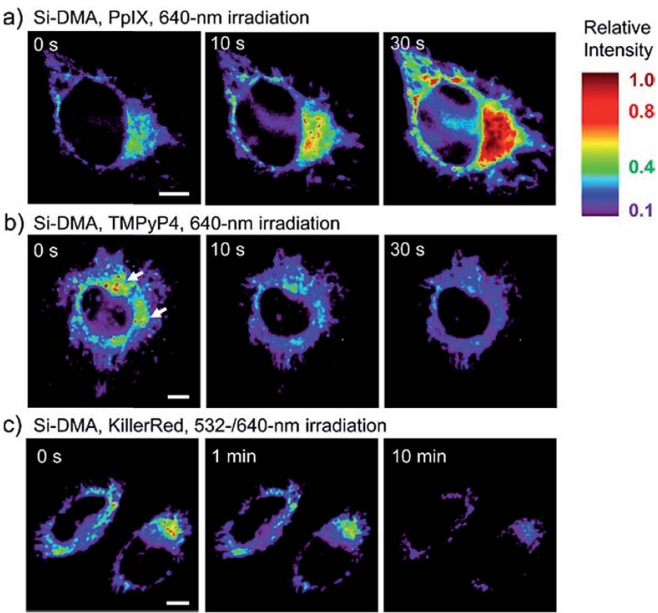

Fig. 12 Fluorescence microscopy images of HeLa cells treated with 18 and (a) 5-ALA induced protoporphyrin IX (PpIX), (b) tetrakis( $N$-methyl4-pyridyl)porphyrin (TMPyP4) and a lysosome marker (A647-dextran), and (c) mitochondrion-targeted KillerRed. ( $a$ and b) Cells were irradiated with a $640 \mathrm{~nm}$ laser to generate ${ }^{1} \mathrm{O}_{2}$ and monitor the fluorescence induced by 18 simultaneously. (c) Two-color irradiation at 532 and $640 \mathrm{~nm}$ was used to excite 18 and KillerRed. Adapted with permission from ref. 41b. Copyright 2014 American Chemical Society.

NO can be synthesized via catalysis by mitochondrial nitric oxide synthases. ${ }^{\mathbf{4} a, \boldsymbol{c}, \mathbf{4 9}}$ The disruption of NO homeostasis results in a series of diseases, including cancer, endothelial dysfunction and neurodegenerative diseases. Monitoring mitochondrial NO is therefore of great significance, and requires efforts in functional probe design. Currently, $o$-phenylenediamine and aromatic secondary amines are the main recognition groups for mitochondrion-targeted NO fluorescent probes.

Jin et al. reported the first mitochondrion-targeted NO probe 21 (Mito-RhNO). ${ }^{48 c}$ This probe employed TPP as the targeting group, rhodamine as the fluorophore and $o$-phenylenediamine as the recognition moiety. The ring-opening of rhodamine spirolactam during the specific reaction between $o$-phenylenediamine and NO caused significant fluorescence enhancement at $585 \mathrm{~nm}$. The LOD of 21 was determined to be $4.0 \mathrm{nM}$. Endogenous mitochondrial NO in the RAW264.7 macrophages under different stimulations was visualized with this biocompatible probe.

Guo et al. demonstrated another fluorescent probe (22) for mitochondrion-targeted NO imaging. ${ }^{48 a} 22$ was prepared by connecting $o$-phenylenediamine with cationic pyronin dye, which could effectively accumulate in the mitochondria. The fluorescence of $\mathbf{2 2}$ was dark due to the PET process. Addition of NO to the solution of 22 induced a 2743-fold fluorescence enhancement at $616 \mathrm{~nm}$, attributed to the triazole generating reaction that lead to the disruption of the PET process. The LOD of 22 was calculated to be $12 \mathrm{nM}$. Interestingly, in the presence of Cys or GSH, a substitution-rearrangement reaction would occur and a bright green or red fluorescence could be generated, ensuring that the mitochondrial NO could be visualized in two distinct emission channels (Fig. 13).

Based on the N-nitrosation of aromatic secondary amines, Miao and coworkers developed mitochondrion-targeted NO 

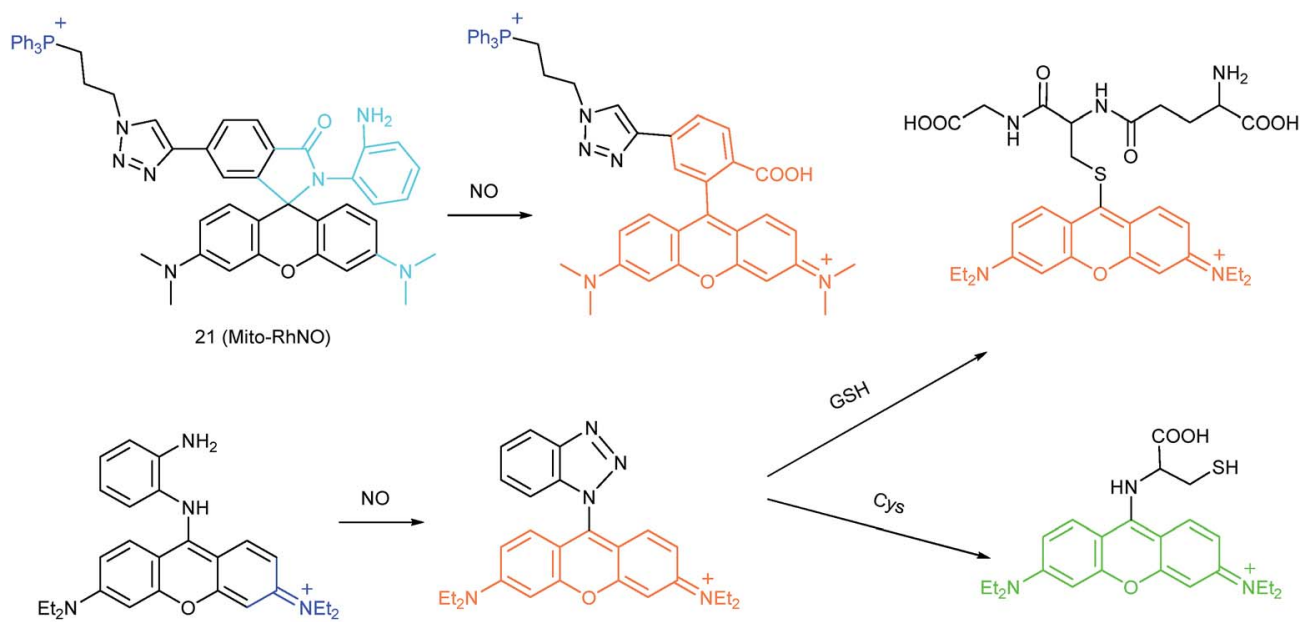

22
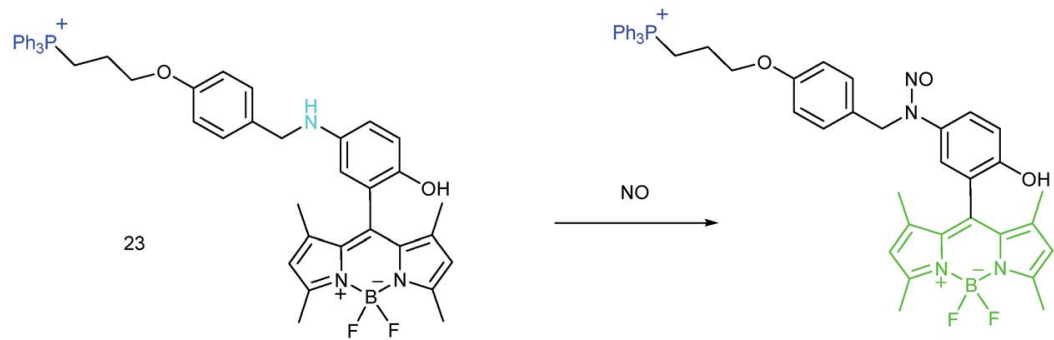

Fig. 13 Fluorescent probes for imaging $\mathrm{NO}$ in mitochondria

probe $23 .{ }^{49}$ In this BODIPY based probe, TPP was introduced as the targeting group and an $N$-benzyl-4-hydroxyaniline moiety was the reaction site. The PET process in $\mathbf{2 3}$ was disrupted when the fast and specific N-nitrosation of the aromatic secondary amine occurred, resulting in a 92 -fold fluorescence enhancement at $518 \mathrm{~nm}$. The LOD of 23 was estimated to be $4.8 \mathrm{nM}$. With probe 23, the NO levels in macrophages in the resting state or under diverse stimulations were successfully visualized.

4.2.2. Imaging of $\mathrm{ONOO}^{-}$in mitochondria. $\mathrm{ONOO}^{-}$can be generated from the reaction between the $\mathrm{NO}$ and $\mathrm{O}_{2}{ }^{-}{ }^{-}$. $\mathrm{ONOO}^{-}$ has high reactivity and excellent membrane permeability, which makes it an ideal intracellular regulator toward diverse biomacromolecules. ${ }^{45,46,78}$ It has been reported that $\mathrm{ONOO}^{-}$is involved in the development of numerous major diseases, such as cardiovascular disease, neurodegenerative disease, acute inflammation and cancer. ${ }^{44,47,79}$ Therefore, fluorescent probes for tracing the subcellular $\mathrm{ONOO}^{-}$levels during different processes are of significant importance. Oxidation of phenylhydrazine and unsaturated bonds, deboronation and decomposition of $\alpha$-ketoamide groups are the most popular mechanisms for subcellular localized $\mathrm{ONOO}^{-}$probe design (Fig. 14).

Qian and co-workers devised a ratiometric probe, $\mathbf{2 4}$ (PNCy3Cy5), for mitochondrial endogenous $\mathrm{ONOO}^{-}$imaging. ${ }^{45}$ 24 was prepared by covalent connection of the famous FRET pair, Cy3 and Cy5, through a $1.4 \mathrm{~nm}$ linker. In this probe, Cy5 could be readily oxidized by $\mathrm{ONOO}^{-}$, while $\mathrm{Cy} 3$ was stable due to the shorter polymethine chain. Under $530 \mathrm{~nm}$ laser excitation, 24 emitted fluorescence at $660 \mathrm{~nm}$ (belonging to Cy5) due to the FRET effect. Upon the addition of $\mathrm{ONOO}^{-}$to the probe solution, the fluorescence intensity at $660 \mathrm{~nm}$ decreased due to the oxidation of Cy5 and the emission band at $560 \mathrm{~nm}$ emerged as a result of disruption of FRET. Confocal imaging experiments revealed the excellent cellular permeability and mitochondriontargeting capability of this probe, and the LOD of $\mathbf{2 4}$ was determined to be $0.65 \mathrm{nM}$. Endogenous $\mathrm{ONOO}^{-}$in macrophage cells under diverse stimulations was monitored in a ratiometric imaging manner, which suggested that $\mathbf{2 4}$ was promising for revealing the biological and pathological functions of $\mathrm{ONOO}^{-}$.

Yuan and co-workers reported TP probe 25 (MITO-CC) for mitochondrial $\mathrm{ONOO}^{-}$imaging. ${ }^{78} 19$ fluorescent compounds were screened under physiological conditions, and they obtained a mitochondrion-specific fluorophore with high sensitivity and selectivity toward $\mathrm{ONOO}^{-}$. By further connecting coumarin as the energy donor, a mitochondrion-targeted ratiometric probe with high fluorescence quantum yield, high molar absorbance, excellent photostability and solubility was obtained. 25 has a fast response rate (less than $20 \mathrm{~s}$ ) and low LOD $\left(1.13 \times 10^{-8} \mathrm{M}\right)$ toward $\mathrm{ONOO}^{-}$. Ratiometric imaging of endogenous mitochondrial $\mathrm{ONOO}^{-}$differences between normal and inflamed cells or tissues was realized with this probe (Fig. 15), and it suggested that 25 was favourable for tracing the small fluctuations of $\mathrm{ONOO}^{-}$in diverse biological processes.

DILI is a common threat for public health. It has been reported that $\mathrm{ONOO}^{-}$could be an important DILI indicator, so imaging of the $\mathrm{ONOO}^{-}$levels in living cells can be critical for drug toxicity testing. Yuan et al. further developed 26 (Rhod- 

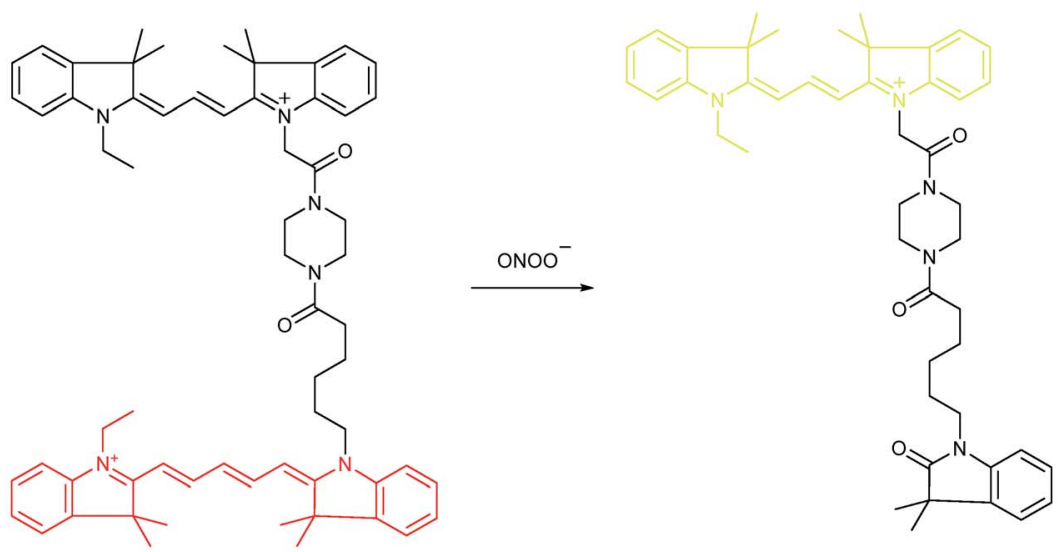

$24\left(\mathrm{PNC}_{3} \mathrm{Cy}_{5}\right)$<smiles></smiles><smiles></smiles><smiles></smiles>

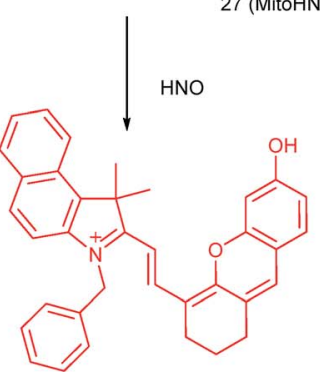

26 (Rhod-ONOO ${ }^{-}$)

Fig. 14 Fluorescent probes for imaging $\mathrm{ONOO}^{-}$and $\mathrm{HNO}$ in mitochondria.

$\mathrm{ONOO}^{-}$) by a combination of ICT and PET mechanisms, for mitochondrion-targeted $\mathrm{ONOO}^{-}$detection. ${ }^{47}$ Confocal imaging experiments in HepG2 cells revealed the excellent mitochondrion-targeting capacity of $\mathbf{2 6}$. Mitochondrial $\mathrm{ONOO}^{-}$ generation during DILI was visualized with this probe, and the drug-induced hepatotoxicity pathway was successfully discriminated. Therefore, $\mathbf{2 6}$ is a powerful tool for DILI measurement and it also proved the great promise of subcellular localized fluorescent probes in bioanalysis and drug discovery.

4.2.3. Imaging of $\mathbf{H N O}$ in mitochondria. $\mathrm{HNO}$ can be generated from $N$-hydroxy-L-arginine via catalysis by $\mathrm{NO}^{\circ}$

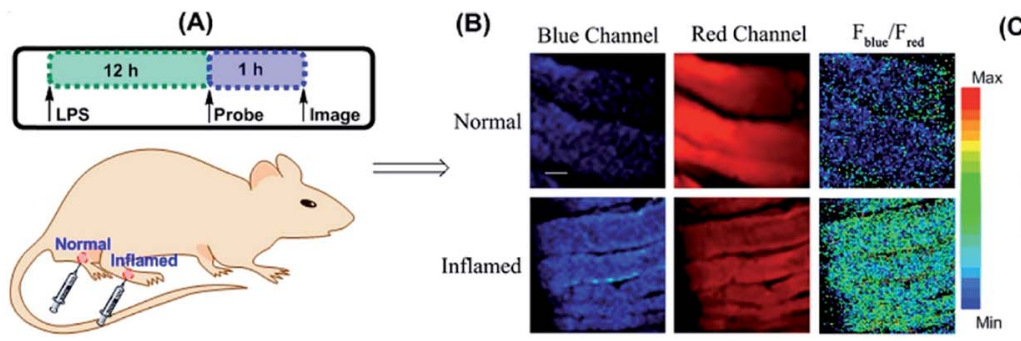

(C)

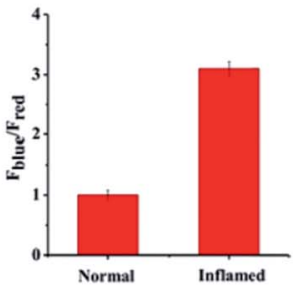

Fig. 15 TP fluorescence imaging of $\mathrm{ONOO}^{-}$generation in inflamed tissues. (A) $200 \mu \mathrm{L}$ of LPS (1 mg mL $\left.{ }^{-1}\right)$ was subcutaneously injected into the right leg of mice to cause inflammation. After 12 h, $20 \mu \mathrm{L}$ of $500 \mu \mathrm{M} 25$ was subcutaneously injected in situ. After $1 \mathrm{~h}$, the leg skin of mice was sectioned after being anaesthetized. (B) Fluorescence images of 25 in the normal and inflamed tissues. (C) Average $F_{\text {blue }} / F_{\text {red }}$ intensity ratios in panel (B). Blue channel, $\lambda_{E m}=460-500 \mathrm{~nm}$; red channel, $\lambda_{E m}=605-680 \mathrm{~nm}$. $\lambda_{E x}=800 \mathrm{~nm}$. Adapted with permission from ref. 78 . Copyright 2016 American Chemical Society. 
synthase. ${ }^{50 b, 80}$ HNO exerts numerous biological functions in organisms. For instance, it can adjust diverse signal channels, inhibit the activity of diverse enzymes and prevent platelet aggregation. The Staudinger reaction is the most popular mechanism for bio-orthogonal HNO probes. ${ }^{\mathbf{1}}$

In 2016, Yang et al. reported 27 (MitoHNO) as the first mitochondrion-targetable NIR HNO probe. ${ }^{81 a}$ A 2-(diphenylphosphino)benzoyl group was employed as the HNO recognition group, and a lipophilic indolium cation was used as the targeting moiety. During the reaction between 27 and HNO, 38fold fluorescence intensity enhancement at $727 \mathrm{~nm}$ was observed due to the release of a fluorophore. The LOD of 27 was estimated to be $60 \mathrm{nM}$. Mitochondrial HNO in SMMC-7721 cells was specifically detected with this probe.

\subsection{Imaging of RSS in mitochondria}

As discussed above, mitochondria perform numerous respiration reactions to maintain cellular ATP synthesis, and this makes the mitochondrial ROS and RNS levels higher than those in other subcellular compartments. Reductive species are indispensable for signal transduction and protecting mitochondria from oxidative stress; therefore, they are also reliable biomarkers for diverse bioactivities as well as diseases. ${ }^{\mathbf{5 6 , 5 8 , 5 9 , 8 2}}$ Fluorescent probes for tracing the localized RSS concentration in mitochondria hold great promise for advancing our understanding of the physiological and pathological functions of RSS, which should also be beneficial for the exploration of novel drugs toward diverse related diseases (Fig. 16).

4.3.1. Imaging of GSH in mitochondria. Intracellular GSH has drawn increasing interest since it is one of the most abundant biothiols in living cells, and acts as an antioxidant reservoir to mitochondria and a biomarker of oxidative stress. Several probes have been reported for mitochondrial GSH imaging, based on either the Michael addition or nucleophilic substitution reaction.

Kim's group reported a mitochondrion-targeted NIR probe, 28 (MitoGP), for GSH imaging. ${ }^{52} \mathrm{~A}$ heptamethine unit was used as the fluorophore owing to its NIR emissive and mitochondrion-targeting capacity, while a nitroazo group was employed as the GSH recognition group and fluorescence quencher. Significant fluorescence enhancement at $810 \mathrm{~nm}$ was observed in the presence of GSH, due to the specific 1,6conjugate addition and subsequent elimination reaction triggered fluorophore release. 28 exhibited high selectivity toward GSH over other biothiols, and the LOD was estimated to be 10 $\mu \mathrm{M}$. The mitochondrion-targeting capability of $\mathbf{2 8}$ was demonstrated to be comparable to that of the commercialized MitoTracker, and mitochondrial GSH in HeLa cells under different stimulations was easily monitored with this probe (Fig. 17).

Zhao et al. reported a BODIPY based probe (29) for monitoring mitochondrial GSH. ${ }^{83}$ The self-immolative group paradinitrophenoxy benzyl pyridinium was introduced at the meso position of BODIPY, and it could simultaneously act as the targeting ligand and responsive group. Upon reaction with $\mathrm{GSH}$, the dinitrophenyl moiety of 29 was first cleaved through an $\mathrm{S}_{\mathrm{N}} \mathrm{Ar}$ reaction; subsequently, the fluorophore BODIPY was released via a self-immolation reaction, which results a 32 -fold fluorescence intensity enhancement at $599 \mathrm{~nm}$. 29 was used for mitochondrion-targeted GSH imaging in HeLa cells.

Compared to traditional fluorescent probes, the design of molecular tools for bioactive species imaging based on a reversible reaction is still a challenge. However, reversible probes are promising for elucidating the dynamic states of analytes of interest in different processes, and would offer more information in cell imaging. Wang et al. introduced the first mitochondrion-targeted probe, 30 (MitoRT), for the reversible detection of GSH. ${ }^{51}$ TPP was linked with the fluorophore through the optimized 4-carbon linker. Benefiting from the reversibility, 30 responds to both increases and decreases of GSH levels and can be used for ratiometric imaging. The response time of this probe was less than $30 \mathrm{~s}$. 30 has been successfully used to monitor mitochondrial GSH levels during redox perturbance. Importantly, with this probe, it was demonstrated that cells prioritize to maintain mitochondrial GSH levels during cell growth.

4.3.2. Imaging of Cys in mitochondria. Cys is an important amino acid for protein fabrication, and it plays critical roles in protein disulfide bond formation during protein cross-linking and folding. ${ }^{53 b, c, 84}$ Moreover, Cys has the potential to coordinate with metal ions in living cells, and thereby to regulate their intracellular concentration and avoid toxicity effects caused by excess heavy metal ions. The abnormal Cys concentration in living cells is closely related to diverse diseases, including rheumatic arthritis, Alzheimer's disease, Parkinsonism and so on. ${ }^{53 c, 84 a}$ Tracing subcellular Cys concentration fluctuation has drawn research interest due to its potential in facilitating healthcare.

$\mathrm{Li}$ and Feng et al. reported a semiheptamethine derivative, 31 (NFL1), for selective detection of Cys in mitochondria. ${ }^{53 b}$ Based on the specific Michael addition and subsequent cyclization reaction, strong NIR emission intensity enhancement at $735 \mathrm{~nm}$ was observed in the presence of Cys, as a result of the disruption of the PET process. The LOD of $\mathbf{3 1}$ was estimated to be as low as $14.5 \mathrm{nM} .31$ has been successfully applied for the assessment of mitochondrial oxidative stress. Cys imaging in a living nude mice model was also demonstrated owing to its NIR emissive features (Fig. 18).

4.3.3. Imaging of $\mathrm{H}_{2} \mathrm{~S}$ in mitochondria. $\mathrm{H}_{2} \mathrm{~S}$, as the simplest biothiol, has been reported to be another important gasotransmitter in living cells after NO and CO. ${ }^{56}$ Endogenous $\mathrm{H}_{2} \mathrm{~S}$ can be synthesized by several enzymes in living cells. Research studies have suggested that $\mathrm{H}_{2} \mathrm{~S}$ can regulate many physiological processes, such as vascular tension, myocardial preservation, angiogenesis and mitochondrial powering. ${ }^{\mathbf{1 2 c}, \mathbf{2 6} b}$ The unregulated expression of $\mathrm{H}_{2} \mathrm{~S}$ is often involved in numerous major diseases, like cancer, Alzheimer's diseases and diabetes. In the past decade, a series of probes have been developed for subcellular $\mathrm{H}_{2} \mathrm{~S}$ detection. The detection mechanisms of these probes mainly include the reduction of aryl azide and nitro groups, nucleophilic addition reaction, precipitation of $\mathrm{Cu}^{2+}$, and disulfide exchanges. ${ }^{3 c}$

Yoon et al. reported a naphthalimide-based probe (32) with the 7-nitro-1,2,3-benzoxadiazole (NBD) moiety for mitochondrial $\mathrm{H}_{2} \mathrm{~S}$ detection. ${ }^{85}$ The fluorescence of 32 was dark due to the 

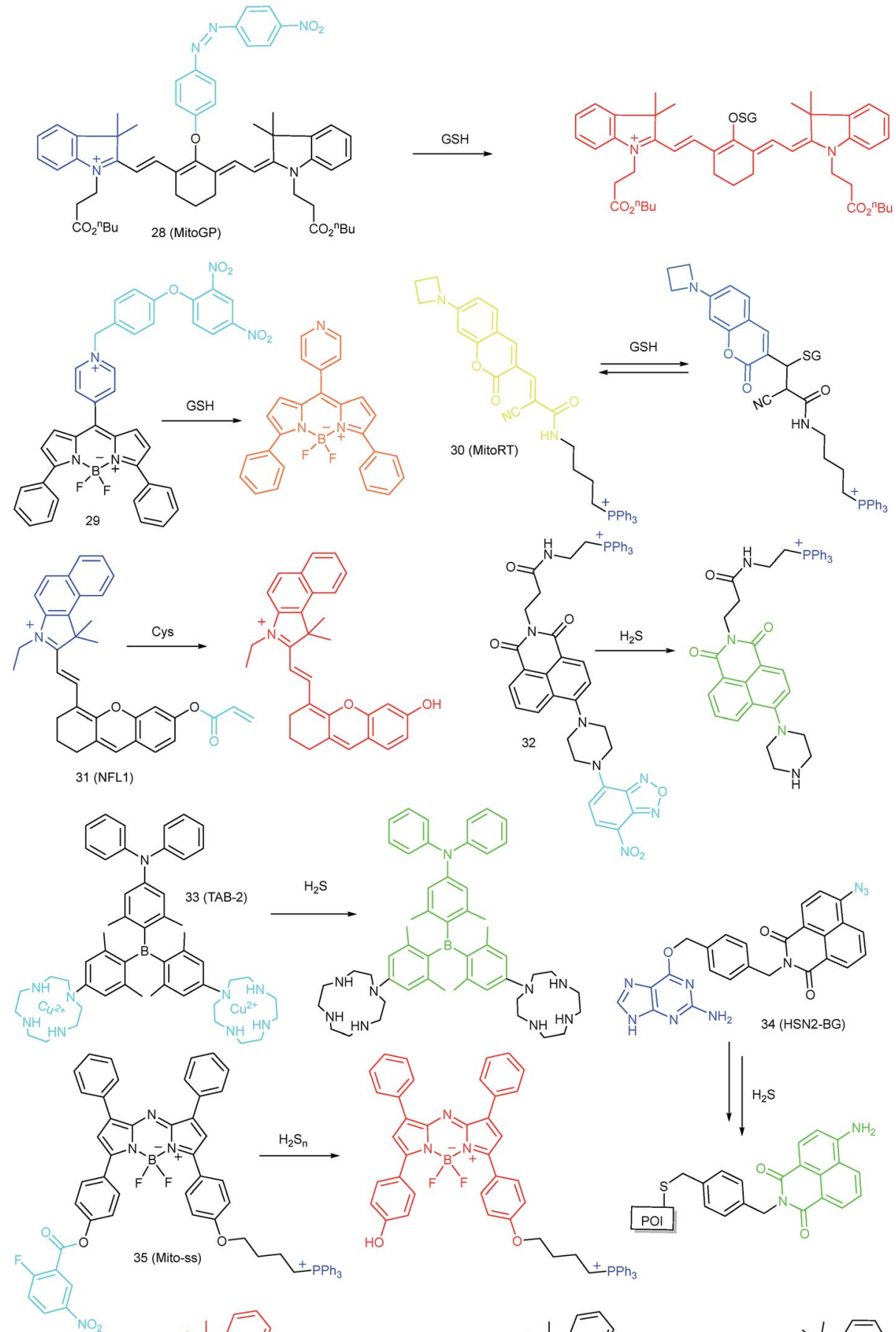

$\mathrm{C}_{\mathrm{NO}_{2}}^{\mathrm{N}}$<smiles></smiles>
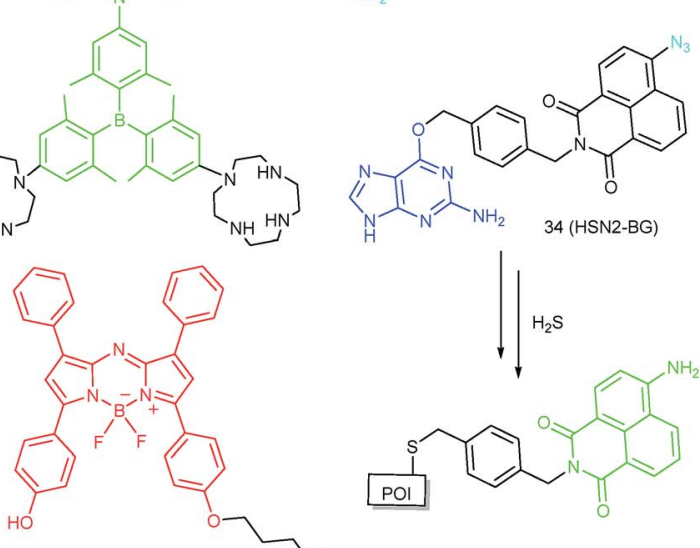

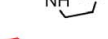
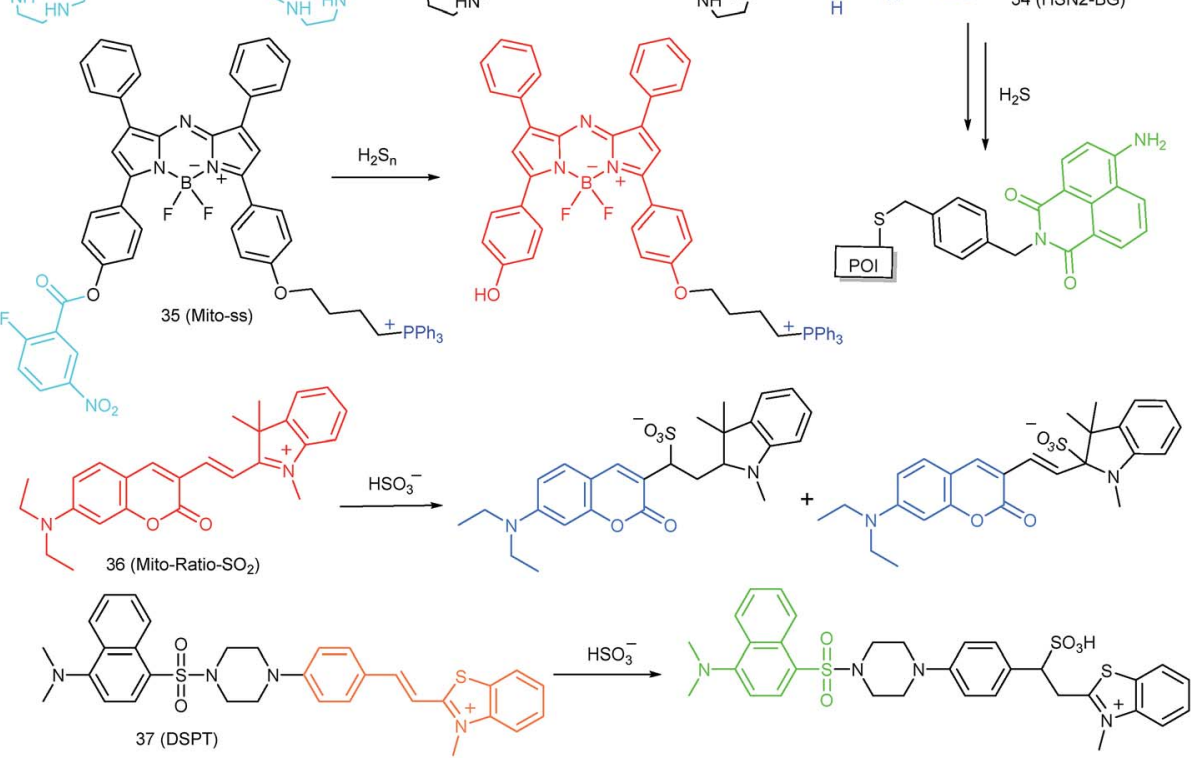

Fig. 16 Fluorescent probes for imaging RSS in mitochondria. 

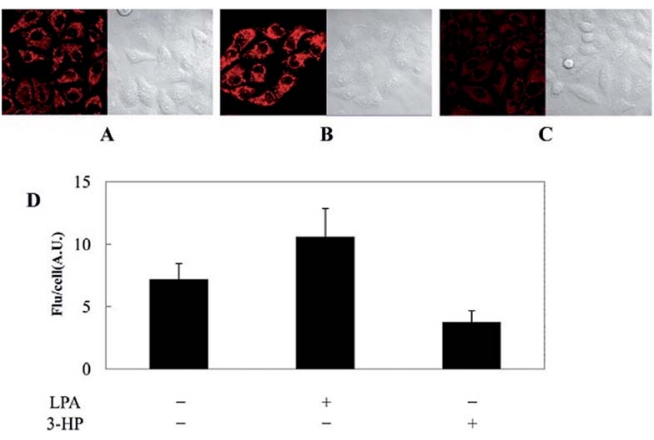

Fig. 17 CLSM images of HeLa cells subjected to different treatments (A-C) with 28. (A) 28 only, (B) 28 + LPA (1.8 mM, 24 h), and (C) $28+3-$ HP (1 mM, 5 min then $2 \mathrm{~h}$ incubation); (D) their histograms of fluorescence intensities. Adapted with permission from ref. 52 . Copyright 2014 American Chemical Society.
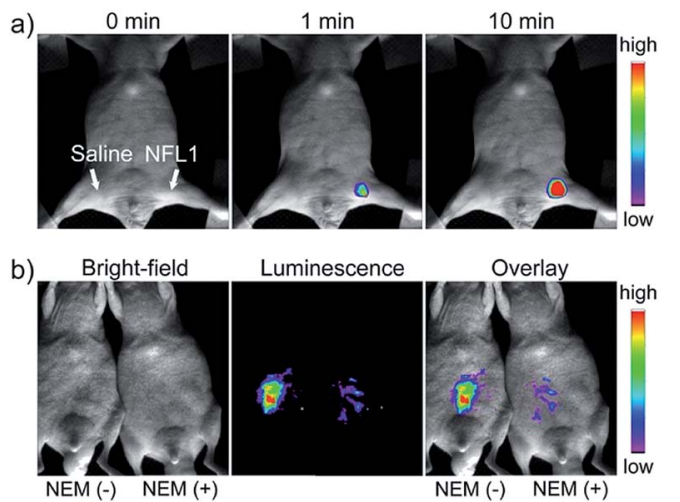

Fig. 18 In vivo NIR imaging of living mice subjected to different treatments with probe 47. (a) The mice was treated with $0.1 \mathrm{~mL}$ of saline (left side) or $31(10 \mu \mathrm{M}$, right side) solution and imaged at different times after subcutaneous injection of 47. (b) The left mice was injected with probe $31(10 \mu \mathrm{M}, 0.2 \mathrm{~mL})$ only for $10 \mathrm{~min}$. The right mice was preinjected with NEM ( $1 \mathrm{mM}, 0.2 \mathrm{~mL})$ for $30 \mathrm{~min}$ and then injected with $31(10 \mu \mathrm{M}, 0.2 \mathrm{~mL})$ for $10 \mathrm{~min}$. Adapted with permission from ref. 53b. Copyright 2015 American Chemical Society.

quenching effect of the NBD moiety, but it underwent 68-fold intensity enhancement at $532 \mathrm{~nm}$ in the presence of $\mathrm{Na}_{2} \mathrm{~S}$ as a consequence of the cleavage of the NBD amine and release of a fluorophore. Confocal imaging experiments revealed that the probe localized in mitochondria well. The mitochondrial $\mathrm{H}_{2} \mathrm{~S}$ of HeLa cells under different treatments could be monitored with this biocompatible probe.

Yang et al. devised a triarylboron based "turn-on" probe, 33 (TAB-2), for mitochondrial $\mathrm{H}_{2} \mathrm{~S}$ imaging. ${ }^{56}$ The probe was prepared by functionalization of one diphenylamine and two cyclens at the para position of phenyl groups of triarylboron. After complex formation with $\mathrm{Cu}^{2+}$, the fluorescence of TAB-2 was quenched due to the inhibition of the ICT process. Upon exposure to $\mathrm{H}_{2} \mathrm{~S}$, dramatic fluorescence intensity recovery was observed; the LOD of 33 was $47 \mathrm{nM}$. Interestingly, the lipophilic cationic structure endowed $\mathbf{3 3}$ with the capability of mitochondrion-targeting. 33 has been employed for TP and fluorescence lifetime imaging of $\mathrm{H}_{2} \mathrm{~S}$ in NIH/3T3 cells.
Taking advantage of the SNAP-tag technology, Pluth's group reported a probe, 34 ( $\mathrm{HSN} 2-\mathrm{BG}$ ), for discriminating the $\mathrm{H}_{2} \mathrm{~S}$ release profiles of different $\mathrm{H}_{2} \mathrm{~S}$ donor molecules. ${ }^{12 b}$ In view of the existence of $\mathrm{H}_{2} \mathrm{~S}$ synthase in mitochondria, and some that the $\mathrm{H}_{2} \mathrm{~S}$ donor can store in the lysosomes, they transfected and expressed AGT protein into mitochondria and lysosomes, respectively. Benefiting from the precise subcellular localization of the probes, they revealed the disparate $\mathrm{H}_{2} \mathrm{~S}$ release profiles between different $\mathrm{H}_{2} \mathrm{~S}$ donors in mitochondria and lysosomes, offering a new fluorescent tool for the investigation of subcellular $\mathrm{H}_{2} \mathrm{~S}$.

4.3.4. Imaging of $\mathbf{H}_{2} \mathbf{S}_{n}$ in mitochondria. Chen et al. developed a bis-electrophilic group containing probe, 35 (Mito-ss), for mitochondrial $\mathrm{H}_{2} \mathrm{~S}_{n}$ detection. ${ }^{58}$ TPP was employed as the targeting ligand to endow the probe with mitochondriontargeting capability. Nitro-activated fluorobenzoiate was introduced into the fluorophore, acting as the fluorescence quencher and $\mathrm{H}_{2} \mathrm{~S}_{n}$ recognition site. In the presence of $\mathrm{H}_{2} \mathrm{~S}_{2}, 35$ underwent a nucleophilic aromatic substitution reaction, and formed a -SH containing intermediate. Subsequently, an intramolecular cyclization took place. Thereby the PET process of 35 was blocked, and 24-fold fluorescence enhancement was observed during this process. Based on the two-step reaction, this probe can avoid interference from thiols. The reaction could be finished in $30 \mathrm{~s}$, and the LOD of 35 was $25 \mathrm{nM}$. The process of the conversion of $\mathrm{H}_{2} \mathrm{~S}$ into $\mathrm{H}_{2} \mathrm{~S}_{n}$ to eliminate the intracellular ROS directed by glutathione peroxidase was confirmed with this probe. In vivo visualization of $\mathrm{H}_{2} \mathrm{~S}_{n}$ was also carried out owing to the NIR emissive merit of 35.

4.3.5. Imaging of $\mathrm{SO}_{2}$ in mitochondria. $\mathrm{SO}_{2}$ is a key air pollutant involved in the formation of acid rain, and can cause diverse side effects in the human body. $\mathrm{SO}_{2}$ can also be synthesized in living cells by the oxidation of sulfur-containing molecules including amino acids. ${ }^{\mathbf{1 2 a}, 59}$ Abnormal cell metabolism may result in the disruption of homeostasis of $\mathrm{SO}_{2}$ in living cells. An increasing number of reports have proved that $\mathrm{SO}_{2}$ is involved in a series of pathological processes, including lung cancer, and urticarial and gastrointestinal disorders. ${ }^{86}$ For in situ and real-time visualization of $\mathrm{SO}_{2}$ in living cells, a variety of probes have been designed in recent years. Among them, mitochondrion-targeted ones are quite attractive.

In 2015, Yuan and Chang et al. reported the first example of a mitochondrion-targeted ratio probe, 36 (Mito-Ratio- $\left.\mathrm{SO}_{2}\right){ }^{59 a} \mathrm{~A}$ mechanism study revealed that the nucleophilic addition of $\mathrm{SO}_{2}$ to 36 could generate two stable adducts and block the ICT process within the probe. The blocked ICT process between coumarin and indole groups resulted in a blue-shifted emission. Interestingly, the emission shift between the two bands was as large as $170 \mathrm{~nm} .36$ had high selectivity toward $\mathrm{SO}_{2}$ with a LOD of $0.09 \mu \mathrm{M}$. The generation of $\mathrm{SO}_{2}$ in the mitochondria of HeLa cells was detected with this ratiometric probe.

Zhao's group devised probe 37 (DSPT) based on the FRET mechanism, and it was constructed from the dansyl-piperazinebenzothiazolium conjugate platform. ${ }^{87}$ In this probe, the cationic benzothiazolium group was employed as the targeting group, while a neighbouring $\mathrm{C}=\mathrm{C}$ bond was the recognition moiety. The FRET process of the probe was "on" due to the 
spectral overlap, but was "switched off" in the presence of $\mathrm{HSO}_{3}{ }^{-}$, as a consequence of the fact that the Michael addition reaction disrupted the conjugated structure of the acceptor fluorophore. The LOD of 37 was $69 \mathrm{nM}$, which indicates that it was suitable for detecting $\mathrm{SO}_{2}$ derivatives in diverse real samples. The exogenous and endogenous $\mathrm{SO}_{2}$ derivatives in the mitochondria of HepG2 cells were visualized in a ratiometric imaging manner.

\subsection{Challenges for mitochondrion-targeted probes}

Benefiting from the critical biological function and high activity of mitochondria, fluorescent probes, taking mitochondria as the targeting sites, have been widely developed and employed for RONSS imaging in different cases. However, the majority of targeting ligands are lipophilic cationic structures that carry the risk of reducing MMP. Also, during diverse stimulations or fixation with chemical agents, these dyes can easily leak out due to the MMP lost, and cannot work in these situations. Genetically encoded probes are capable of covalently anchoring to mitochondria with high selectivity and may avoid the dye leakage. But the gene transfection efficiency cannot be guaranteed, while repeated washing may change the mitochondrial microenvironment. Also, the gene transfecting process makes the probes difficult to use for in vivo imaging (imaging mitochondria during liver/kidney IRI and so on). Moreover, there are hundreds of biomolecules in mitochondria, including enzymes, cations, anions, lipids, mitochondrial DNA, RNA and so on. In situ selective staining of bioactive molecules at ultralow concentrations (usually nanomolar amounts) using fluorescent probes is quite challenging. Many reported probes only work when large amounts of exogenous analytes are added or cells are stimulated by diverse chemical agents. Probes capable of selectively imaging bioactive species at basal concentrations are still rare, which hampers the further explorations of the physiological functions of these species. Ratiometric probes are promising for eliminating diverse interferences, but only limited mitochondrion-targeted ratiometric probes have been designed and constructed up to now.

\section{Lysosome-targeted probes}

As the main digestive compartments of cells, lysosomes store numerous enzymes for degradation/recycling of diverse exogenous and endogenous biomolecules, and can effectively defend cells from infection, starvation and stress. ${ }^{3 b, 40 a, b, 88}$ The bioactivity of RONSS makes them feasible for regulating the enzyme activity in such organelle, and serious disorders can be caused when expressed in an uncontrolled manner. As people have gradually recognized the importance of lysosomes, more and more lysosome-targeted fluorescent probes have been explored for RONSS imaging in different study models.

\subsection{Imaging of ROS in lysosomes}

Benefiting from the acidic and digestive lysosomal microenvironments, the activities of diverse ROS scavenging enzymes (such as SOD and peroxidase) in lysosomes are restricted, which facilitates the ROS accumulation in such single-layered membrane bounded organelle. ${ }^{28,89}$ The lysosomal ROS level is closely related to the lysosomal enzyme regulation, which can be critical for maintaining cellular homeostasis. Lysosomal oxidative stress caused by over-accumulation of ROS can result in lipid peroxidation, which will further cause lysosomal membrane permeabilization (LMP) and cell disorders. ${ }^{90}$ Therefore, monitoring the lysosomal ROS concentration has been recognized as an important task for molecular pathology. Novel fluorescent probes capable of tracing the lysosomal ROS fluctuation are highly significant for bioanalysis (Fig. 19).

5.1.1. Imaging of $\mathrm{H}_{2} \mathrm{O}_{2}$ in lysosomes. Lin et al. developed a lysosome-targeted naphthalimide based $\mathrm{TP} \mathrm{H}_{2} \mathrm{O}_{2}$ probe, 38 (Lyso-HP). ${ }^{91}$ Morpholine was employed as the lysosometargeting group, while the electron-deficient boronate group was used as the recognition site. The "A- $\pi-\mathrm{A}$ " structure made 38 emit weakly. When the boronate group of $\mathbf{3 8}$ was specifically oxidized to hydroxyl, a "D- $\pi-\mathrm{A}$ " electronic structure was formed, and the re-activated ICT process resulted in significant fluorescence "turn-on". TP imaging experiments in living cells and tissues demonstrated that $\mathbf{3 8}$ could act as a novel candidate for lysosomal $\mathrm{H}_{2} \mathrm{O}_{2}$ detection.

Kumar et al. reported another morpholine decorated naphthalimide based probe, 39 (LyNC), for lysosomal $\mathrm{H}_{2} \mathrm{O}_{2}$ imaging, and it employed catechol as the reaction site. ${ }^{37}$ In the absence of $\mathrm{H}_{2} \mathrm{O}_{2}$, the PET process between the catechol moiety and naphthalimide blocked the fluorescence; in the presence of $\mathrm{H}_{2} \mathrm{O}_{2}$, the probe emitted evidently due to the disruption of the PET process caused by oxidation of catechol into $o$-quinone. 39 was successfully used for tracing the lysosomal $\mathrm{H}_{2} \mathrm{O}_{2}$ in $\mathrm{C} 6$ glioma cells, BV-2 microglial cells and nematodes under different stimulations.

In view of the acidic lysosomal microenvironments, probes that can simultaneously respond to the $\mathrm{pH}$ and $\mathrm{H}_{2} \mathrm{O}_{2}$ should be promising for reducing the background during fluorescent imaging. Wu and Zhao et al. introduced an acidic $\mathrm{pH}$ activatable probe, 40 (HP-L1), for lysosomal $\mathrm{H}_{2} \mathrm{O}_{2}$ detection. ${ }^{28}$ In this probe, boronate was employed as the responsive moiety, and spirobenzopyran as the $\mathrm{pH}$ switchable fluorophore. This probe could only emit intensive fluorescence in lysosomes after reacting with $\mathrm{H}_{2} \mathrm{O}_{2}$, which made it a powerful tool for avoiding the background fluorescence during lysosomal $\mathrm{H}_{2} \mathrm{O}_{2}$ imaging.

5.1.2. Imaging of $\mathbf{H O C l}$ in lysosomes. HOCl plays critical roles in cell immune systems and is closely related to diverse pathological processes. ${ }^{\mathbf{4 0}, \mathbf{8 8 , 9 2}}$ Development of lysosomaltargeted probes for $\mathrm{HOCl}$ has attracted increasing interest in recent years. As discussed above, the detection mechanisms of fluorescent probes toward $\mathrm{HOCl}$ mainly rely on the oxidation capacity of HOCl.

In 2015, Chang's group reported an "off-on” HOCl probe, 41 (TP-HOCl 1), with a fast response rate (within seconds) and low LOD $(16.6 \mathrm{nM}) .^{40 b}$ The response mechanism of 41 was the $\mathrm{HOCl}$ mediated oxidative deprotection of the oxathiolane/mercaptal group, which resulted in the recovery of the "push-pull" structure in acedan and production of fluorescence at $500 \mathrm{~nm} . \mathbf{4 1}$ exhibited remarkable specificity toward $\mathrm{HOCl}$ among diverse ROS. Further modification of morpholine or TPP with 41 

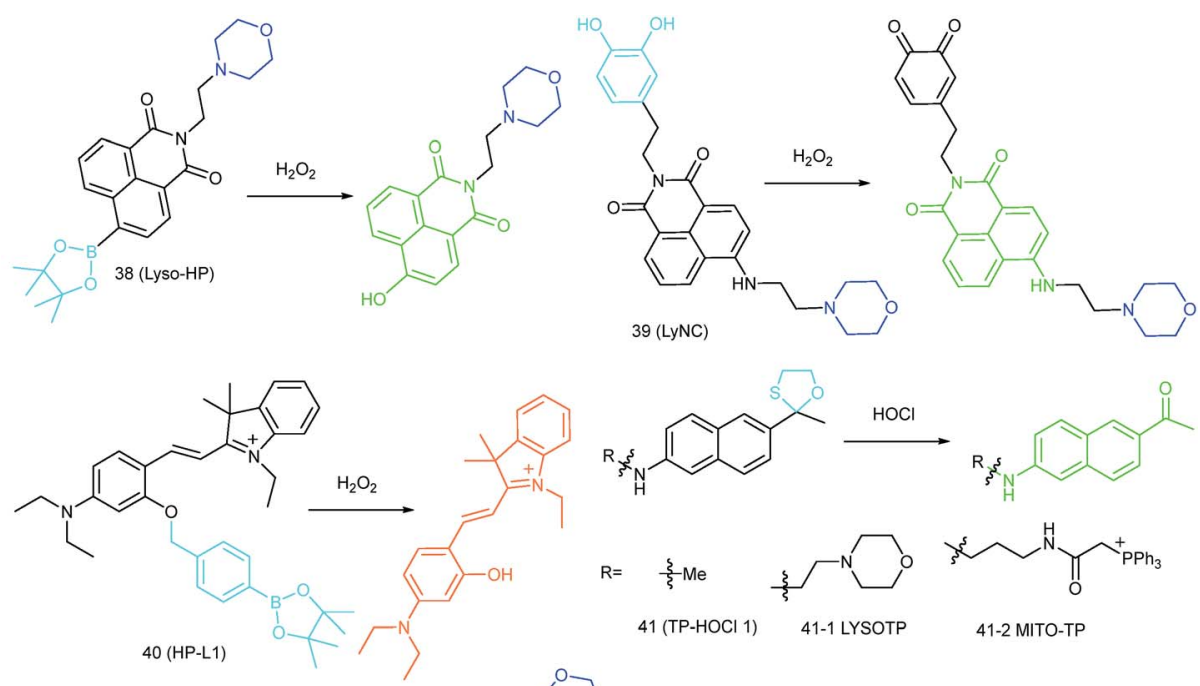

41 (TP-HOCI 1) 41-1 LYSOTP

41-2 MITO-TP
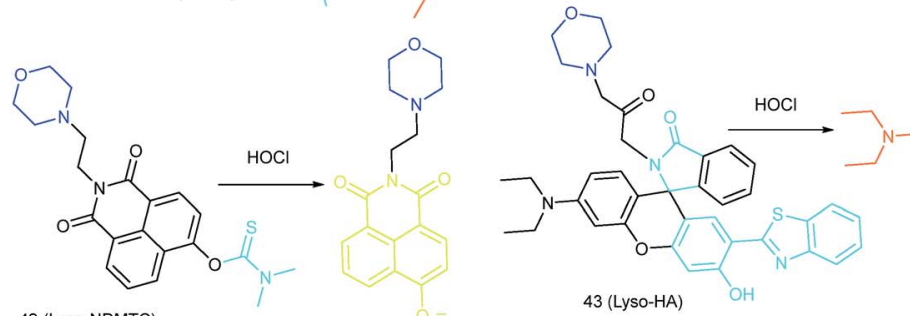

42 (Lyso-NDMTC)

43 (Lyso-HA)

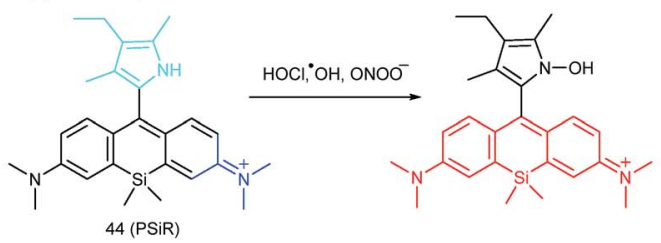

Fig. 19 Fluorescent probes for imaging ROS in mitochondria.

yielded lysosome- and mitochondrion-targeted probes (41-1 LYSOTP and 41-2 MITO-TP, respectively). They were successfully employed for TP imaging under both in vitro and in vivo conditions, and the subcellular distribution of $\mathrm{HOCl}$ was elucidated.

Our group prepared a thiocarbamate derivative based TP probe, 42 (Lyso-NDMTC), for lysosomal HOCl imaging. ${ }^{88}$ This probe was fabricated by decorating naphthalimide with morpholine and $N, N$-dimethylthiocarbamate (DMTC). 42 emitted faintly initially but highly after reacting with the analyte of interest, due to the electrophilic addition reaction of $\mathrm{Cl}^{+}$ induced cleavage of DMTC. Benefiting from the reaction reactivity of DMTC and sensitivity of the ICT process, the LOD of $\mathbf{4 2}$ was estimated to be $7.6 \mathrm{pM} .42$ is the first probe capable of imaging the basal lysosomal $\mathrm{HOCl}$ at the picomolar level, and it has been successfully employed to distinguish tumor tissue from normal tissue in vivo with the assistance of TP microscopy (Fig. 20).

Lin's group devised a lysosome-targeted ratiometric $\mathrm{HOCl}$ probe, 43 (Lyso-HA), based on the ESIPT mechanism..$^{92}$ Before coming into contact with $\mathrm{HOCl}, \mathbf{4 3}$ adopted the spirocyclic form and the probe underwent the ESIPT process between the phenolic hydroxyl group and benzazole ring, which gave

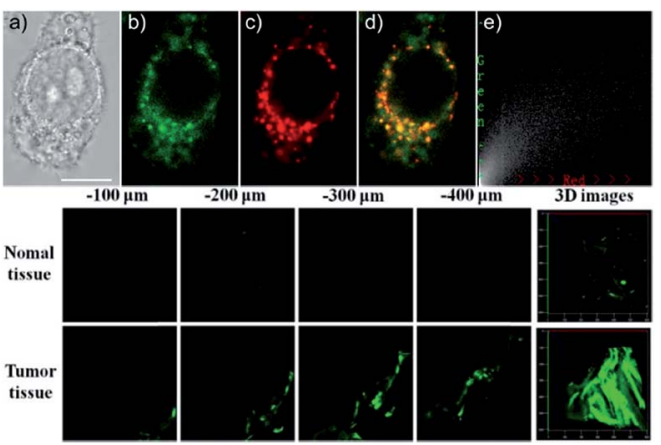

Fig. 20 Top: CLSM images of live HepG2 cells pretreated with 42 $(20.0 \mu \mathrm{M})$ and Lyso-Tracker Red (100.0 nM) for $20 \mathrm{~min}$ : (a) bright-field image, (b) green channel for probe fluorescence, (c) red channel for Lyso-Tracker fluorescence, (d) merged image from panels (b) and (c); and (e) intensity correlation plot of $\mathbf{4 2}$ and Lyso-Tracker Red. Bottom: Two-photon fluorescence imaging at different depths and the 3D distribution of $\mathrm{HOCl}$ in the normal and cancerous breasts of mice pretreated with $42(100.0 \mu \mathrm{M})$ for $30 \mathrm{~min}$. Fluorescence images were obtained with an $800 \mathrm{~nm}$ light source. Adapted with permission from ref. 88. Copyright 2016 American Chemical Society. 
emission at $450 \mathrm{~nm}$. Upon reaction with $\mathrm{HOCl}$, the spirocyclic ring opened and there was no proton close to the benzazole ring. The blocking of the ESIPT process resulted in a decrease in the emission peak at $450 \mathrm{~nm}$ as well as the appearance of a new emission band at $586 \mathrm{~nm}$. Benefiting from the remarkable emission wavelength red shift (136 nm), 43 is an ideal tool for exogenous and endogenous lysosomal HOCl imaging (Fig. 21).

5.1.3. Imaging of hROS in lysosomes. Highly reactive oxygen species (hROS, including $\mathrm{ClO}^{-},{ }^{\cdot} \mathrm{HO}$ and so on) are closely related to oxidative stress due to their reactivity and they are overexpressed in diverse diseased cells. Novel probes for monitoring hROS in subcellular microenvironments are promising for disease diagnosis. In 2017, Guo et al. reported a lysosome-targeted Si-rhodamine-based probe, 44 (PSiR), for hROS imaging. ${ }^{89} 44$ employed Si-rhodamine dye as the fluorophore, and 2,4-dimethyl-3-ethylpyrrole as the electron donor and responsive site. $\mathbf{4 4}$ was non-emissive due to the intramolecular PET process. Upon reaction with hROS $\left(\mathrm{ClO}^{-}, \mathrm{ONOO}^{-}\right.$, and $\left.{ }^{\circ} \mathrm{HO}\right)$, 180-fold fluorescence enhancement at $680 \mathrm{~nm}$ was observed from the solution of 44. Endogenous hROS in the lysosomes of A549 cells after chemotherapeutic drug stimulation was observed. Moreover, cancer cells and tissues (Fig. 22)

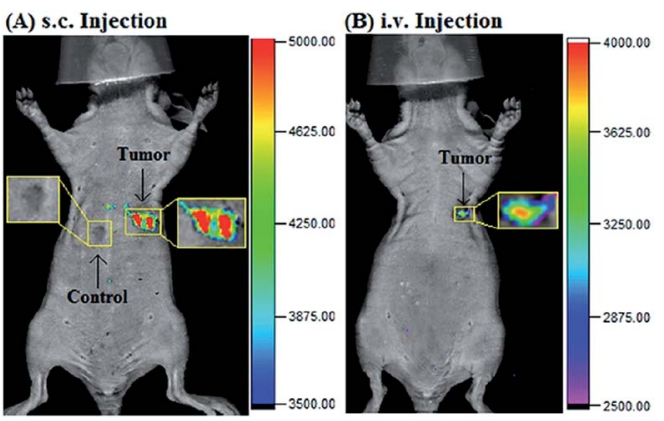

Fig. 22 Imaging tumor using 44 in the tumor-bearing mouse xenograft models prepared by subcutaneous inoculation of HeLa cells. (A) The mouse was subcutaneously injected with $44(2 \mathrm{mM}, 10 \mu \mathrm{L})$ in the tumor region and normal region, respectively, and then imaged immediately. (B) The mouse was intravenously injected with $44(1.5 \mathrm{mg}$ $\left.\mathrm{kg}^{-1}\right)$, and then imaged after $25 \mathrm{~min}$. Adapted with permission from ref. 89. Copyright 2017 Elsevier Ltd.

were facilely discriminated with this NIR probe, demonstrating the great potential of the probe for drug screening and cancer diagnosis.

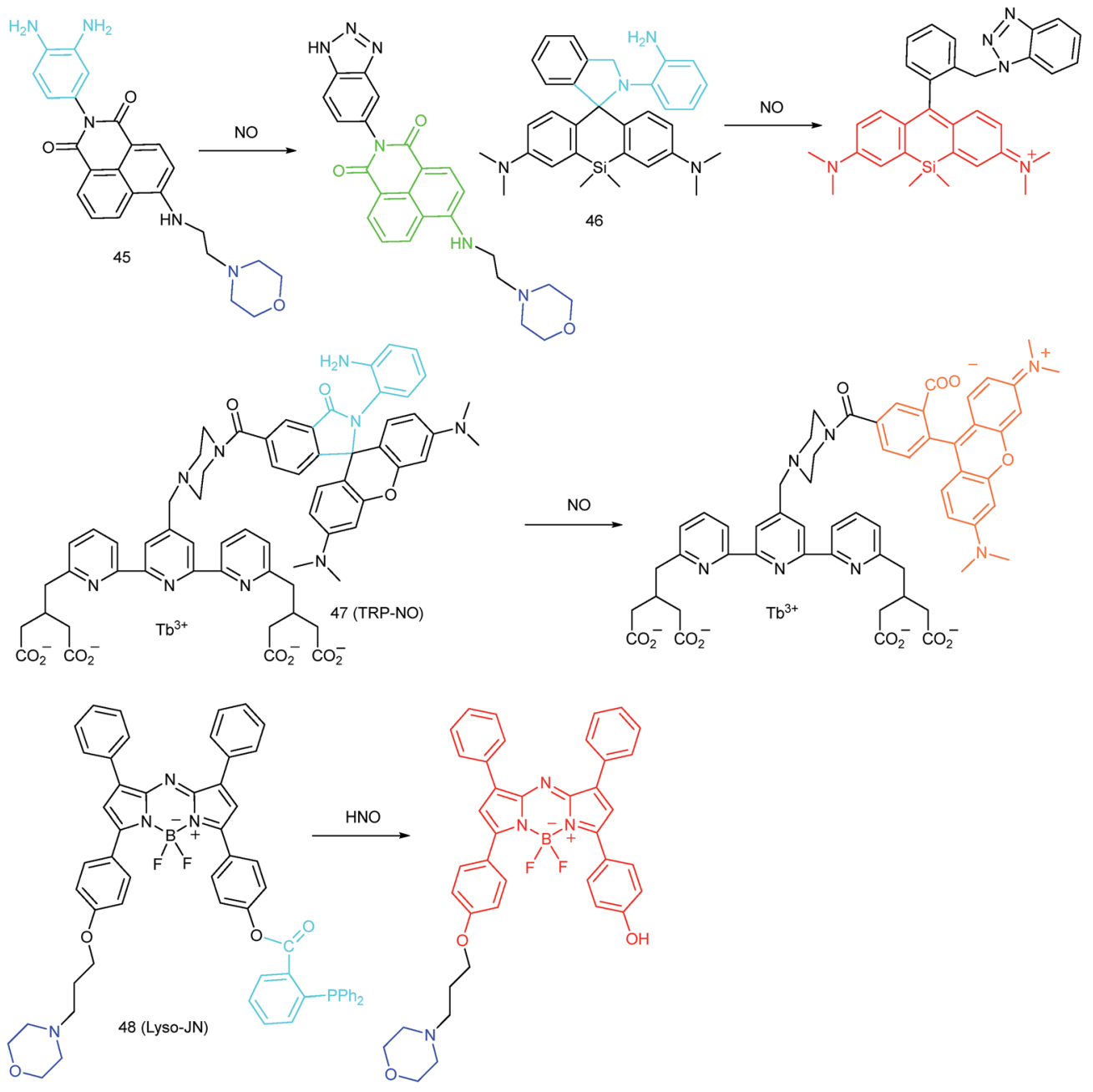

Fig. 21 Fluorescent probes for imaging RNS in lysosomes. 


\subsection{Imaging of RNS in lysosomes}

RNS in lysosomes are important for lysosomal microenvironment regulation. The overproduction of RNS in lysosomes can cause lysosomal nitrative stress and diverse disorders. ${ }^{\mathbf{4 8 b}, \boldsymbol{d}, \mathbf{5 0 a}, \mathbf{9 3}}$ Therefore, lysosomal RNS can be promising biomarkers for diverse diseases. For example, overproduction of NO in the lysosomes was discovered in diverse cancerous and inflamed cells. $^{93 b}$ Thus, lysosome targetable RNS probes are helpful for further understanding the biological and pathological functions of these molecules.

5.2.1. Imaging of NO in lysosomes. Our group reported a three-component lysosome targeted NO probe (45), which was prepared by tethering the NO trapping agent $o$-phenylenediamine to the imide position of the TP fluorophore naphthalimide, and the lysosome targeting moiety morpholine was introduced to the 4-position. ${ }^{93 b}$ The TP fluorescence of the probe was quenched due to the PET process between naphthalimide and $o$-phenylenediamine, but it recovered as a consequence of the generation of triazole from the NO and $o$ phenylenediamine trapper. 45 was used to capture the endogenous lysosomal NO in living cells for the first time, and the lysosomal NO generation under different stimulations was successfully monitored.

In 2017, Guo's group developed a Si-rhodamine deoxylactam-based fluorescent probe (46) for the detection of lysosomal NO. ${ }^{48 b}$ Benefiting from the characteristics of the PET mechanism and the Si-rhodamine fluorophore, this probe had a large fluorescence "off-on" ratio (6300-fold), strong emission intensity, low detection limit $(0.12 \mathrm{nM})$, fast response rate (within seconds) and high specificity. Interestingly, 46 localized in the lysosomes specifically owing to its easy protonation in mild acidic environments. Endogenous NO in a series of normal and inflamed cells as well as streptozotocin-treated mouse models was successfully visualized with this probe (Fig. 23).

Song and Yuan et al. developed a time-gated luminescence probe, 47 (TRP-NO), for in vivo NO imaging. ${ }^{48 d}$ The luminescence resonance energy transfer process from the luminescent

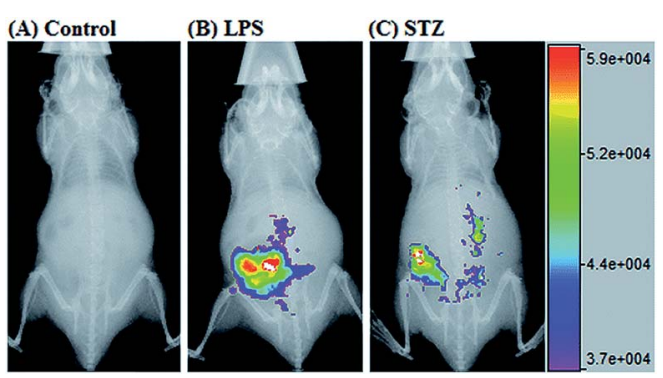

Fig. 23 Overlay of the fluorescence and X-ray images of mice. (A) The mouse was intraperitoneally injected with $46(100 \mu \mathrm{L}, 2 \mu \mathrm{M})$ for $30 \mathrm{~min}$. (B) The mouse was first intraperitoneally injected with LPS $\left(1 \mathrm{mg} \mathrm{mL}^{-1}\right.$, $100 \mu \mathrm{L})$ for $24 \mathrm{~h}$, and then intraperitoneally injected with $46(2 \mu \mathrm{M}, 100$ $\mu \mathrm{L}$ ) for $30 \mathrm{~min}$. (C) The mouse was first intraperitoneally injected with streptozotocin $\left(40 \mathrm{mg} \mathrm{kg}^{-1}\right)$ for $24 \mathrm{~h}$, and then intraperitoneally injected with $46(100 \mu \mathrm{L}, 2 \mu \mathrm{M})$ for $30 \mathrm{~min}$. Adapted with permission from ref. 48b. Copyright 2017 Royal Society of Chemistry.
$\mathrm{Tb}^{3+}$ complex to rhodamine was activated when the nonemissive spirolactam derivative was opened upon reacting with NO. 47 could be used for ratiometric and luminescence lifetime imaging, and the LOD of this probe was determined to be $1.8 \mu \mathrm{M}$. Interestingly, dye co-localization studies revealed that 47 had high affinity toward lysosomes. NO imaging in HepG2 cells and Daphnia magna was realized with this probe (Fig. 24).

5.2.2. Imaging of $\mathbf{H N O}$ in lysosomes. Chen et al. reported a BODIPY based NIR probe, 48 (Lyso-JN), for imaging of HNO in lysosomes. ${ }^{50 a}$ Morpholine was employed as the targeting group to endow the probe with lysosome-localization capacity; NIR emissive tetraarylazadipyrromethane was employed as the fluorophore because of its unique physiochemical properties; the diphenylphosphinobenzoyl group was chosen as the recognition moiety, which can quench the fluorescence of BODIPY, and can be specifically cleaved with HNO. 48 had high selectivity toward HNO among other bioactive species. Apparent fluorescent enhancement is observed after the cleavage of the diphenylphosphinobenzoyl group with HNO, and the LOD of 48 was calculated to be $0.06 \mu \mathrm{M} .48$ has been successfully employed for background-free fluorescence imaging of HNO in macrophage cells and living mice.

\subsection{Imaging of RSS in lysosomes}

RSS are also important for lysosomal microenvironment adjustment and cellular signal conduction. ${ }^{53 a, 94}$ Recently, some attention has been paid to the design and application of novel probes for lysosome-targeted detection of RSS. However, this type of probe is much rarer than mitochondrion-targeted ones (Fig. 25).

5.3.1. Imaging of thiols in lysosomes. In 2016, Peng et al. reported a naphthalimide based TP probe (49) for imaging thiols in lysosomes. ${ }^{94 b}$ This probe employed 2,4dinitrobenzene-sulfonyl (DNBS) as the responsive group and morpholine as the lysosome-targeting group. Before coming into contact with thiols, the fluorescence of 49 remained weak due to the PET process between DNBS and naphthalimide,

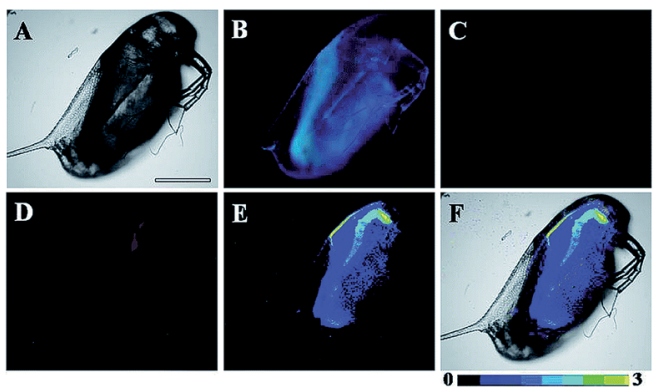

Fig. 24 Bright-field (A), steady-state (B) and time-gated ((C) $\mathrm{Tb}^{3+}$ luminescence; (D) rhodamine luminescence) luminescence images of

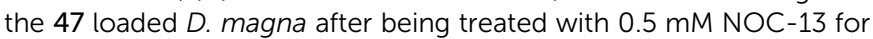
$10 \mathrm{~min}$. $(\mathrm{E})$ is the ratiometric (ratio $=I_{\text {red }} / I_{\text {green }}$ ) luminescence image of the $D$. magna. (F) is the merged image of brightfield and ratiometric images. Scale bar: $200 \mu \mathrm{m}$. Adapted with permission from ref. 48d. Copyright 2017 Royal Society of Chemistry. 


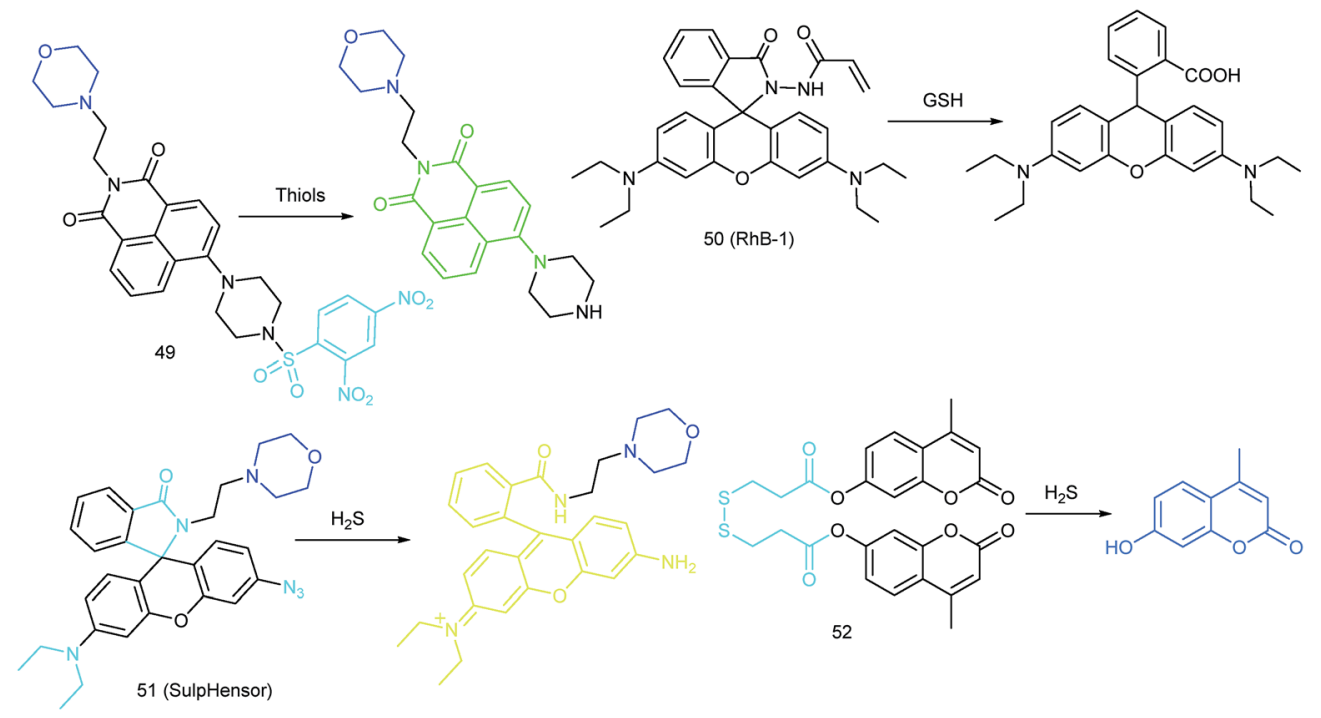

Fig. 25 Fluorescent probes for imaging RSS in lysosomes.

while significant fluorescence intensity enhancement was observed in the presence of thiols. The detection limits of $\mathbf{4 9}$ toward Cys, GS and Hcy are $2.6 \times 10^{-7} \mathrm{M}, 2.41 \times 10^{-6} \mathrm{M}$ and $4.87 \times 10^{-6} \mathrm{M}$, respectively. Colocalization experiments revealed that 49 localized in the lysosomes of cells specifically. Thiol imaging in living cells and tissues was successfully visualized using TP microscopy.

5.3.2. Imaging of GSH in lysosomes. Liu and Jiang et al. reported Rhodamine B-based lysosome-targeted probe $\mathbf{5 0}$ (RhB1) for GSH imaging. ${ }^{53 a}$ Based on the PET mechanism, the fluorescence of $\mathbf{5 0}$ was quenched before coming into contact with the analyte of interest. Benefiting from the high reactivity of acryloyl, the spirolactam ring of $\mathbf{5 0}$ was opened after the Michael addition reaction with GSH. The response time of this probe was less than $10 \mathrm{~s}$, and 16-fold fluorescence intensity enhancement is detected during the reaction between $\mathbf{5 0}$ and GSH. The LOD of $\mathbf{5 0}$ was $190 \mathrm{nM}$. Further experiments revealed that this probe is biocompatible and cell permeable, which indicated that $\mathbf{5 0}$ was a highly attractive "off-on" GSH probe for cell imaging. The lysosomal GSH in normal and NEM (sulfhydryl group blocker) treated HeLa cells was successfully distinguished with this probe.

5.3.3. Imaging of $\mathbf{H}_{2} \mathbf{S}$ in lysosomes. Yang et al. reported Rhodamine B-based probe $\mathbf{5 1}$ (SulpHensor). ${ }^{94 a}$ Morpholine was introduced as the targeting group, and the spirolactam moiety and azide group were employed to quench the fluorescence of the probe. The spirolactam moiety of $\mathbf{5 1}$ was opened in lysosomal acidic microenvironments, while the azide group was specifically reduced by $\mathrm{H}_{2} \mathrm{~S}$. Therefore, the fluorescence of the probe was only turned on in acidic environments in the presence of $\mathrm{H}_{2} \mathrm{~S}$, which was beneficial for enhancing the lysosome specificity and avoiding the undesired background fluorescence during cell imaging. Lysosomal $\mathrm{H}_{2} \mathrm{~S}$ imaging in HeLa cells with different stimulations was realized with this probe.
Zhou and Zhang et al. reported a coumarin based probe (52) for lysosome-targeted $\mathrm{H}_{2} \mathrm{~S}$ imaging. ${ }^{95} \mathbf{5 2}$ was facilely prepared by connecting two coumarin fluorophores with disulfide bonds. Before the reaction with $\mathrm{H}_{2} \mathrm{~S}$, the emission of $\mathbf{5 2}$ remained weak. However, after coming into contact with $\mathrm{H}_{2} \mathrm{~S}, 15$-fold fluorescence enhancement was observed due to the specific cleavage of disulfide bonds. Interestingly, co-localization experiments demonstrated the lysosome-targeting capability of 52. Fluorescence imaging of endogenous and exogenous $\mathrm{H}_{2} \mathrm{~S}$ in HeLa cells, D. melanogaster and C. elegans was successfully realized with this probe.

\subsection{Challenges for lysosome-targeted probes}

Significant progress has been made in the development of lysosome-targeted fluorescent probes in recent years. However, there are still several limitations for related probes. For example, the targeting mechanisms of most developed probes are the trapping of protonated lipophilic amines, which cannot be used to distinguish between endosomes, autophagosomes, autolysosomes and other acidic compartments. Moreover, these ligands are toxic to living cells because they can alkalize lysosome microenvironments and hinder the smooth progress of diverse lysosome activities, which make them unsuitable for long-term tracing. Therefore, lysosome selective probes without the alkalization effect are required. As discussed above, genetically encoded probes need to be frequently washed to diminish the background during imaging, and this can also influence the lysosomal microenvironments. Therefore, designing bioorthogonal probes with strong anti-interference capability, excellent selectivity and high sensitivity is still hard. Moreover, some probes may be quenched in the acidic lysosomal microenvironments, which poses extra challenges for designing related probes. Most of the current developed probes emit in the visible region, indicating that they cannot be used for deep tissue imaging due to their poor penetration capacity. 


\section{Endoplasmic reticulum targeted probes}

The ER is the main place for protein fabrication, modification, folding and transportation. It also takes charge of the synthesis of other molecules, like lipids and carbohydrates, as well as $\mathrm{Ca}^{2+}$ storage. ${ }^{30 b, 96}$ During synthesis of diverse proteins, RONSS are indispensable for controlling their functional threedimensional structures and chemical groups. ${ }^{50 b, 97}$ Therefore, maintaining suitable oxidative microenvironments is essential for ER functions. Once the redox homeostasis in the ER is out of control, it can hamper the formation of proper protein structures and cause diverse disorders as well as ER stress. ${ }^{\mathbf{3 0 a}, \mathbf{9 6 b}, \boldsymbol{c}}$ Development of ER-targeted probes and tracing localized RONSS has been recognized as a new strategy for investigating the molecular mechanisms of different diseases.

\subsection{Imaging of ROS in the endoplasmic reticulum}

ROS are critical for protein synthesis because they are involved in the formation of the disulfide bond, which is essential for protein folding. However, overexpression of ROS in the ER may cause oxidative stress, which can lead to protein crosslinking and further result in morbigenous effects on living cells. Developing novel fluorescent probes capable of tracing the ROS flux in the ER is significant for revealing the potential physiological and pathological functions of ROS during different bioevents, which should also help to improve the understanding of different major diseases (Fig. 26).

6.1.1. Imaging of $\mathrm{O}_{2}{ }^{--}$in the endoplasmic reticulum. Recently, Xiao et al. reported an ER-targeted TP probe, $\mathrm{O}_{2}{ }^{-}{ }^{-} \mathbf{5 3}$ (ER-BZT). ${ }^{30 a}$ In this probe, $p$-toluene sulfonamide was employed as the targeting group, naphthalimide as the fluorophore and benzothiazoline as the responsive group. The fluorescence of naphthalimide was quenched based on the PET mechanism.

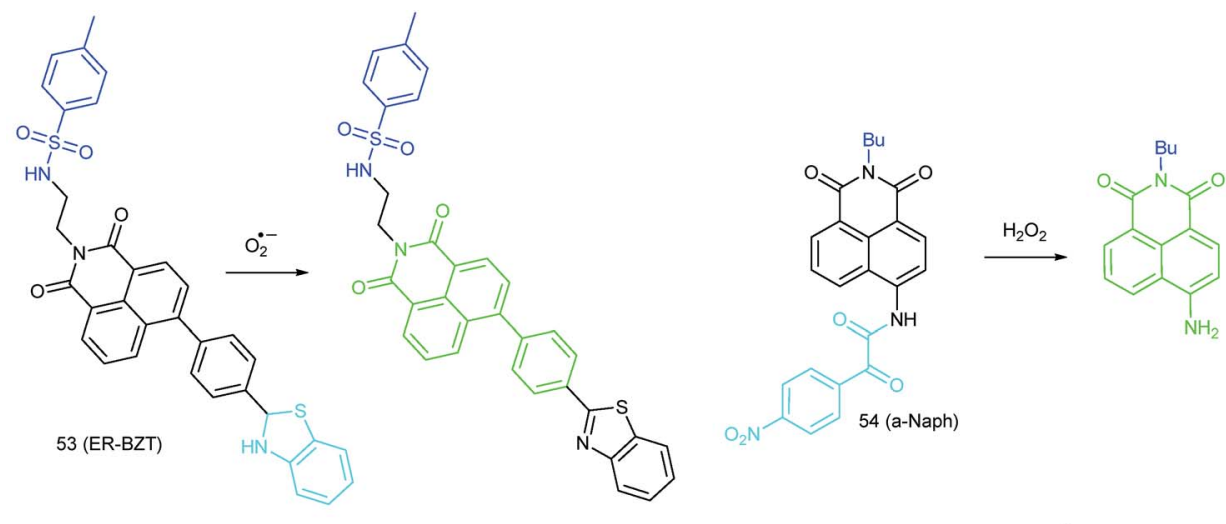<smiles></smiles>

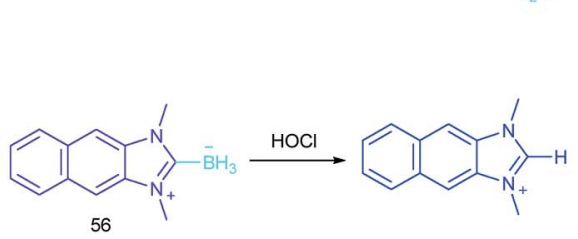
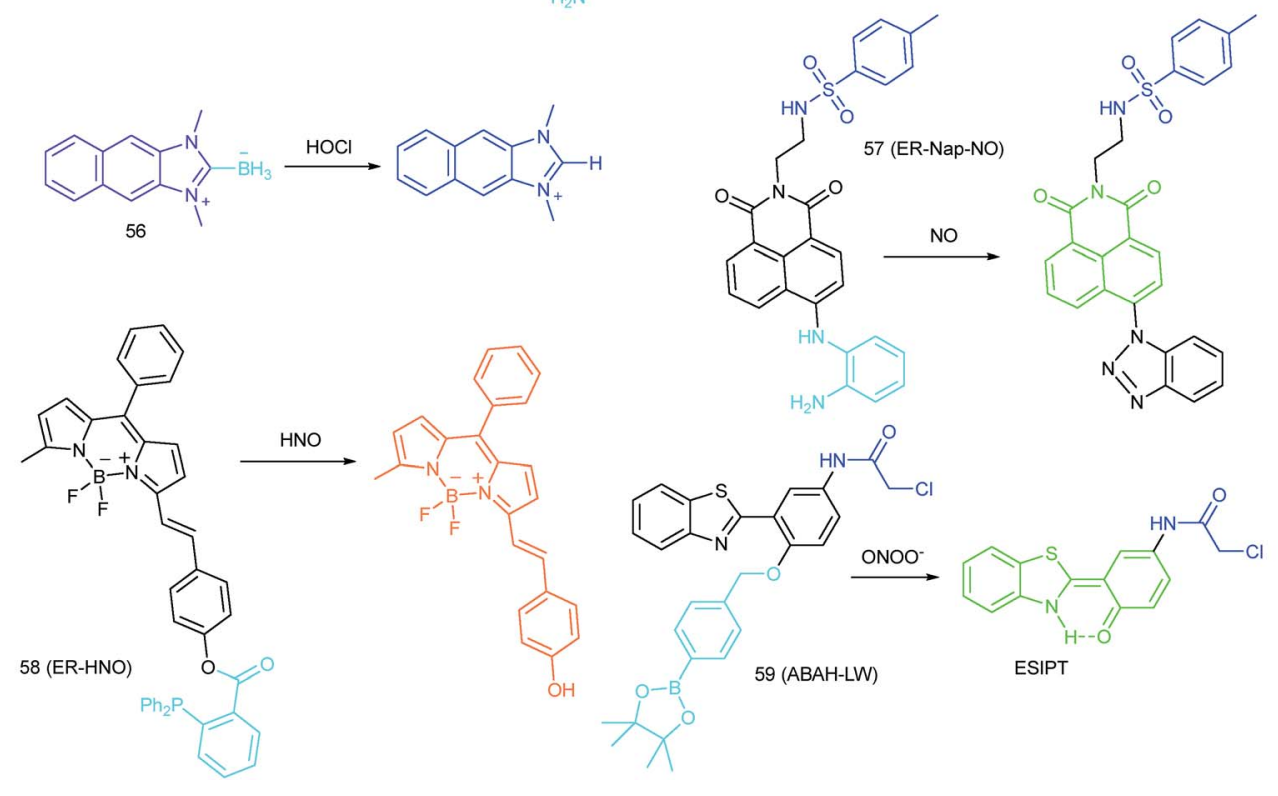

Fig. 26 Fluorescent probes for imaging of ROS and RNS in the ER. 
Upon the addition of $\mathrm{O}_{2}{ }^{--}$, dramatic fluorescence enhancement was observed from the probe solution, as a consequence of the dehydrogenation reaction between $\mathrm{O}_{2}{ }^{-}$and benzothiazoline disrupting the PET process. The probe localized in the ER well (Fig. 27) and the LOD of 53 was determined to be $60 \mathrm{nM} . \mathrm{O}_{2}{ }^{--}$ burst in the ER of HepG2 cells during ER stress was detected with this probe, and the higher endogenous $\mathrm{O}_{2}{ }^{-}$level in abdominal and hepatic tissue of diabetic mice was demonstrated with 53 by using TP microscopy, which indicated that this probe holds promise for revealing the chemical-biological roles of $\mathrm{O}_{2}{ }^{\cdot-}$ in diverse ER-associated diseases.

6.1.2. Imaging of $\mathrm{H}_{2} \mathrm{O}_{2}$ in the endoplasmic reticulum. In 2017, Zhang and co-workers reported another naphthalimide based probe, $\mathbf{5 4}$ ( $\alpha$-Naph), for selectively tracing the $\mathrm{H}_{2} \mathrm{O}_{2}$ in the ER. ${ }^{97 a}$ In this probe, $\alpha$-ketoamide was selected as the $\mathrm{H}_{2} \mathrm{O}_{2}$ responsive group. Upon addition of $\mathrm{H}_{2} \mathrm{O}_{2}$ to the solution of 54, the $\alpha$-ketoamide was converted into a stronger electron donating amino group, resulting in a ratiometric fluorescence signal change. The LOD of $\mathbf{5 4}$ is $38 \mathrm{nM}$ and confocal imaging experiments revealed that $\mathbf{5 4}$ localized in the ER well. With this probe, the basal $\mathrm{H}_{2} \mathrm{O}_{2}$ concentration of HeLa cells in the ER was detected (estimated to be $0.692 \mu \mathrm{M}$ ), and it was found to be elevated to $1.26 \mu \mathrm{M}$ under PMA stimulation.

6.1.3. Imaging of $\mathrm{HOCl}$ in the endoplasmic reticulum. Using $p$-toluene sulfonamide as the targeting group, Kim and co-workers reported a curcumin based ratiometric probe, $\mathbf{5 5}$ (ER-ClO) ${ }^{96 c} 55$ had a maximum absorption peak at $488 \mathrm{~nm}$ and emitted at $554 \mathrm{~nm}$. After reacting with $\mathrm{HOCl}$, the cleavage of diaminomaleonitrile resulted in a shift of the main adsorption peak to $437 \mathrm{~nm}$, while the emission peak blue-shifted to $480 \mathrm{~nm}$. This probe had excellent selectivity toward $\mathrm{HOCl}$, with a LOD of $0.59 \mu \mathrm{M}$. 55 has been successfully employed for ratiometric tracing of endogenous $\mathrm{HOCl}$ in the ER of HeLa cells under the PMA stimuli.

ER-selective fluorescent probes based on other groups have also been developed. Yoon, Bouffard and Kim reported

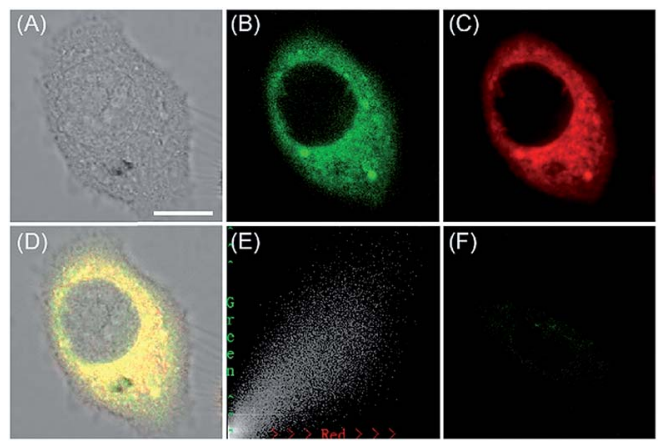

Fig. 27 The colocalization imaging of 53 and ER-tracker Red in Tmtreated HepG2 cells. (A) The bright-field image. (B) The fluorescence image of $53(10 \mu \mathrm{M}, \mathrm{Ex}=405 \mathrm{~nm}$, collected $420-480 \mathrm{~nm})$. (C) The fluorescence image of ER-Tracker Red $(0.5 \mu \mathrm{M}$, Ex $=543 \mathrm{~nm}$, collected 580-630 nm). (D) The merged image of (A)-(C). (E) Intensity correlation plot of stain 53 and ER-Tracker Red. (F) The fluorescence image of ER-BZT in Tm-induced HepG2 cells pretreated with Tiron $(5.0 \mathrm{mM})$ for $20 \mathrm{~min}$. Scale bar: $10 \mu \mathrm{m}$. Adapted with permission from ref. 30a. Copyright 2016 Royal Society of Chemistry.
$\mathrm{N}$-heterocyclic carbene borane-based HOCl probe 56 with ERtargeting capability. ${ }^{98}$ In the presence of $\mathrm{HOCl}$, the specific oxidation of the B-H bond occurred, yielding the emissive naphthoimidazolium derivative and a 470-fold ratiometric signal response. Confocal imaging experiments revealed that probe 56 overlapped well with commercialized ER-Tracker Red (Fig. 28), and HOCl imaging in RAW264.7 cells as well as rat hippocampal slices was realized.

\subsection{Imaging of RNS in the endoplasmic reticulum}

Although fluorescent probes for tracing RNS in living cells have attracted significant interest during the past few decades, probes capable of imaging the RNS fluctuations in subcellular structures are still rare, especially in the ER. However, ER nitrative stress caused by RNS overproduction also has deadly effects on living cells. Therefore, ER-targeted RNS probes are urgently needed due to their potential in early warning and diagnosis. Several groups have devoted efforts and made progress in related areas in the past 3 years.

6.2.1. Imaging of NO in the endoplasmic reticulum. Very recently, $\mathrm{Li}$ and co-workers devised an ER-targeted NO probe, $\mathbf{5 7}$ (ER-Nap-NO), based on the $p$-toluene sulfonamide and $o$-phenylenediamino (OPD) functionalized naphthalimide platform. ${ }^{\mathbf{9} \boldsymbol{a}}$ Initially, the fluorescence of naphthalimide was quenched by the OPD moiety. In the presence of $\mathrm{NO}$ and $\mathrm{O}_{2}$, significant turn-on fluorescence could be observed due to the prohibition of PET. 57 was applied for TP imaging of the NO level in the ER of HeLa cells, mouse liver tissues and leg tissues through TP microscopy (Fig. 29), which offered an alternative candidate for investigating the relationships between NO and ER associated diseases.

6.2.2. Imaging of $\mathrm{HNO}$ in the endoplasmic reticulum. Das's group synthesized a BODIPY based fluorescent probe, $\mathbf{5 8}$ (ER-HNO), for imaging HNO in the ER. ${ }^{50 b}$ The fluorescence of 58 was faint due to the quenching capability of the diphenylphosphinobenzoyl group, and was recovered upon the specific
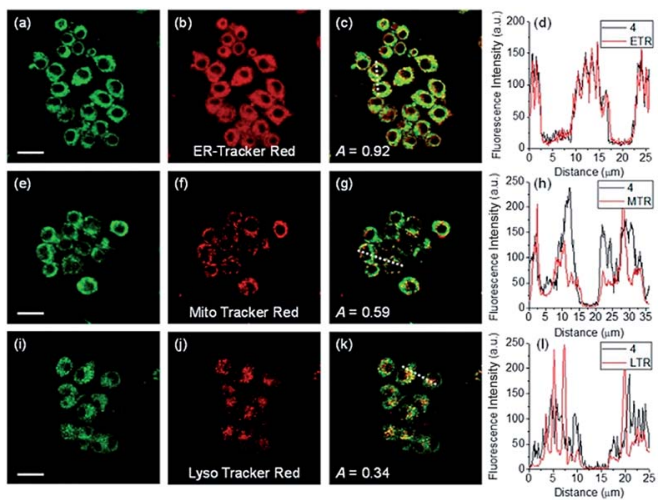

Fig. $28 \operatorname{TPM}(a, e$, and $\mathrm{i})$, OPM (b, f, and j), and merged (c, g, and $k$ ) images of RAW264.7 cells colabeled with $56(10 \mu \mathrm{M})$ and organelle trackers $(1.0 \mu \mathrm{M})$. Line profile of fluorescence intensity ( $d, h$, and l) obtained from the corresponding cells images. Scale bars $=20 \mu \mathrm{m}$. Adapted with permission from ref. 98. Copyright 2016 Royal Society of Chemistry. 

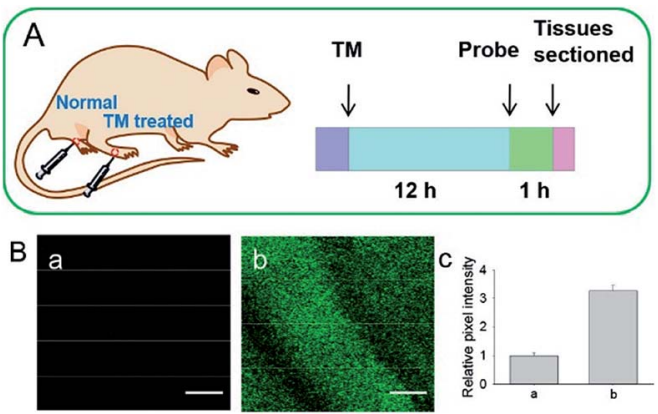

Fig. 29 (A) Preparation of normal tissues and tunicamycin-treated tissues from a mouse model. (B) Two-photon fluorescence images of tissues during ER stress: (a) normal tissues, (b) tunicamycin-treated tissues, and (c) relative pixel intensities for images (a) and (b). Scale bar is $100 \mu \mathrm{m}$. Adapted with permission from ref. 96a. Copyright 2016 Royal Society of Chemistry.

Staudinger reaction with HNO. Benefiting from the photostability of BODIPY, this probe was suitable for structured illumination microscopy. Colocalization experiments revealed that the probe localized in the ER of macrophage cells specifically. 52 was employed in the visualization of HNO in living cells as well as Artemia nauplii.

6.2.3. Imaging of $\mathrm{ONOO}^{-}$in the endoplasmic reticulum. $\mathrm{ONOO}^{-}$is known for its toxic effect on the ER, which can deplete the $\mathrm{Ca}^{2+}$ in the ER while elevating the cytosol $\mathrm{Ca}^{2+}$ and inducing cell apoptosis. ${ }^{\mathbf{9 6 b}} \mathrm{ONOO}^{-}$overproduction in the ER is closely related to numerous disorders including atherogenesis. Recently, James et al. reported an ESIPT based ERtargeted fluorescent probe, 59 (ABAH-LW), for $\mathrm{ONOO}^{-}$ imaging. ${ }^{96 \boldsymbol{b}}$ The ESIPT process in $\mathbf{5 9}$ was first blocked by the benzyl boronic ester group, which resulted in the probe only emitting the enol emission peak at $405 \mathrm{~nm}$. Addition of $\mathrm{ONOO}^{-}$specifically released the keto fluorophore, accompanied by a 103-fold ratiometric fluorescence enhancement $\left(F_{481} / F_{405}\right)$. The LOD of 59 was calculated to be $21.4 \mathrm{nM}$ and this probe had been successfully employed for tracing the $\mathrm{ONOO}^{-}$in the ER of HeLa cells under LPS and IFN- $\gamma$ stimulation. As the first ER-targeted $\mathrm{ONOO}^{-}$probe, 59 may offer new ideas for investigation of the chemical-biological functions of $\mathrm{ONOO}^{-}$.

\subsection{Imaging of RSS in the endoplasmic reticulum}

ER-targeted RSS probes are beneficial for mapping the fluctuation of RSS during diverse processes, and this can offer direct evidence for the cellular functions of RSS. Related investigations have gradually received attention, and several probes have been devised and successfully used for RSS imaging in the ER. But it should be recognized that this is still in its infancy, there is plenty of room for better performance fluorescent probes.

6.3.1. Imaging of thiols in the endoplasmic reticulum. Recent studies have revealed that depression phenotypes might be associated with oxidative stress-related ER disorders. In view of the antioxidant function of thiols, tracing thiols in the ER should have implications for depression investigations.
Recently, our group developed a PET based TP probe, 60 (ER$\mathrm{SH}$ ), with ER-targeting capability, for selective thiol imaging. ${ }^{30 c}$ With the assistance of TP microscopy, this probe successfully revealed the reduced thiol levels in the ER of mice brains with depression phenotypes.

6.3.2. Imaging of $\mathrm{H}_{2} \mathrm{~S}$ in the endoplasmic reticulum. Lin's group reported the first ER-targeted $\mathrm{H}_{2} \mathrm{~S}$ probe, $61\left(\mathrm{Na}-\mathrm{H}_{2} \mathrm{~S}\right.$ ER), ${ }^{99}$ which could selectively image $\mathrm{H}_{2} \mathrm{~S}$ in the ER of HeLa cells based on the ICT mechanism: the fluorescence of the probe was firstly quenched by the azide group and the probe could localize into the ER under the direction of $p$-toluene sulfonamide; after coming into contact with $\mathrm{H}_{2} \mathrm{~S}$, the azide can be reduced into an amino group, resulting in the formation of a " $D-\pi-A$ " structure and producing "turn-on" fluorescence at $545 \mathrm{~nm}$. 61 was demonstrated to be capable of tracing $\mathrm{H}_{2} \mathrm{~S}$ in the ER of living cells and tissues as well as zebrafish.

6.3.3. Imaging of $\mathrm{H}_{2} \mathrm{~S}_{n}$ in the endoplasmic reticulum. By virtue of the reactivity of $\mathrm{H}_{2} \mathrm{~S}_{n}$, more and more investigations find that $\mathrm{H}_{2} \mathrm{~S}_{n}$ may be the actual signal molecule, and is associated with numerous physiological and pathological phenomena. However, the implication of $\mathrm{H}_{2} \mathrm{~S}_{n}$ in the ER function has rarely been checked due to the lack of ideal probes.

Recently, Das, Thomas and co-workers devised probe 62 (MB-S ${ }_{n}$ ) for ER $\mathrm{H}_{2} \mathrm{~S}_{n}$ imaging. ${ }^{97 c}$ The fluorescence of the probe is blocked initially. Upon reacting with $\mathrm{H}_{2} \mathrm{~S}_{n}$, the cleavage of a thioester and subsequent nucleophilic attack occurs, releasing a fluorophore. The LOD of 62 was as low as $26.01 \mathrm{nM}$. Benefiting from the excellent photostability and ER-targeting capability, 62 was employed for imaging $\mathrm{H}_{2} \mathrm{~S}_{n}$ in the ER of living cells with the assistance of super-resolution structured illumination microscopy, which showed promise for mapping the intracellular distribution of $\mathrm{H}_{2} \mathrm{~S}_{n}$ and further exploring their biological functions. Very recently, Zhou's group reported a probe, $63\left(\right.$ ER- $\left.\mathrm{H}_{2} \mathrm{~S}_{2}\right)$, and successfully traced $\mathrm{H}_{2} \mathrm{~S}_{2}$ in the ER of living cells as well as zebrafish. ${ }^{\mathbf{1 0 0}}$

\subsection{Challenges for endoplasmic reticulum targeted probes}

Over the past three years, a series of ER-targeted fluorescent probes have been developed for selectively staining bioactive species in different research models, such as diabetes, inflammation and depression. However, the targeting mechanisms of ER-selective probes are still unknown, which severely limited our pursuit of better performing fluorescent tools and further understanding the chemical-biological functions of different species. Conjugating responsive groups with ER-targeted dyes seems to be feasible, but the subtle alteration of molecular charges and bulk usually hampers the localization capability. The protein tagging technique has also been employed to resolve the localization of fluorescent probes, but the complexity as well as efficiency remain to be further resolved. Moreover, almost all the reported probes emit visible light, and the in vivo applications of these probes are limited. Many bioactive species still cannot be visualized in the ER due to the lack of applicable probes. Probes for the quantification of the basal concentrations of diverse species in the ER are also rare (Fig. 30). 

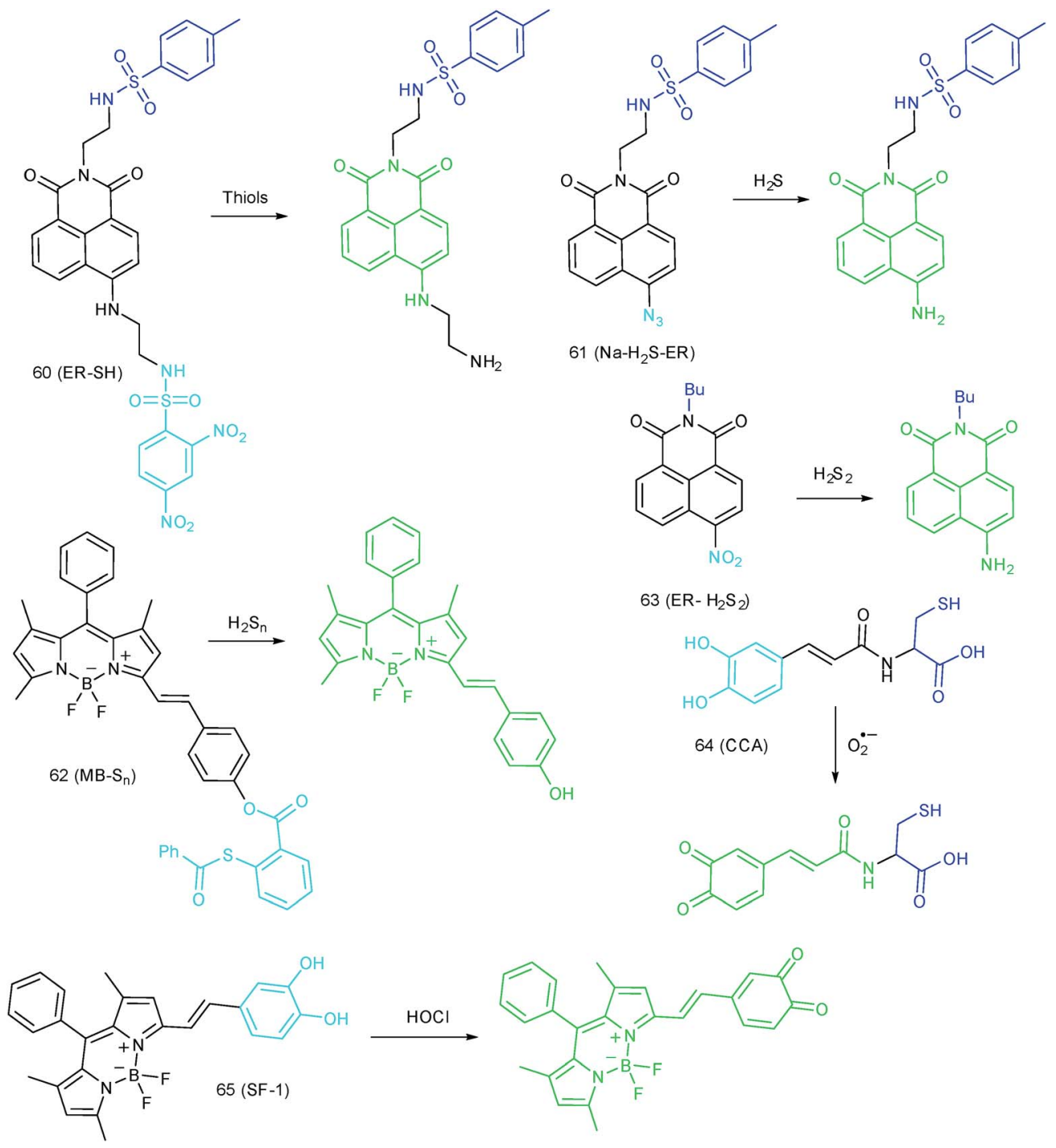

Fig. 30 Fluorescent probes for imaging RSS in the ER (60-63) and ROS in the Golgi apparatus (64 and 65).

\section{Golgi apparatus-targeted probes}

To reveal the intrinsic molecular mechanisms of hepatic IRI, our group developed an L-cysteine containing TP probe, 64 (CCA), for reversible imaging of the $\mathrm{O}_{2}{ }^{--}$fluctuation in the Golgi apparatus. ${ }^{31 a} 64$ was prepared by connecting caffeic acid with $\mathrm{L}^{-}$ cysteine through an amide bond. When reacting with $\mathrm{O}_{2}{ }^{--}$, the fluorescence intensity of $\mathbf{6 4}$ increased linearly to the change of $\mathrm{O}_{2}{ }^{\cdot-}$ concentration in the range of $0-5 \mu \mathrm{M}$, and the LOD of this probe was estimated to be $18 \mathrm{nM}$. Benefiting from the abundance of cysteine residue recognition receptors in the Golgi apparatus, probe 64 could target this organelle effectively. With this probe, the significant increase of $\mathrm{O}_{2}{ }^{-}$levels in the Golgi apparatus of mice liver during IRI was disclosed by using TP microscopy. More significantly, the tumor necrosis factor (TNF$\alpha$ ) as the positive $\mathrm{O}_{2}{ }^{-}$mediator was demonstrated (Fig. 31). Therefore, 64 not only revealed the molecular mechanism of hepatic IRI, but also provided a promising treatment strategy, which further proved the significance of organelle-targeted fluorescent probes for disease diagnosis and drug discovery.

Das, Thomas and Smythe et al. reported BODIPY derivative probe 65 (SF-1) for $\mathrm{HOCl}$ imaging. ${ }^{97 b}$ Interestingly, the low charge/intermediate amphiphilicity made 65 selectively accumulate in the Golgi apparatus and lysosomes, and "turn-on" fluorescence is observed only upon addition of $\mathrm{HOCl}$ into the probe solution, with a LOD of $4.3 \mathrm{nM}$. Endogenous $\mathrm{HOCl}$ in the Golgi apparatus and lysosomes of macrophage cells under LPS stimulation was visualized with 65 with the assistance of structured illumination microscopy, with a lateral resolution of $120 \mathrm{~nm}$.

\subsection{Challenges for Golgi apparatus-targeted probes}

Golgi apparatus-targeted RONSS fluorescent probes have not received enough interest in the past decade, mostly due to the lack of efficient targeting ligands. Although several probes have been reported for imaging metal ions in the Golgi apparatus, 

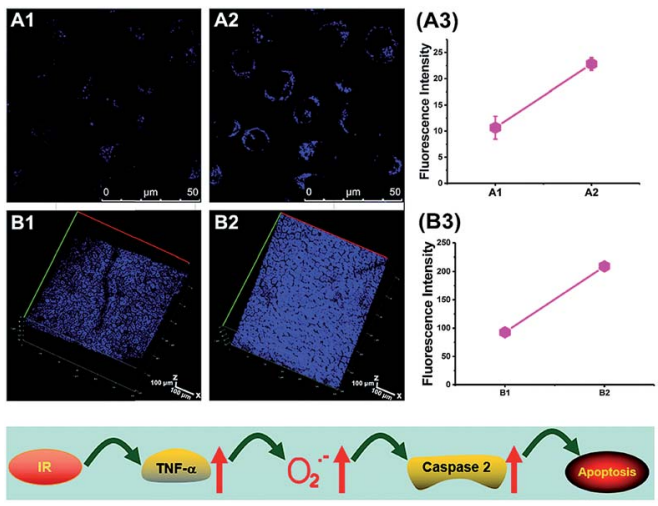

caspase 2

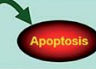

Fig. 31 Top: Increased $\mathrm{O}_{2}{ }^{\cdot-}$ levels in IR cells and mice. (A) The normal (A1) and IR hepatic (A2) cells loaded with $10 \mu \mathrm{M} 64$ for TP fluorescence imaging, and (A3) the average fluorescence intensity output of (A). (B) The in vivo 3D images of normal (B1) and IR mice (B2) injected with 10 $\mu \mathrm{M} 64$ for TP fluorescence imaging, and (B3) the average fluorescence intensity output of (B). Images were acquired at an excitation wavelength of $800 \mathrm{~nm}$ and emission wavelengths corresponding to the blue channel of 430-530 nm. Bottom: Model for the signalling role of $\mathrm{O}_{2}{ }^{--}$in IR cells and mice. Adapted with permission from ref. 31a. Copyright 2019 Royal Society of Chemistry.

the targeting mechanisms should be explored for further accelerating the development of related probes. L-Cysteine has been proved to be a promising targeting ligand, but the universality remains to be further checked. Though enzymeactivated probes hold promise for localization selective imaging, the scope has not been extended to Golgi apparatustargeted RONSS probes.

\section{Probes for multi-parameter imaging}

Apart from the above-discussed probes that can only image one species in different organelles, probes for sequential or simultaneous tracing of two or more targets have been recognized to be another group of important tools for bioanalysis, owing to their potential in clarifying the inter-conversion and "crosstalk" between different species as well as different organelles. ${ }^{8 c, 101}$ Such interesting tasks can be realized by using fluorescent probes with two or more responsive groups or employing several probes without spectral overlap. Nanoprobes containing several recognition molecules are also alternative candidates.

\subsection{Simultaneous imaging of bioactive species in an organelle}

RONSS are highly reactive, interactive and interconvertible molecules. Sequential or simultaneous imaging of two or more species can reveal their internal relationships in specific organelles and further advance our understanding of diverse biological events. ${ }^{30 b, 101 c, 102}$ Probes for sequential or simultaneous imaging can be applied for tracing diverse critical biological events. For example, probes capable of simultaneous imaging are helpful for monitoring the real-time distribution of different species, and they can even be explored to identify the "crosstalk" between different subcellular structures. On the other hand, probes for sequential imaging are helpful for revealing the upstream and downstream relationships between different species. Recently, several probes have been developed for organelle-targeted multiple analyte imaging, and they can be divided into molecular probes and nanoprobes (Fig. 32).

8.1.1. Molecular probes. It was reported that $\mathrm{O}_{2}{ }^{-}$can directly react with $\mathrm{H}_{2} \mathrm{~S}$ to generate $\mathrm{H}_{2} \mathrm{~S}_{n}$, and sulfane sulfur might be the real signalling molecule in living cells. Therefore, fluorescent probes that can sequentially image $\mathrm{O}_{2}{ }^{--}$and $\mathrm{H}_{2} \mathrm{~S}_{n}$ in subcellular structures are useful for tracing this important biosynthetic reaction. Chen et al. reported a NIR probe, 66 (HCyFN), with dual responsive sites for sequential $\mathrm{O}_{2}{ }^{-}$and $\mathrm{H}_{2} \mathrm{~S}_{n}$ imaging in mitochondria. ${ }^{\mathbf{1 0 2}}$ Heptamethine cyanine was employed as the fluorophore due to its NIR emissive features, and nitro-activated fluorobenzoiate was used as the $\mathrm{H}_{2} \mathrm{~S}_{n}$ recognition moiety because of its bis-electrophilic reaction characteristic toward $\mathrm{H}_{2} \mathrm{~S}_{n}$. The $\mathrm{O}_{2}{ }^{\cdot-}$ mediated hydrogen abstraction reaction could recover the conjugated system of the hydrocyanine, which caused the fluorescence intensity enhancement at $794 \mathrm{~nm}$; when the product came into contact with $\mathrm{H}_{2} \mathrm{~S}_{n}$, the specific biselectrophilic reaction revoked the d-PET process, and the released fluorophore had a maximum emission wavelength of $625 \mathrm{~nm}$. With 66, $\mathrm{O}_{2}{ }^{--}$directed $\mathrm{H}_{2} \mathrm{~S}$ conversion was demonstrated to be a promising $\mathrm{H}_{2} \mathrm{~S}_{n}$ biosynthetic pathway through the mitochondrion-targeted dual-channel imaging in exogenous superoxide and NaHS treated macrophages.

The inter-relationship of mitochondrial $\mathrm{O}_{2}{ }^{--}$and $\mathrm{pH}$ is unclear for cellular signal transduction and homeostasis. Novel probes for precise evaluation of the subcellular $\mathrm{pH}$ and ROS fluctuation in the mitochondria are beneficial for revealing their interactions. A TP probe, 67 (CFT), was reported by our group. ${ }^{102 d} 67$ is prepared by covalent linkage of caffeoyl, fluorescein and TPP. This probe could make distinguishable optical signal changes upon coming into contact with different analytes. Benefiting from the reversible response and specificity, instantaneous, dynamic and reversible monitoring of the concentration fluctuations of $\mathrm{H}^{+}$and $\mathrm{O}_{2}{ }^{-}$in mitochondria of 4T1 cells was achieved with the assistance of TP microscopy. Importantly, with this probe, Drp1 deficiency was revealed to be a promising signal pathway to induce mitochondrial hyperfusion and subsequent cell apoptosis.

Unlike the complicated design of multi-responsive probes, simultaneous employment of different probes with distinguishable spectral properties to detect bioactive species in specific organelles is more feasible and easier to perform. Using probe 68-1 $\left(\mathrm{M}-\mathrm{H}_{2} \mathrm{O}_{2}, \mathrm{H}_{2} \mathrm{O}_{2}\right.$ probe, LOD = $\left.18 \mathrm{nM}\right)$ and 68-2 $(\mathrm{M}-$ $\mathrm{Zn}, \mathrm{Zn}^{2+}$ probe, LOD $=11 \mathrm{nM}$ ), simultaneous imaging of $\mathrm{H}_{2} \mathrm{O}_{2}$ and $\mathrm{Zn}^{2+}$ fluctuation in the mitochondria of living cells was realized by our group. ${ }^{102 b}$ In vitro and in vivo tests revealed the synergistic concentration enhancement of $\mathrm{H}_{2} \mathrm{O}_{2}$ and $\mathrm{Zn}^{2+}$ in mitochondria during apoptosis and inflammation (Fig. 33), and this offered a new idea for investigating the correlations between different bioactive species in diverse organelles of living cells.

8.1.2. Nanoprobes. Nanomaterials are novel platforms for constructing organelle-targeted probes to trace multiple 

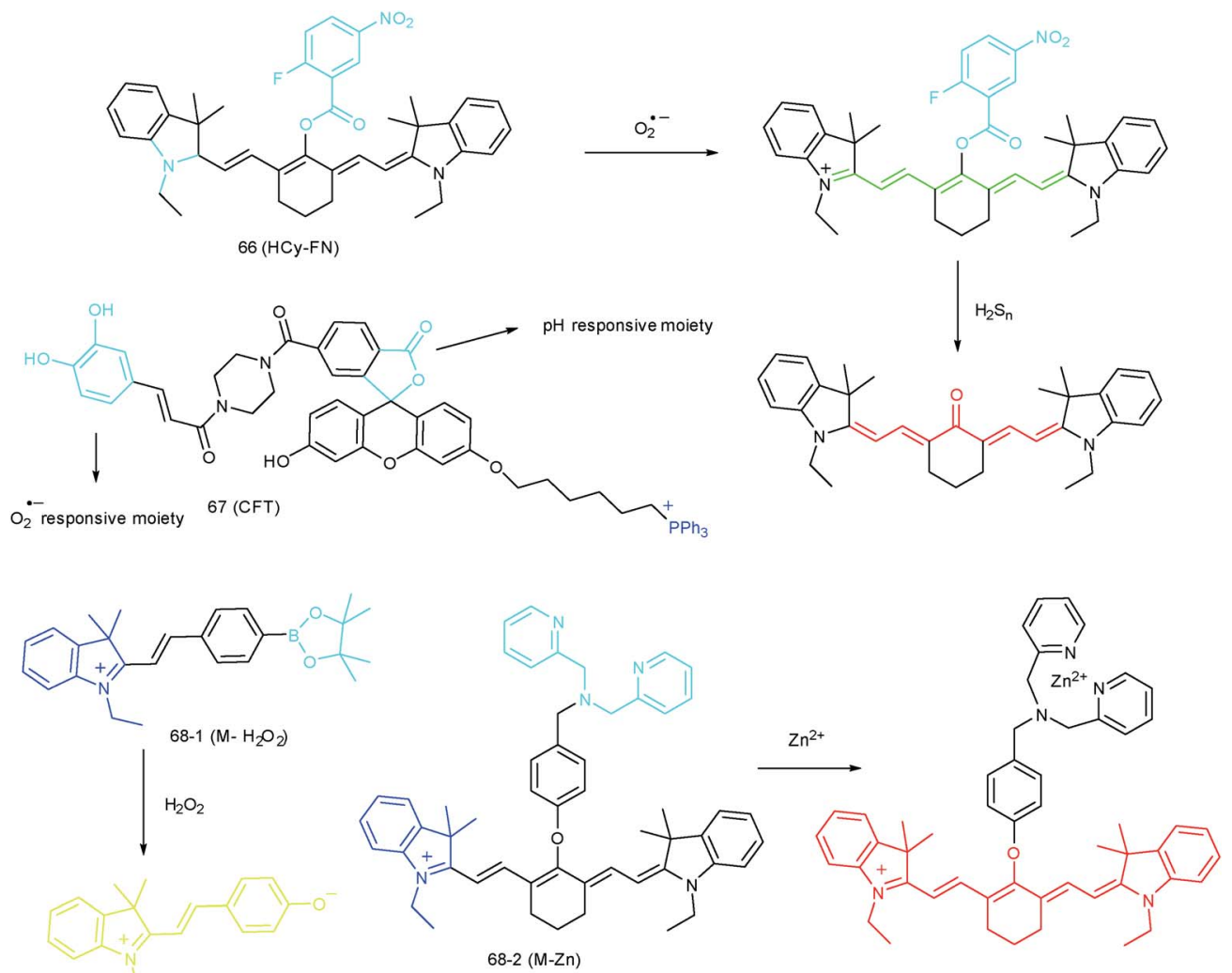

Fig. 32 Molecular probes for simultaneous imaging of bioactive species in one organelle.

analytes during different biological events, because they can simultaneously connect abundant reporter molecules and targeting groups. ${ }^{8 c, 101 a, b, 103}$ Our group developed a mitochondriontargeted silica based nanoprobe (69) for simultaneously imaging the fluctuation of $\mathrm{H}_{2} \mathrm{O}_{2}$ and $\mathrm{pH}$ in living cells. ${ }^{101 c} \mathrm{H}_{2} \mathrm{O}_{2}$ probe $\mathrm{Cy}-\mathrm{O}-\mathrm{SeH}$ and $\mathrm{pH}$ probe fluorescein were loaded into the MSNs. To further prevent the leakage of the dyes and endow the probes with mitochondrion-targeting capability, PEI and TPP were grafted on the surface of MSNs. The final obtained nanoprobe had an average size of $30 \mathrm{~nm}$. Mitochondrial $\mathrm{H}_{2} \mathrm{O}_{2}$ and $\mathrm{pH}$ fluctuation in MCF-7 and MCF-10A cells under different stimulations were successfully tracked with 69. This work showed the great promise of organelle-targeted nanoprobes for bioanalysis (Fig. 34).

A QD based ratiometric probe (70) was developed by Tian's group. ${ }^{104}$ Silica encapsulated CdSe/ZnS QDs were employed as

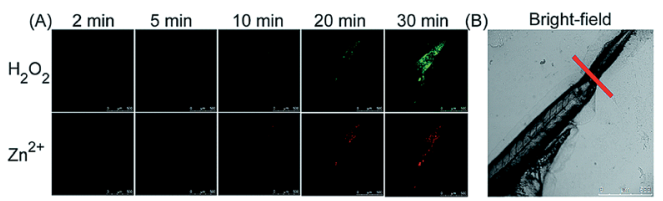

Fig. 33 The simultaneous fluorescence imaging of $\mathrm{H}_{2} \mathrm{O}_{2}$ and $\mathrm{Zn}^{2+}$ in zebrafish upon local injury of the tail fin. (A) The fluorescence images of 68-1 (50 mM, green channel) and $68-2$ (50 mM, red channel). (B) The bright-field image (the red line indicates a cut injury). Adapted with permission from ref. 102b. Copyright 2016 Royal Society of Chemistry. the inner reference. The $\mathrm{pH}$ responsive probe, fluorescein isothiocyanate (FITC) and $\mathrm{O}_{2}{ }^{--}$selective probe, hydroethidine (HE) were grafted onto the surface of the nanocomposites, respectively. The mitochondrion-targeted ratiometric probe was obtained after further TPP modification. With this probe, they revealed that $\mathrm{O}_{2}{ }^{\cdot-}$ could enter into the mitochondria and elevate the $\mathrm{O}_{2}{ }^{\cdot-}$ amount as well as $\mathrm{pH}$, while mitochondrial acidification could cause the $\mathrm{O}_{2}{ }^{\cdot-}$ burst. This probe offered an admirable candidate for investigating the relationships between mitochondrial $\mathrm{O}_{2}{ }^{--}$and $\mathrm{pH}$.

\subsection{Simultaneous imaging of bioactive species in different organelles}

Apart from using multi-analyte responsive molecular or nanoprobes for imaging bioactive species in organelles, simultaneous visualization of bioactive species in different organelles is another important task, since it can facilitate the investigation of bioactive species' fluctuations in different subcellular structures. Visualization of bioactive species in different organelles may offer more opportunities for tracing the "crosstalk" between different organelles and further advance our understanding of diverse physiological and pathological processes (Fig. 35).

Our group demonstrated the simultaneous visualization of $\mathrm{H}_{2} \mathrm{O}_{2}$ fluctuations in the mitochondria and ER with two molecular probes (71-1, MI- $\mathrm{H}_{2} \mathrm{O}_{2}$, mitochondrion-targeted $\mathrm{H}_{2} \mathrm{O}_{2}$ probe, LOD $=80 \mathrm{nM}$ and 71-2, ER- $\mathrm{H}_{2} \mathrm{O}_{2}$, ER targeted $\mathrm{H}_{2} \mathrm{O}_{2}$ 


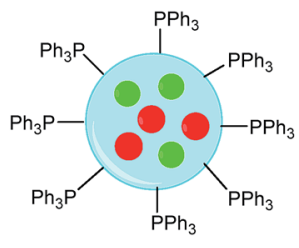

69

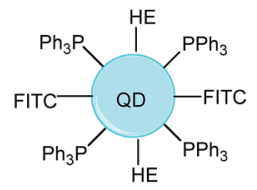

70
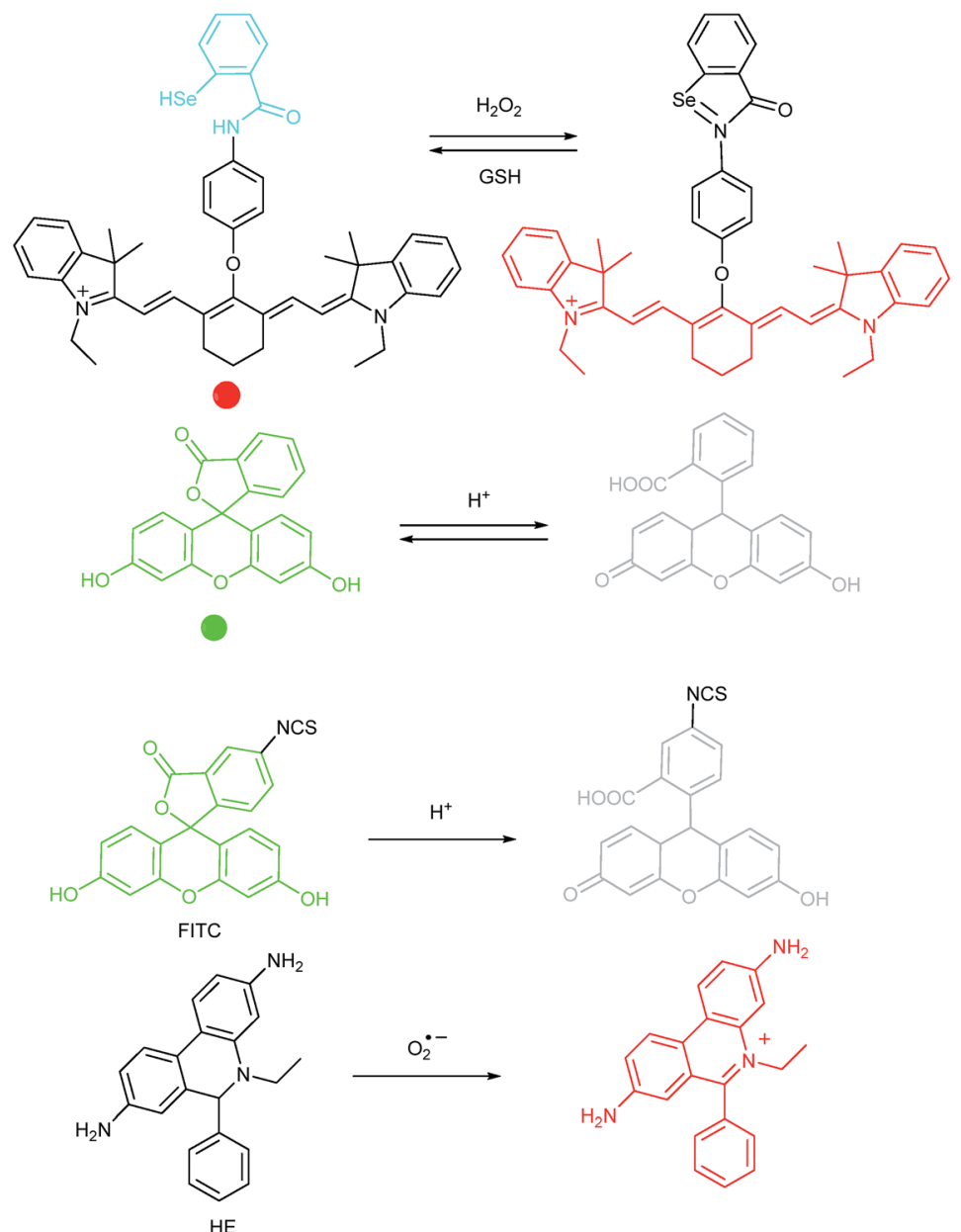

$\mathrm{HE}$

Fig. 34 Nanoprobes for simultaneous imaging of bioactive species in mitochondria.

probe, $\mathrm{LOD}=120 \mathrm{nM}){ }^{30 b} \mathbf{7 1 - 1}$ and $\mathbf{7 1 - 2}$ could accumulate in mitochondria and lysosomes, respectively. Under different stimulations, the $\mathrm{H}_{2} \mathrm{O}_{2}$ concentration in the two subcellular structures could be easily tracked owing to the sensitivity and specificity of the probes, which offered the possibility of realtime discriminating the subcellular $\mathrm{H}_{2} \mathrm{O}_{2}$ fluctuation (Fig. 36). Interestingly, delayed $\mathrm{H}_{2} \mathrm{O}_{2}$ rise effects between the ER and mitochondria during different apoptosis pathways were observed for the first time; this work offered a new method for investigating the "organelle crosstalk" during different processes.

\subsection{Challenges for multi-parameter imaging}

Simultaneous or sequential imaging of the fluctuations of bioactive species in one or different organelles holds great

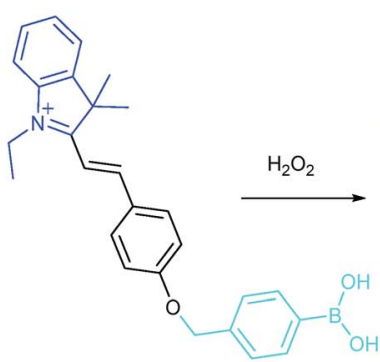

$71-1\left(\mathrm{MI}-\mathrm{H}_{2} \mathrm{O}_{2}\right)$

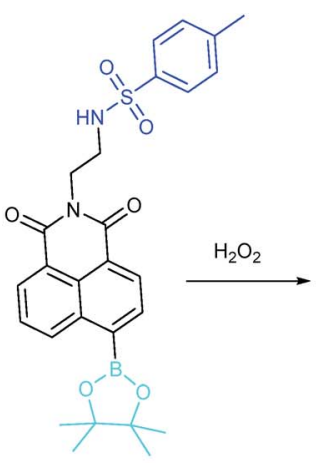

$71-2\left(\mathrm{ER}-\mathrm{H}_{2} \mathrm{O}_{2}\right)$

Fig. 35 Structures and the responses of probes 71-1 and 71-2. 


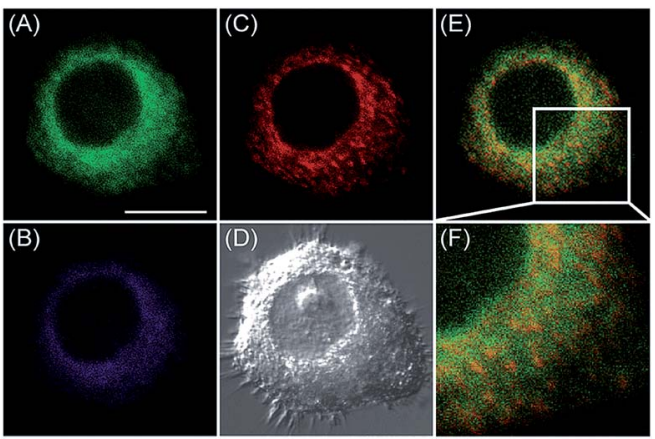

Fig. 36 CLSM images of HepG2 cells stained simultaneously with 71-1 and 71-2 (10 mM). 71-2 was excited at $405 \mathrm{~nm}$, and collected at 500$620 \mathrm{~nm}$ for the green channel (image A) and at 430-470 nm for the blue channel (image B). 71-1 was excited at $543 \mathrm{~nm}$, and collected at 550-600 $\mathrm{nm}$ for the red channel (image C). (D) The bright-field image. (E) The overlay of (A) and (C). (F) The enlarged image from the square marked in image (E). Adapted with permission from ref. 30b. Copyright 2016 Royal Society of Chemistry.

promise for clarifying their upstream and downstream relationships, which will further deepen our understanding of the biological functions of these species and facilitate related bioanalysis. However, construction of molecular probes with two or more responsive moieties is complicated. On the other hand, organelle-targeted nanoprobes usually suffer from inferior targeting efficiency because nanostructures are less permeable during traveling through the complicated intracellular barriers, which makes them usually require longer incubation time during cell imaging. Employment of different molecular tools to image diverse analytes is feasible, and it can easily be extended to investigate communication between different subcellular compartments and open new avenues for bioanalysis. However, probes that have distinct emission bands and can work under single wavelength excitation have not been demonstrated yet.

\section{Conclusion and outlook}

Organelle-targeted RONSS fluorescent probes have aroused increasing interest over the past decade, owing to their potential for better monitoring diverse physiological and pathological activities, and elucidating the molecular mechanisms of different biological events. Based on various fluorophores, recognition groups and targeting ligands, a great number of subcellular localized probes have been elaborately prepared and successfully employed for different applications, including monitoring cancer, diabetes, depression, PDT, drug induced oxidative/nitrative stress, IRI, inflammation and so on. These probes have significantly facilitated our understanding of life science and have undoubtedly become powerful fluorescent tools for bioanalysis. By highlighting the latest and most representative probes, this review summarized current strategies for designing organelle-targeted RONSS probes. The targeting, detection and response mechanisms are carefully discussed, and they will offer valuable references to investigators interested in this area and facilitate the pursuit of better organelle-targeted fluorescent probes. Although the significant superiority of organelle-targeted fluorescent probes over their traditional counterparts has been demonstrated, several obstacles still exist for their practical application. The current challenges as well as the potential future directions are listed below.

\subsection{Solubility}

During living cell imaging, water solubility is a critical parameter for fluorescent probes. The large aromatic structures in fluorescent probes may cause fluorophore aggregation through $\pi-\pi$ stacking, which can not only reduce cellular permeability and subcellular localization, but also quench the fluorescence (aggregation caused quenching effect, ACQ) of fluorescent probes. Organic solvents like DMSO are often used to enhance the solubility of organelle-targeted probes, and this can place extra burden on the cell/tissue samples, and interfere with the subcellular microenvironments. Therefore, development of organelle-targeted probes that have better solubility and can avoid ACQ is highly desired in future work. For this purpose, devising efficient modification strategies and developing watersoluble aggregation induced emission nanoprobes are ideal tactics.

\subsection{Localization capability}

Precisely driving probes into organelles of interest is the prerequisite for subcellular imaging. However, the current available probes suffer from several limitations, including poor targeting efficiency, potential toxic effects and so on. For instance, the widely used mitochondrion targeting group TPP can reduce MMP owing to its cationic feature, and such kind of probe may leak from mitochondria when the MMP is lost. On the other hand, lysosome targeting group morpholine modified probes have an alkalization effect. The high concentration of morpholine decorating the probes can elevate the $\mathrm{pH}$ value of lysosomes, which also has certain toxic effects on the analysed samples. Moreover, highly efficient targeting strategies toward many other important organelles, including the Golgi apparatus, nucleus and lipid droplets, are still rare, which severely limits the determination of critical biological events in these subcellular structures. Genetically encoded probes also suffer from several limitations as discussed above. Therefore, exploration of reliable targeting mechanisms and novel targeting ligands toward diverse subcellular organelles is still urgently required. This aspect relies on the expansion of biological research to identify the intrinsic characteristics and metabolic pathways of diverse organelles. It also relies greatly on chemical synthesis to develop and optimize the structure of targeting ligands. Therefore, collaboration of chemists and biologists is essential for developing highly selective, efficient and non-toxic organelle-targeted ligands, which would further advance chemo-biological investigations.

\subsection{Detection performance}

After reaching the organelles of interest, probes need to specifically and effectively interact with the detected targets for 


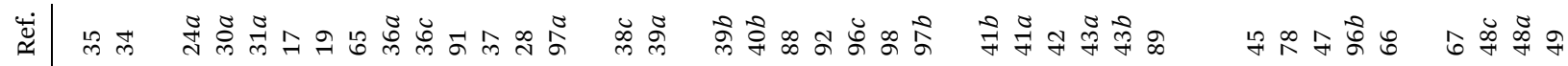

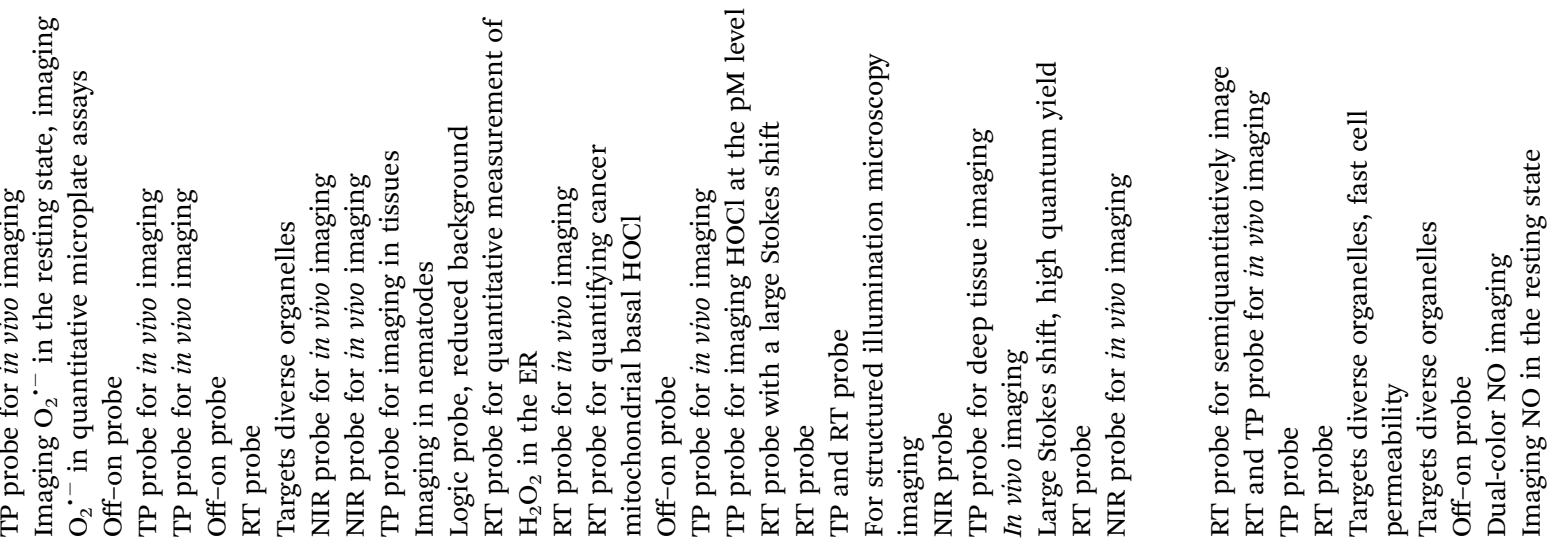

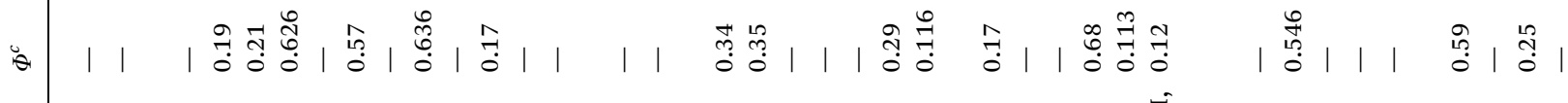

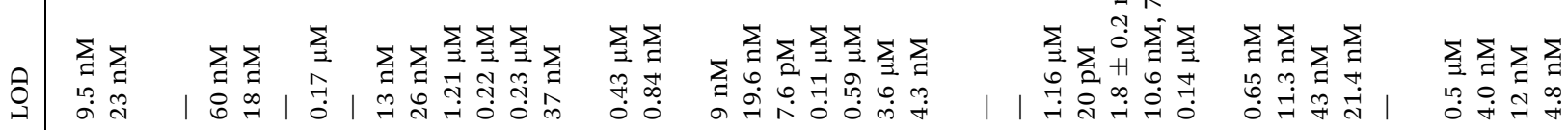

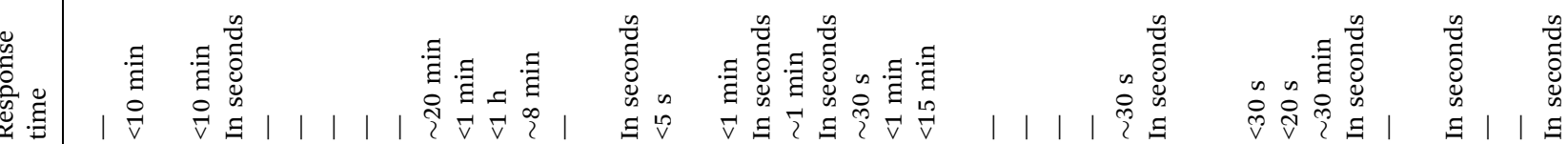

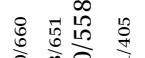

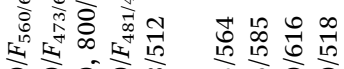

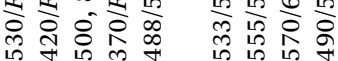

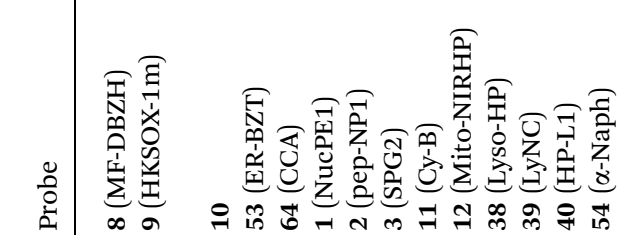

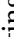
密密 $\stackrel{\circ}{\dot{2}}$

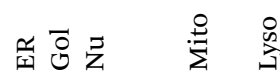
iñ
告定

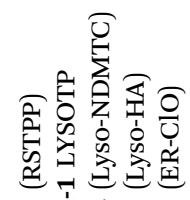

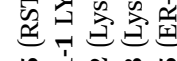
$\frac{0}{2}$ 


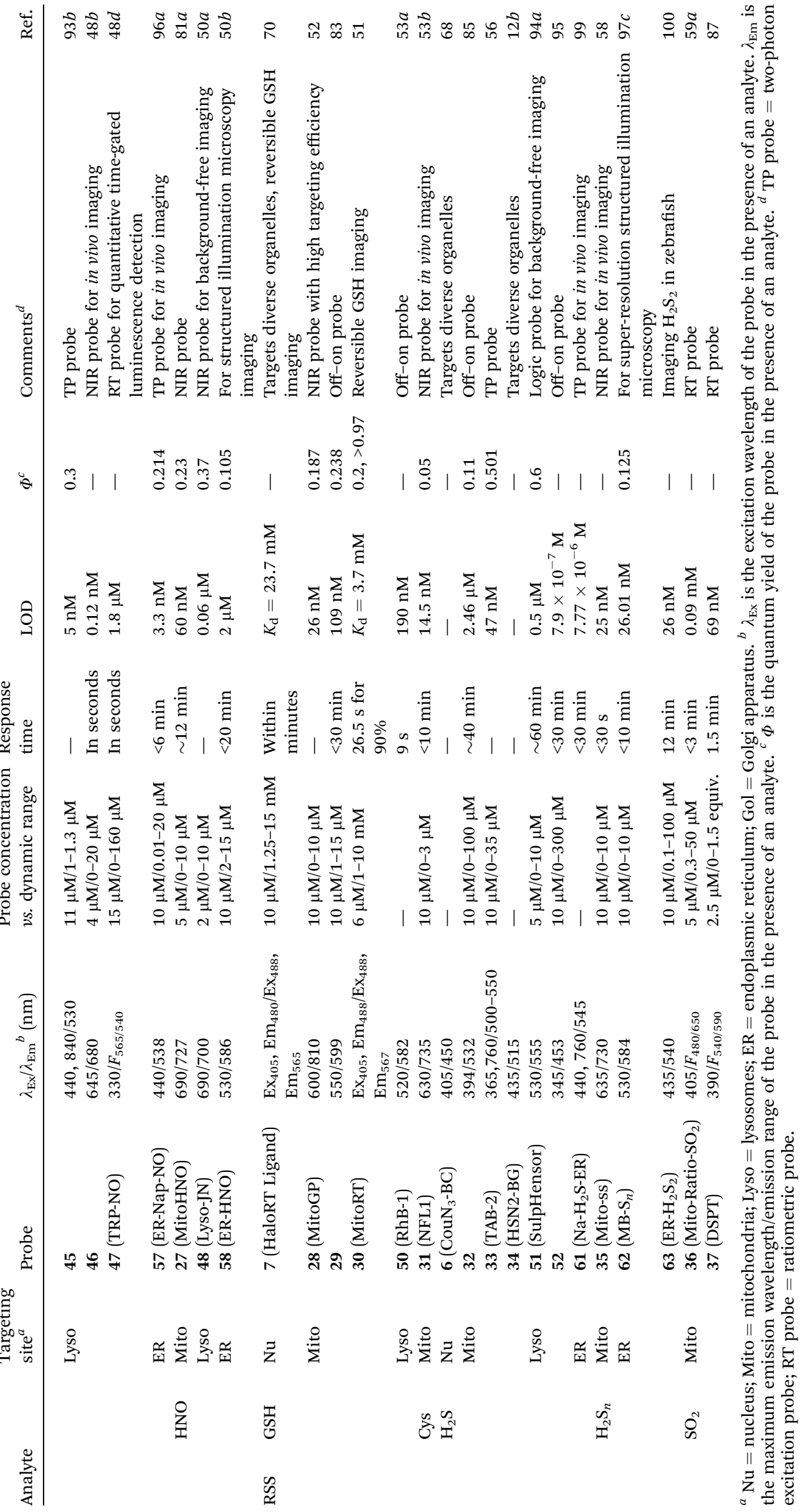


in situ imaging. However, it is well known that bioactive species suffer from ultralow concentration, short life span and environmental sensitivity, while most developed probes failed in tracing the bioactive species in the resting state due to the high LOD, and poor specificity and sensitivity. Therefore, new organelle-targeted probes with higher specificity and sensitivity are highly required. Exploration of new reaction mechanisms, and combination with theoretical calculation as well as high throughput probe screening systems should be promising solutions. In addition, subcellular localized probes with signal amplification have not been investigated, although they should also be study-worthy schemes in future work.

\subsection{Optical performance}

Signal-noise ratio is another critical parameter during subcellular analysis. Many biomolecules, like reduced nicotinamide adenine dinucleotide and some proteins, are emissive under laser irradiation, and can interfere with the resolution and reality of detection information. But the emission wavelengths of most developed organelle-targeted fluorescent probes locate in the visible region, making them unsuitable for backgroundfree/in vivo fluorescent imaging. Therefore, probes with high quantum yields, excellent photostability, long lifetime and strong brightness are attractive. Probes with two or more photon excitation, NIR-I and even NIR-II emissions are also useful for live cell imaging. Development of new fluorophores with higher performances may pave the road to deep penetration, high resolution fluorescence imaging in the near future.

\subsection{Multifunctionality}

Nowadays, theranostic probes have been extensively explored owing to their simplicity and multifunctionality. As reactionbased probes are capable of capturing/adjusting diverse bioactive species, they are promising for controlling diverse disorders caused by RONSS overexpression. However, the therapeutic effects of these probes have rarely been explored. Furthermore, probes with cascade targeting, multiparameter detection and multimodal imaging functions are still rare. Related investigations will tremendously accelerate personalized diagnosis and therapy. Recently, photo-caged probes have drawn great interest for bio-imaging, and they enable us to monitor biomolecules of spatiotemporal interest. However, to the best of our knowledge, such function has not been introduced in organelle-targeted probes.

\subsection{Research models}

Bioactive species participate in almost all the bioactivities and are closely related to diverse major diseases. Research models are indispensable for shedding light on the molecular mechanisms of different biological phenomena. From this review, it can be clearly found that the developed models are still limited. Thus, other models with different important biological activities, including cell immunity, drug resistance, cell communication, cancer metastasis and so on, remain to be further developed and investigated. Besides, communication between bioactive species from different organelles has been demonstrated to be another promising research field, and it should also be explored to further advance our understanding of different biological events.

In summary, considerable research efforts have been devoted to the development of organelle-targeted fluorescent probes during the past decade, with the focus on the preparation of powerful fluorescent tools to enhance the resolution and sensitivity during cell imaging and better explain the molecular mechanisms of diverse biological events. Although research in this field is still in its infancy and many challenges remain to be resolved, it is expected that more organelle-targeted fluorescent probes with high performance will emerge and further advance our understanding in the near future through the conjoint efforts from researchers worldwide.

Finally, a summary of the physiochemical properties of organelle-targeted RONSS probes is provided in the Appendix. However, it is difficult to compare the selectivity/anti-interference capability of probes, even those based on the same detection mechanism, by studying the summary table. We encourage the reader to examine the primary papers and further assess the selectivity during choosing probes for bioanalysis (Table 2).

\section{Appendix}

\section{Conflicts of interest}

The authors declare no competing financial interest.

\section{Acknowledgements}

This work was supported by the National Natural Science Foundation of China (21535004, 91753111, 21874086, and 21775094) and the Key Research and Development Program of Shandong Province (2018YFJH0502).

\section{Notes and references}

1 (a) K. P. Carter, A. M. Young and A. E. Palmer, Chem. Rev., 2014, 114, 4564-4601; (b) H. M. Kim and B. R. Cho, Chem. Rev., 2015, 115, 5014-5055; (c) J. Liu, Z. Cao and Y. Lu, Chem. Rev., 2009, 109, 1948-1998; (d) Z. Guo, S. Park, J. Yoon and I. Shin, Chem. Soc. Rev., 2014, 43, 16-29; (e) V. S. Lin, W. Chen, M. Xian and C. J. Chang, Chem. Soc. Rev., 2015, 44, 4596-4618; (f) L.-Y. Niu, Y.-Z. Chen, H.-R. Zheng, L.-Z. Wu, C.-H. Tung and Q.-Z. Yang, Chem. Soc. Rev., 2015, 44, 61436160; $(g)$ D. Wu, A. C. Sedgwick, T. Gunnlaugsson, E. U. Akkaya, J. Yoon and T. D. James, Chem. Soc. Rev., 2017, 46, 7105-7123; (h) M. C. Heffern, L. M. Matosziuk and T. J. Meade, Chem. Rev., 2013, 114, 4496-4539.

2 (a) J. Chan, S. C. Dodani and C. J. Chang, Nat. Chem., 2012, 4, 973-984; (b) W. Chyan, D. Y. Zhang, S. J. Lippard and R. J. Radford, Proc. Natl. Acad. Sci. U. S. A., 2014, 111, 143148; (c) H. W. Liu, L. Chen, C. Xu, Z. Li, H. Zhang, X. B. Zhang and W. Tan, Chem. Soc. Rev., 2018, 47, 71407180; (d) X. Qian and Z. Xu, Chem. Soc. Rev., 2015, 44, 4487-4493; (e) A. C. Sedgwick, L. Wu, H. H. Han, 
S. D. Bull, X. P. He, T. D. James, J. L. Sessler, B. Z. Tang, H. Tian and J. Yoon, Chem. Soc. Rev., 2018, 47, 8842-8880; (f) Y. Tang, D. Lee, J. Wang, G. Li, J. Yu, W. Lin and J. Yoon, Chem. Soc. Rev., 2015, 44, 5003-5015; (g) Roopa, N. Kumar, V. Bhalla and M. Kumar, Chem. Commun., 2015, 51, 15614-15628.

3 (a) W. Xu, Z. Zeng, J.-H. Jiang, Y.-T. Chang and L. Yuan, Angew. Chem., Int. Ed., 2016, 55, 13658-13699; (b) H. Zhu, J. Fan, J. Du and X. Peng, Acc. Chem. Res., 2016, 49, 21152126; (c) X. Jiao, Y. Li, J. Niu, X. Xie, X. Wang and B. Tang, Anal. Chem., 2018, 90, 533-555; (d) Z. Xu and L. Xu, Chem. Commun., 2016, 52, 1094-1119; (e) R. Zhang, B. Song and J. Yuan, TrAC, Trends Anal. Chem., 2018, 99, 1-33.

4 (a) J. Zielonka, J. Joseph, A. Sikora, M. Hardy, O. Ouari, J. Vasquez-Vivar, G. Cheng, M. Lopez and B. Kalyanaraman, Chem. Rev., 2017, 117, 10043-10120; (b) A. Fernandez and M. Vendrell, Chem. Soc. Rev., 2016, 45, 1182-1196; (c) M. H. Lee, A. Sharma, M. J. Chang, J. Lee, S. Son, J. L. Sessler, C. Kang and J. S. Kim, Chem. Soc. Rev., 2018, 47, 28-52; (d) K. Qiu, Y. Chen, T. W. Rees, L. Ji and H. Chao, Coord. Chem. Rev., 2019, 378, 66-86.

5 (a) T. Kowada, H. Maeda and K. Kikuchi, Chem. Soc. Rev., 2015, 44, 4953-4972; (b) M. H. Lee, J. S. Kim and J. L. Sessler, Chem. Soc. Rev., 2015, 44, 4185-4191.

6 (a) Q. Wan, S. Chen, W. Shi, L. Li and H. Ma, Angew. Chem., Int. Ed., 2014, 53, 10916-10920; (b) K. Zhou, Y. Wang, X. Huang, K. Luby-Phelps, B. D. Sumer and J. Gao, Angew. Chem., Int. Ed., 2011, 50, 6109-6114; (c) M. H. Lee, N. Park, C. Yi, J. H. Han, J. H. Hong, K. P. Kim, D. H. Kang, J. L. Sessler, C. Kang and J. S. Kim, J. Am. Chem. Soc., 2014, 136, 14136-14142; (d) Y. Liu, J. Zhou, L. Wang, X. Hu, X. Liu, M. Liu, Z. Cao, D. Shangguan and W. Tan, J. Am. Chem. Soc., 2016, 138, 12368-12374.

7 (a) N. Jiang, J. Fan, F. Xu, X. Peng, H. Mu, J. Wang and X. Xiong, Angew. Chem., Int. Ed., 2015, 54, 2510-2514; (b) H. Xiao, C. Wu, P. Li and B. Tang, Anal. Chem., 2018, 90, 6081-6088; (c) H. Xiao, P. Li, W. Zhang and B. Tang, Chem. Sci., 2016, 7, 1588-1593; (d) H. Xiao, C. Wu, P. Li, W. Gao, W. Zhang, W. Zhang, L. Tong and B. Tang, Chem. Sci., 2017, 8, 7025-7030.

8 (a) P. Chabosseau, E. Tuncay, G. Meur, E. A. Bellomo, A. Hessels, S. Hughes, P. R. V. Johnson, M. Bugliani, P. Marchetti, B. Turan, A. R. Lyon, M. Merkx and G. A. Rutter, ACS Chem. Biol., 2014, 9, 2111-2120; (b) A. M. Hessels, P. Chabosseau, M. H. Bakker, W. Engelen, G. A. Rutter, K. M. Taylor and M. Merkx, ACS Chem. Biol., 2015, 10, 2126-2134; (c) Y. Han, C. Ding, J. Zhou and Y. Tian, Anal. Chem., 2015, 87, 5333-5339; (d) K. P. Divya, S. Sreejith, P. Ashokkumar, K. Yuzhan, Q. Peng, S. K. Maji, Y. Tong, H. Yu, Y. Zhao, P. Ramamurthy and A. Ajayaghosh, Chem. Sci., 2014, 5, 3469-3474; (e) Y. Chen, Y. Bai, Z. Han, W. He and Z. Guo, Chem. Soc. Rev., 2015, 44, 4517-4546; $(f)$ Y. Matsui, Y. Funato, H. Imamura, H. Miki, S. Mizukami and K. Kikuchi, Chem. Sci., 2017, 8, 8255-8264; $(g)$ G. Zhang, J. J. Gruskos, M. S. Afzal and D. Buccella, Chem. Sci., 2015, 6, 6841-6846; (h) A. Rodriguez-Garcia, J. RojoRuiz, P. Navas-Navarro, F. J. Aulestia, S. Gallego-Sandin,
J. Garcia-Sancho and M. T. Alonso, Proc. Natl. Acad. Sci. U. S. A., 2014, 111, 2584-2589.

9 (a) Z. Yang, Y. He, J.-H. Lee, N. Park, M. Suh, W. S. Chae, J. Cao, X. Peng, H. Jung, C. Kang and J. S. Kim, J. Am. Chem. Soc., 2013, 135, 9181-9185; (b) D. Li, X. Tian, A. Wang, L. Guan, J. Zheng, F. Li, S. Li, H. Zhou, J. Wu and Y. Tian, Chem. Sci., 2016, 7, 2257-2263; (c) M. K. Kuimova, S. W. Botchway, A. W. Parker, M. Balaz, H. A. Collins, H. L. Anderson, K. Suhling and P. R. Ogilby, Nat. Chem., 2009, 1, 69-73.

10 (a) L. M. Hyman and K. J. Franz, Coord. Chem. Rev., 2012, 256, 2333-2356; (b) A. J. Shuhendler, K. Pu, L. Cui, J. P. Uetrecht and J. Rao, Nat. Biotechnol., 2014, 32, 373380; (c) H.-S. Wang, TrAC, Trends Anal. Chem., 2016, 85, 181-202.

11 (a) X. Chen, F. Wang, J. Y. Hyun, T. Wei, J. Qiang, X. Ren, I. Shin and J. Yoon, Chem. Soc. Rev., 2016, 45, 2976-3016; (b) X. Jiang, L. Wang, S. L. Carroll, J. Chen, M. C. Wang and J. Wang, Antioxid. Redox Signaling, 2018, 29, 518-540.

12 (a) X. Yang, Y. Zhou, X. Zhang, S. Yang, Y. Chen, J. Guo, X. Li, Z. Qing and R. Yang, Chem. Commun., 2016, 52, 10289-10292; (b) L. A. Montoya and M. D. Pluth, Anal. Chem., 2016, 88, 5769-5774; (c) H. D. Li, Q. C. Yao, J. L. Fan, N. Jiang, J. Y. Wang, J. Xia and X. J. Peng, Chem. Commun., 2015, 51, 16225-16228; (d) V. S. Lin, A. R. Lippert and C. J. Chang, Proc. Natl. Acad. Sci. U. S. A., 2013, 110, 7131-7135; (e) B. Tang, L. Yin, X. Wang, Z. Chen, L. Tong and K. Xu, Chem. Commun., 2009, 52935295; (f) Q. Gao, W. Zhang, B. Song, R. Zhang, W. Guo and J. Yuan, Anal. Chem., 2017, 89, 4517-4524; (g) F. Yang, H. Gao, S. S. Li, R. B. An, X. Y. Sun, B. Kang, J. J. Xu and H. Y. Chen, Chem. Sci., 2018, 9, 5556-5563.

13 (a) K.-Y. Tan, C.-Y. Li, Y.-F. Li, J. Fei, B. Yang, Y.-J. Fu and F. Li, Anal. Chem., 2017, 89, 1749-1756; (b) L. Wang, L. Yuan, X. Zeng, J. Peng, Y. Ni, J. C. Er, W. Xu, B. K. Agrawalla, D. Su, B. Kim and Y.-T. Chang, Angew. Chem., Int. Ed., 2016, 55, 1773-1776; (c) J. Deng, K. Wang, M. Wang, P. Yu and L. Mao, J. Am. Chem. Soc., 2017, 139, 5877-5882.

14 (a) Y. Tang, X. Kong, A. Xu, B. Dong and W. Lin, Angew. Chem., Int. Ed., 2016, 55, 3356-3359; (b) X. Xie, F. Tang, X. Shangguan, S. Che, J. Niu, Y. Xiao, X. Wang and B. Tang, Chem. Commun., 2017, 53, 6520-6523; (c) T. F. Brewer and C. J. Chang, J. Am. Chem. Soc., 2015, 137, 10886-10889; (d) A. Roth, H. Li, C. Anorma and J. Chan, J. Am. Chem. Soc., 2015, 137, 10890-10893.

15 (a) V. S. Harrison, C. E. Carney, K. W. MacRenaris, E. A. Waters and T. J. Meade, J. Am. Chem. Soc., 2015, 137, 9108-9116; (b) H. Li, G. Parigi, C. Luchinat and T. J. Meade, J. Am. Chem. Soc., 2019, 141, 6224-6233.

16 (a) A. Nakamura, K. Takigawa, Y. Kurishita, K. Kuwata, M. Ishida, Y. Shimoda, I. Hamachi and S. Tsukiji, Chem. Commun., 2014, 50, 6149-6152; (b) Y. Cheng, C. Sun, X. Ou, B. Liu, X. Lou and F. Xia, Chem. Sci., 2017, 8, 45714578.

17 B. C. Dickinson, Y. Tang, Z. Chang and C. J. Chang, Chem. Biol., 2011, 18, 943-948. 
18 G. Lukinavicius, C. Blaukopf, E. Pershagen, A. Schena, L. Reymond, E. Derivery, M. Gonzalez-Gaitan, E. D'Este, S. W. Hell, D. W. Gerlich and K. Johnsson, Nat. Commun., 2015, 6, 8497.

19 Y. Wen, K. Liu, H. Yang, Y. Li, H. Lan, Y. Liu, X. Zhang and T. Yi, Anal. Chem., 2014, 86, 9970-9976.

20 (a) K. Henze and W. Martin, Nature, 2003, 426, 127-128; (b) M. T. Lin and M. F. Beal, Nature, 2006, 443, 787-795; (c) K. Krumova, L. E. Greene and G. Cosa, J. Am. Chem. Soc., 2013, 135, 17135-17143; (d) E. K. Lei and S. O. Kelley, J. Am. Chem. Soc., 2017, 139, 9455-9458; (e) C. W. T. Leung, Y. Hong, S. Chen, E. Zhao, J. W. Y. Lam and B. Z. Tang, J. Am. Chem. Soc., 2013, 135, 62-65.

21 (a) B. C. Dickinson and C. J. Chang, J. Am. Chem. Soc., 2008, 130, 9638-9639; (b) J. Qian and B. Z. Tang, Chem, 2017, 3, 56-91.

22 (a) L. V. Johnson, M. L. Walsh and L. B. Chen, Proc. Natl. Acad. Sci. U. S. A., 1980, 77, 990-994; (b) K. L. Horton, K. M. Stewart, S. B. Fonseca, Q. Guo and S. O. Kelley, Chem. Biol., 2008, 15, 375-382; (c) L. B. Chen, Annu. Rev. Cell Biol., 1988, 4, 155-181.

23 (a) A. R. Sarkar, C. H. Heo, H. W. Lee, K. H. Park, Y. H. Suh and H. M. Kim, Anal. Chem., 2014, 86, 5638-5641; (b) Y. Wen, K. Liu, H. Yang, Y. Liu, L. Chen, Z. Liu, C. Huang and T. Yi, Anal. Chem., 2015, 87, 10579-10584; (c) Z. Xue, R. Zhu, S. Wang, J. Li, J. Han, J. Liu and S. Han, Anal. Chem., 2018, 90, 2954-2962; (d) Y. Chen, C. Zhu, J. Cen, Y. Bai, W. He and Z. Guo, Chem. Sci., 2015, 6, 3187-3194; (e) T. B. Ren, Q. L. Zhang, D. Su, X. X. Zhang, L. Yuan and X. B. Zhang, Chem. Sci., 2018, 9, 5461-5466.

24 (a) F. Si, Y. Liu, K. Yan and W. Zhong, Chem. Commun., 2015, 51, 7931-7934; (b) H. Wang, Z. Gao, X. Liu, P. Agarwal, S. Zhao, D. W. Conroy, G. Ji, J. Yu, C. P. Jaroniec, Z. Liu, X. Lu, X. Li and X. He, Nat. Commun., 2018, 9, 562-577; (c) L. D. Field, J. B. Delehanty, Y. Chen and I. L. Medintz, Acc. Chem. Res., 2015, 48, 1380-1390; (d) S. R. Jean, M. Ahmed, E. K. Lei, S. P. Wisnovsky and S. O. Kelley, Acc. Chem. Res., 2016, 49, 1893-1902.

25 (a) C. Wang, T. Zhao, Y. Li, G. Huang, M. A. White and J. Gao, Adv. Drug Delivery Rev., 2017, 113, 87-96; (b) H. Lan, F. Xue, Z. Liu, L. Chen, C. Huang and T. Yi, Adv. Opt. Mater., 2016, 4, 1367-1372; (c) Z. Wu and X. Tang, Anal. Chem., 2015, 87, 8613-8617.

26 (a) X. Zhang, B. Wang, C. Wang, L. Chen and Y. Xiao, Anal. Chem., 2015, 87, 8292-8300; (b) Z. Wu, D. Liang and X. Tang, Anal. Chem., 2016, 88, 9213-9218; (c) W. Zhu, X. Chai, B. Wang, Y. Zou, T. Wang, Q. Meng and Q. Wu, Chem. Commun., 2015, 51, 9608-9611; (d) Y. Cai, C. Gui, K. Samedov, H. Su, X. Gu, S. Li, W. Luo, H. H. Y. Sung, J. W. Y. Lam, R. T. K. Kwok, I. D. Williams, A. Qin and B. Z. Tang, Chem. Sci., 2017, 8, 7593-7603; (e) R. Duncan, J. Kopecek and J. B. Lloyd, Biochem. Soc. Trans., 1984, 12, 913-915.

27 (a) G. Niu, P. Zhang, W. Liu, M. Wang, H. Zhang, J. Wu, L. Zhang and P. Wang, Anal. Chem., 2017, 89, 1922-1929; (b) R. A. Firestone, J. M. Pisano and R. J. Bonney, J. Med. Chem., 1979, 22, 1130-1133.

28 J. Liu, J. Ren, X. Bao, W. Gao, C. Wu and Y. Zhao, Anal. Chem., 2016, 88, 5865-5870.

29 (a) J. M. Meinig, L. Fu and B. R. Peterson, Angew. Chem., Int. Ed., 2015, 54, 9696-9699; (b) M. Terasaki, J. Song, J. R. Wong, M. J. Weiss and L. B. Chen, Cell, 1984, 38, 101-108.

30 (a) H. Xiao, X. Liu, C. Wu, Y. Wu, P. Li, X. Guo and B. Tang, Biosens. Bioelectron., 2017, 91, 449-455; (b) H. Xiao, P. Li, X. Hu, X. Shi, W. Zhang and B. Tang, Chem. Sci., 2016, 7, 6153-6159; (c) P. Li, X. Shi, H. Xiao, Q. Ding, X. Bai, C. Wu, W. Zhang and B. Tang, Analyst, 2018, 144, 191-196. 31 (a) W. Zhang, J. Zhang, P. Li, J. Liu, D. Su and B. Tang, Chem. Sci., 2018, 10, 879-883; (b) R. S. Li, P. F. Gao, H. Z. Zhang, L. L. Zheng, C. M. Li, J. Wang, Y. F. Li, F. Liu, N. Li and C. Z. Huang, Chem. Sci., 2017, 8, 68296835; (c) P. Li, X. Guo, X. Bai, X. Wang, Q. Ding, W. Zhang, W. Zhang and B. Tang, Anal. Chem., 2019, 91, 3382-3388; (d) T. Hirayama, M. Inden, H. Tsuboi, M. Niwa, Y. Uchida, Y. Naka, I. Hozumi and H. Nagasawa, Chem. Sci., 2019, 10, 1514-1521.

32 A. Keppler, S. Gendreizig, T. Gronemeyer, H. Pick, H. Vogel and K. Johnsson, Nat. Biotechnol., 2003, 21, 86-89.

33 G. V. Los, L. P. Encell, M. G. McDougall, D. D. Hartzell, N. Karassina, C. Zimprich, M. G. Wood, R. Learish, R. F. Ohana, M. Urh, D. Simpson, J. Mendez, K. Zimmerman, P. Otto, G. Vidugiris, J. Zhu, A. Darzins, D. H. Klaubert, R. F. Bulleit and K. V. Wood, ACS Chem. Biol., 2008, 3, 373-382.

34 J. J. Hu, N.-K. Wong, S. Ye, X. Chen, M.-Y. Lu, A. Q. Zhao, Y. Guo, A. C.-H. Ma, A. Y.-H. Leung, J. Shen and D. Yang, J. Am. Chem. Soc., 2015, 137, 6837-6843.

35 P. Li, W. Zhang, K. Li, X. Liu, H. Xiao, W. Zhang and B. Tang, Anal. Chem., 2013, 85, 9877-9881.

36 (a) F. Xu, H. Li, Q. Yao, J. Fan, J. Wang and X. Peng, J. Mater. Chem. B, 2016, 4, 7363-7367; (b) G. Masanta, C. H. Heo, C. S. Lim, S. K. Bae, B. R. Cho and H. M. Kim, Chem. Commun., 2012, 48, 3518-3520; (c) X. Xie, X. Yang, T. Wu, Y. Li, M. Li, Q. Tan, X. Wang and B. Tang, Anal. Chem., 2016, 88, 8019-8025.

37 S. I. Reja, M. Gupta, N. Gupta, V. Bhalla, P. Ohri, G. Kaur and M. Kumar, Chem. Commun., 2017, 53, 3701-3704.

38 (a) Q. Xu, C. H. Heo, J. A. Kim, H. S. Lee, Y. Hu, D. Kim, K. M. K. Swamy, G. Kim, S.-J. Nam, H. M. Kim and J. Yoon, Anal. Chem., 2016, 88, 6615-6620; (b) G. Cheng, J. Fan, W. Sun, K. Sui, X. Jin, J. Wang and X. Peng, Analyst, 2013, 138, 6091-6096; (c) H. Xiao, J. Li, J. Zhao, G. Yin, Y. Quan, J. Wang and R. Wang, J. Mater. Chem. B, 2015, 3, 1633-1638.

39 (a) J.-T. Hou, K. Li, J. Yang, K.-K. Yu, Y.-X. Liao, Y.-Z. Ran, Y.-H. Liu, X.-D. Zhou and X.-Q. Yu, Chem. Commun., 2015, 51, 6781-6784; (b) J. Zhou, L. Li, W. Shi, X. Gao, X. Li and H. Ma, Chem. Sci., 2015, 6, 4884-4888.

40 (a) B. Zhang, X. Yang, R. Zhang, Y. Liu, X. Ren, M. Xian, Y. Ye and Y. Zhao, Anal. Chem., 2017, 89, 10384-10390; (b) L. Yuan, L. Wang, B. K. Agrawalla, S.-J. Park, H. Zhu, 
B. Sivaraman, J. Peng, Q.-H. Xu and Y.-T. Chang, J. Am. Chem. Soc., 2015, 137, 5930-5938; (c) F. Tian, Y. Jia, Y. Zhang, W. Song, G. Zhao, Z. Qu, C. Li, Y. Chen and P. Li, Biosens. Bioelectron., 2016, 86, 68-74; (d) Z. Qu, J. Ding, M. Zhao and P. Li, J. Photochem. Photobiol., A, 2015, 299, 1-8.

41 (a) H.-W. Liu, S. Xu, P. Wang, X.-X. Hu, J. Zhang, L. Yuan, X.-B. Zhang and W. Tan, Chem. Commun., 2016, 52, 12330-12333; (b) S. Kim, T. Tachikawa, M. Fujitsuka and T. Majima, J. Am. Chem. Soc., 2014, 136, 11707-11715.

42 F. Liu, J. Du, D. Song, M. Xu and G. Sun, Chem. Commun., 2016, 52, 4636-4639.

43 (a) X. Liu, A. Zheng, D. Luan, X. Wang, F. Kong, L. Tong, K. Xu and B. Tang, Anal. Chem., 2017, 89, 1787-1792; (b) H. Huang and Y. Tian, Chem. Commun., 2018, 54, 1219812201.

44 H. Li, X. Li, X. Wu, W. Shi and H. Ma, Anal. Chem., 2017, 89, 5519-5525.

45 X. Jia, Q. Chen, Y. Yang, Y. Tang, R. Wang, Y. Xu, W. Zhu and X. Qian, J. Am. Chem. Soc., 2016, 138, 10778-10781.

46 A. C. Sedgwick, H.-H. Han, J. E. Gardiner, S. D. Bull, X.-P. He and T. D. James, Chem. Commun., 2017, 53, 12822-12825.

47 D. Cheng, W. Xu, L. Yuan and X. Zhang, Anal. Chem., 2017, 89, 7693-7700.

48 (a) Y.-Q. Sun, J. Liu, H. Zhang, Y. Huo, X. Lv, Y. Shi and W. Guo, J. Am. Chem. Soc., 2014, 136, 12520-12523; (b) Y. Huo, J. Miao, L. Han, Y. Li, Z. Li, Y. Shi and W. Guo, Chem. Sci., 2017, 8, 6857-6864; (c) H. Yu, X. Zhang, Y. Xiao, W. Zou, L. Wang and L. Jin, Anal. Chem., 2013, 85, 7076-7084; (d) Z. Dai, L. Tian, B. Song, X. Liu and J. Yuan, Chem. Sci., 2017, 8, 1969-1976.

49 J. Miao, Y. Huo, X. Lv, Z. Li, H. Cao, H. Shi, Y. Shi and W. Guo, Biomaterials, 2016, 78, 11-19.

50 (a) X. Jing, F. Yu and L. Chen, Chem. Commun., 2014, 50, 14253-14256; (b) F. Ali, S. Sreedharan, A. H. Ashoka, H. K. Saeed, C. G. W. Smythe, J. A. Thomas and A. Das, Anal. Chem., 2017, 89, 12087-12093.

51 J. Chen, X. Jiang, C. Zhang, K. R. MacKenzie, F. Stossi, T. Palzkill, M. C. Wang and J. Wang, ACS Sens., 2017, 2, 1257-1261.

52 S.-Y. Lim, K.-H. Hong, D. I. Kim, H. Kwon and H. J. Kim, J. Am. Chem. Soc., 2014, 136, 7018-7025.

53 (a) R. Huang, B.-B. Wang, X.-M. Si-Tu, T. Gao, F.-F. Wang, H. He, X.-Y. Fan, F.-L. Jiang and Y. Liu, Chem. Commun., 2016, 52, 11579-11582; (b) C. Han, H. Yang, M. Chen, Q. Su, W. Feng and F. Li, ACS Appl. Mater. Interfaces, 2015, 7, 27968-27975; (c) C.-Y. Kim, H.-J. Kang, S.-J. Chung, H.-K. Kim, S.-Y. Na and H.-J. Kim, Anal. Chem., 2016, 88, 7178-7182.

54 X. Zhang, H. Tan, Y. Yan, Y. Hang, F. Yu, X. Qu and J. Hua, J. Mater. Chem. B, 2017, 5, 2172-2180.

55 L. Zhou, D. Lu, Q. Wang, S. Liu, Q. Lin and H. Sun, Biosens. Bioelectron., 2017, 91, 699-705.

56 J. Liu, X. Guo, R. Hu, X. Liu, S. Wang, S. Li, Y. Li and G. Yang, Anal. Chem., 2016, 88, 1052-1057.

57 X. Feng, T. Zhang, J.-T. Liu, J.-Y. Miao and B.-X. Zhao, Chem. Commun., 2016, 52, 3131-3134.
58 M. Gao, F. Yu, H. Chen and L. Chen, Anal. Chem., 2015, 87, 3631-3638.

59 (a) W. Xu, C. L. Teoh, J. Peng, D. Su, L. Yuan and Y.-T. Chang, Biomaterials, 2015, 56, 1-9; (b) Y. Liu, K. Li, M.-Y. Wu, Y.-H. Liu, Y.-M. Xie and X.-Q. Yu, Chem. Commun., 2015, 51, 10236-10239.

60 W. Pan, Y. Li, M. Wang, H. Yang, N. Li and B. Tang, Chem. Commun., 2016, 52, 4569-4572.

61 M. Luan, N. Li, W. Pan, L. Yang, Z. Yu and B. Tang, Chem. Commun., 2017, 53, 356-359.

62 W. Zhang, P. Li, F. Yang, X. Hu, C. Sun, W. Zhang, D. Chen and B. Tang, J. Am. Chem. Soc., 2013, 135, 14956-14959.

63 X. Li, S. Zhang, J. Cao, N. Xie, T. Liu, B. Yang, Q. He and Y. Hu, Chem. Commun., 2013, 49, 8656-8658.

64 (a) J. Seo, S. Kim and S. Y. Park, J. Am. Chem. Soc., 2004, 126, 11154-11155; (b) J. Zhao, S. Ji, Y. Chen, H. Guo and P. Yang, Phys. Chem. Chem. Phys., 2012, 14, 8803-8817.

65 D. Srikun, A. E. Albers, C. I. Nam, A. T. Iavarone and C. J. Chang, J. Am. Chem. Soc., 2010, 132, 4455-4465.

66 J. Wang, Y. Zhao, C. Wang, Q. Zhu, Z. Du, A. Hu and Y. Yang, PLoS One, 2015, 10, e0123986.

67 C. Wang, X. B. Song, Z. Han, X. Y. Li, Y. P. Xu and Y. Xiao, ACS Chem. Biol., 2016, 11, 2033-2040.

68 J. Chen, M. Zhao, X. Jiang, A. Sizovs, M. C. Wang, C. R. Provost, J. Huang and J. Wang, Analyst, 2016, 141, 1209-1213.

69 A. Gautier, A. Juillerat, C. Heinis, I. R. Correa Jr, M. Kindermann, F. Beaufils and K. Johnsson, Chem. Biol., 2008, 15, 128-136.

70 X. Jiang, C. Zhang, J. Chen, S. Choi, Y. Zhou, M. Zhao, X. Song, X. Chen, M. Maletic-Savatic, T. Palzkill, D. Moore, M. C. Wang and J. Wang, Antioxid. Redox Signaling, 2019, 30, 1900-1910.

71 (a) L. Pan, Q. He, J. Liu, Y. Chen, M. Ma, L. Zhang and J. Shi, J. Am. Chem. Soc., 2012, 134, 5722-5725; (b) Z. Yu, W. Pan, N. Li and B. Tang, Chem. Sci., 2016, 7, 4237-4244.

72 A. T. Hoye, J. E. Davoren, P. Wipf, M. P. Fink and V. E. Kagan, Acc. Chem. Res., 2008, 41, 87-97.

73 (a) J. Qiao, Z. Liu, Y. Tian, M. Wu and Z. Niu, Chem. Commun., 2015, 51, 3641-3644; (b) F. Du, Y. Min, F. Zeng, C. Yu and S. Wu, Small, 2014, 10, 964-972.

74 Q. Hu, C. Qin, L. Huang, H. Wang, Q. Liu and L. Zeng, Dyes Pigm., 2018, 149, 253-260.

75 (a) J.-T. Hou, M.-Y. Wu, K. Li, J. Yang, K.-K. Yu, Y.-M. Xie and X.-Q. Yu, Chem. Commun., 2014, 50, 8640-8643; (b) K. Li, J.-T. Hou, J. Yang and X.-Q. Yu, Chem. Commun., 2017, 53, 5539-5541.

76 K. Xu, D. Luan, X. Wang, B. Hu, X. Liu, F. Kong and B. Tang, Angew. Chem., Int. Ed., 2016, 55, 12751-12754.

77 (a) Q. Hu, M. Gao, G. Feng and B. Liu, Angew. Chem., Int. Ed., 2014, 53, 14225-14229; (b) W. Lv, Z. Zhang, K. Y. Zhang, H. Yang, S. Liu, A. Xu, S. Guo, Q. Zhao and W. Huang, Angew. Chem., Int. Ed., 2016, 55, 9947-9951; (c) C. Gui, E. Zhao, R. T. K. Kwok, A. C. S. Leung, J. W. Y. Lam, M. Jiang, H. Deng, Y. Cai, W. Zhang, H. Su and B. Z. Tang, Chem. Sci., 2017, 8, 1822-1830; (d) H.-W. Liu, X.-X. Hu, K. Li, Y. Liu, Q. Rong, L. Zhu, 
L. Yuan, F.-L. Qu, X.-B. Zhang and W. Tan, Chem. Sci., 2017, 8, 7689-7695; (e) S. Chakrabortty, B. K. Agrawalla, A. Stumper, N. M. Vegi, S. Fischer, C. Reichardt, M. Kogler, B. Dietzek, M. Feuring-Buske, C. Buske, S. Rau and T. Weil, J. Am. Chem. Soc., 2017, 139, 2512-2519.

78 D. Cheng, Y. Pan, L. Wang, Z. Zeng, L. Yuan, X. Zhang and Y.-T. Chang, J. Am. Chem. Soc., 2017, 139, 285-292.

79 B. C. Zhu, M. Zhang, L. Wu, Z. Y. Zhao, C. Y. Liu, Z. K. Wang, Q. X. Duan, Y. W. Wang and P. Jia, Sens. Actuators, B, 2018, 257, 436-441.

80 K. Sunwoo, K. N. Bobba, J.-Y. Lim, T. Park, A. Podder, J. S. Heo, S. G. Lee, S. Bhuniya and J. S. Kim, Chem. Commun., 2017, 53, 1723-1726.

81 (a) X. Gong, X.-F. Yang, Y. Zhong, Y. Chen and Z. Li, Dyes Pigm., 2016, 131, 24-32; (b) M. Ren, B. Deng, K. Zhou, J.-Y. Wang, X. Kong and W. Lin, J. Mater. Chem. B, 2017, 5, 1954-1961.

82 D. P. Li, Z.-Y. Wang, X.-J. Cao, J. Cui, X. Wang, H.-Z. Cui, J.-Y. Miao and B.-X. Zhao, Chem. Commun., 2016, 52, 2760-2763.

83 J. Zhang, X. Bao, J. Zhou, F. Peng, H. Ren, X. Dong and W. Zhao, Biosens. Bioelectron., 2016, 85, 164-170.

84 (a) W. Niu, L. Guo, Y. Li, S. Shuang, C. Dong and M. S. Wong, Anal. Chem., 2016, 88, 1908-1914; (b) K. Yin, F. Yu, W. Zhang and L. Chen, Biosens. Bioelectron., 2015, 74, 156-164.

85 Y. L. Pak, J. Li, K. C. Ko, G. Kim, J. Y. Lee and J. Yoon, Anal. Chem., 2016, 88, 5476-5481.

86 L. Tang, P. He, X. Yan, J. Sun, K. Zhong, S. Hou and Y. Bian, Sens. Actuators, B, 2017, 247, 421-427.

87 W.-L. Wu, H.-L. Ma, M.-F. Huang, J.-Y. Miao and B.-X. Zhao, Sens. Actuators, B, 2017, 241, 239-244.

88 B. Zhu, P. Li, W. Shu, X. Wang, C. Liu, Y. Wang, Z. Wang, Y. Wang and B. Tang, Anal. Chem., 2016, 88, 12532-12538.

89 H. Zhang, J. Liu, C. Liu, P. Yu, M. Sun, X. Yan, J.-P. Guo and W. Guo, Biomaterials, 2017, 133, 60-69.

90 (a) W. Gao, W. Cao, H. Zhang, P. Li, K. Xu and B. Tang, Chem. Commun., 2014, 50, 8117-8120; (b) W. Gao, L. Ji, L. Li, G. Cui, K. Xu, P. Li and B. Tang, Biomaterials, 2012, 33, 3710-3718.

91 M. Ren, B. Deng, J.-Y. Wang, X. Kong, Z.-R. Liu, K. Zhou, L. He and W. Lin, Biosens. Bioelectron., 2016, 79, 237-243.

92 M. Ren, B. Deng, K. Zhou, X. Kong, J.-Y. Wang, G. Xu and W. Lin, J. Mater. Chem. B, 2016, 4, 4739-4745.

93 (a) B. Wang, S. Yu, X. Chai, T. Li, Q. Wu and T. Wang, Chem.-Eur. J., 2016, 22, 5649-5656; (b) H. Yu, Y. Xiao and L. Jin, J. Am. Chem. Soc., 2012, 134, 17486-17489.
94 (a) S. Yang, Y. Qi, C. Liu, Y. Wang, Y. Zhao, L. Wang, J. Li, W. Tan and R. Yang, Anal. Chem., 2014, 86, 7508-7515; (b) J. Fan, Z. Han, Y. Kang and X. Peng, Sci. Rep., 2016, 6, 19562-19569.

95 X. J. Zou, Y. C. Ma, L. E. Guo, W. X. Liu, M. J. Liu, C. G. Zou, Y. Zhou and J. F. Zhang, Chem. Commun., 2014, 50, 1383313836.

96 (a) S. J. Li, D. Y. Zhou, Y. Li, H. W. Liu, P. Wu, J. Ou-Yang, W. L. Jiang and C. Y. Li, ACS Sens., 2018, 3, 2311-2319; (b) L. Wu, Y. Wang, M. Weber, L. Liu, A. C. Sedgwick, S. D. Bull, C. Huang and T. D. James, Chem. Commun., 2018, 54, 9953-9956; (c) J.-T. Hou, H. S. Kim, C. Duan, M. S. Ji, S. Wang, L. Zeng, W. X. Ren and J. S. Kim, Chem. Commun., 2019, 55, 2533-2536.

97 (a) C. Gao, Y. Tian, R. Zhang, J. Jing and X. Zhang, Anal. Chem., 2017, 89, 12945-12950; (b) F. Ali, S. Aute, S. Sreedharan, H. A. Anila, H. K. Saeed, C. G. Smythe, J. A. Thomas and A. Das, Chem. Commun., 2018, 54, 18491852; (c) A. Hoskere, S. Sreedharan, F. Ali, C. G. Smythe, J. A. Thomas and A. Das, Chem. Commun., 2018, 54, 37353738.

98 Y. L. Pak, S. J. Park, G. Song, Y. Yim, H. Kang, H. M. Kim, J. Bouffard and J. Yoon, Anal. Chem., 2018, 90, 12937-12943.

99 Y. Tang, A. Xu, Y. Ma, G. Xu, S. Gao and W. Lin, Sci. Rep., 2017, 7, 12944.

100 H. Zhou, J. Tang, L. Sun, J. Zhang, B. Chen, J. Kan, W. Zhang, J. Zhang and J. Zhou, Sens. Actuators, B, 2019, 278, 64-72.

101 (a) L. Yang, Y. Chen, Z. Yu, W. Pan, H. Wang, N. Li and B. Tang, ACS Appl. Mater. Interfaces, 2017, 9, 27512-27521; (b) Z. Liu, H. Pei, L. Zhang and Y. Tian, ACS Nano, 2018, 12357-12368; (c) L. Yang, N. Li, W. Pan, Z. Yu and B. Tang, Anal. Chem., 2015, 87, 3678-3684; (d) K. Dou, G. Chen, F. Yu, Z. Sun, G. Li, X. Zhao, L. Chen and J. You, J. Mater. Chem. B, 2017, 5, 8389-8398.

102 (a) Y. Zhou, X. Zhang, S. Yang, Y. Li, Z. Qing, J. Zheng, J. Li and R. Yang, Anal. Chem., 2017, 89, 4587-4594; (b) H. Xiao, P. Li, S. Zhang, W. Zhang, W. Zhang and B. Tang, Chem. Commun., 2016, 52, 12741-12744; (c) F. Yu, M. Gao, M. Li and L. Chen, Biomaterials, 2015, 63, 93-101; (d) W. Zhang, X. Wang, P. Li, H. B. Xiao, W. Zhang, H. Wang and B. Tang, Anal. Chem., 2017, 89, 6840-6845.

103 W. Pan, H. Wang, L. Yang, Z. Yu, N. Li and B. Tang, Anal. Chem., 2016, 88, 6743-6748.

104 H. Huang, F. Dong and Y. Tian, Anal. Chem., 2016, 88, 12294-12302. 